\title{
Dozens of compact and high velocity-dispersion, early-type galaxies in Sloan Digital Sky Survey ${ }^{\star}$
}

\author{
Christoph Saulder ${ }^{1,4}$, Remco C. E. van den Bosch ${ }^{2}$, and Steffen Mieske ${ }^{3}$ \\ ${ }^{1}$ European Southern Observatory, Karl-Schwarzschild-Straße 2, 85748 Garching bei München, Germany \\ e-mail: christoph. saulder@equinoxomega. net \\ 2 Max-Planck-Institut für Astronomie, Königstuhl 17, 69117 Heidelberg, Germany \\ 3 European Southern Observatory, Alonso de Córdova 3107, Vitacura, Casilla 19001, Santiago, Chile \\ ${ }^{4}$ Department of Astrophysics, University of Vienna, Türkenschanzstraße 17, 1180 Vienna, Austria
}

Received 5 December 2014 / Accepted 17 March 2015

\section{ABSTRACT}

\begin{abstract}
Context. Passive galaxies at high redshift are much smaller than equally massive early types today. If this size evolution is caused by stochastic merging processes, then a small fraction of the compact galaxies should persist until today. Up to now it has not been possible to systematically identify the existence of such objects in Sloan Digital Sky Survey (SDSS).

Aims. We aim at finding potential survivors of these compact galaxies in SDSS, as targets for more detailed follow-up observations. Methods. From the virial theorem, it is expected that for a given mass, compact galaxies have stellar velocity dispersion higher than the mean owing to their smaller sizes. Therefore velocity dispersion, coupled with size (or mass), is an appropriate method of selecting relics, independent of the stellar population properties. Based on these considerations, we designed a set of criteria the use the distribution of early-type galaxies from SDSS on the $\log _{10}\left(R_{0}\right)-\log _{10}\left(\sigma_{0}\right)$ plane to find the most extreme objects on it. We thus selected compact massive galaxy candidates by restricting them to high velocity dispersions $\sigma_{0}>323.2 \mathrm{~km} \mathrm{~s}^{-1}$ and small sizes $R_{0}<2.18 \mathrm{kpc}$.

Results. We find 76 galaxies at $0.05<z<0.2$, which have properties that are similar to the typical quiescent galaxies at high redshift. We discuss how these galaxies relate to average present-day early-type galaxies. We study how well these galaxies fit on well-known local universe relations of early-type galaxies, such as the fundamental plane, the red sequence, or mass-size relations. As expected from the selection criteria, the candidates are located in an extreme corner of the mass-size plane. However, they do not extend as deeply into the so-called zone of exclusion as some of the red nuggets found at high redshift, since they are a factor 2-3 less massive on a given intrinsic scale size. Several of our candidates are close to the size resolution limit of SDSS, but are not so small that they are classified as point sources. We find that our candidates are systematically offset on a scaling relation compared to the average early-type galaxies, but still within the general range of other early-type galaxies. Furthermore, our candidates are similar to the mass-size range expected for passive evolution of the red nuggets from their high redshift to the present.

Conclusions. The 76 selected candidates form an appropriate set of objects for further follow-up observations. They do not constitute a separate population of peculiar galaxies, but form the extreme tail of a continuous distribution of early-type galaxies. We argue that selecting a high-velocity dispersion is the best way to find analogues of compact high redshift galaxies in the local universe.
\end{abstract}

Key words. galaxies: elliptical and lenticular, cD - surveys - galaxies: fundamental parameters - galaxies: peculiar

\section{Introduction}

Compact massive early-type galaxies are common at high redshifts $(z>1$; Trujillo et al. 2006; van Dokkum et al. 2008). At those epochs, an average $10^{11} M_{\odot}$ passive galaxy has a size of $1 \mathrm{kpc}$, whereas today's early-type galaxies are three to ten times larger (Taylor et al. 2010), indicating that galaxies undergo a significant amount of size evolution (van der Wel et al. 2014a). If a small fraction of those early galaxies, which are also called red nuggets, evolve completely passively, without any mergers, then some of them must have remained compact until today. Those objects would be pristine relics, which would allow direct insight into how these objects formed long ago. It is therefore interesting to find out if any of those systems still remain today.

Several studies have been done to find such objects using the Sloan Digital Sky Survey (SDSS) photometry with varying success. Taylor et al. (2010) found no analogues of the $z \sim 2$

* Tables 2, 3, and Appendices are available in electronic form at http://www. aanda.org early-type galaxies. Similarly, Trujillo et al. (2009) found few objects, but those turned out to be young with ages of 2 Gyr. Damjanov et al. (2009) found nine objects - some of which were old - indicating that some of the relics must exist. Damjanov et al. (2014) found several object in the BOSS survey and measured a space density of $10^{-6}$ galaxies $\mathrm{Mpc}^{-3}$, which is consistent with expectations from semi-analytic models (Quilis \& Trujillo 2013). In contrast to this, there are also claims of detecting high number densities of these compact massive early-type galaxies in cluster environments (Valentinuzzi et al. 2010), and even in the field (Poggianti et al. 2013), that are in tension with the upper limits of the model predictions (Damjanov et al. 2014). The results from Valentinuzzi et al. (2010) have been debated, and various inconsistencies with other works are pointed out in Taylor et al. (2010).

The virial theorem (and its observational projection, the fundamental plane) predicts that these small galaxies must have high-velocity dispersions. This has been directly confirmed with deep spectroscopy of a handful of these objects (van Dokkum et al. 2009; van de Sande et al. 2013; Toft et al. 2012). This 
makes the dispersion a very good discriminator for finding these very dense objects in the local universe because measuring dispersion is much easier at low redshift. The other advantage is that such a selection is independent of uncertainties in photometric stellar masses. The stellar velocity dispersion was used in van den Bosch et al. (2012) as a discriminator and found six compact objects - including NGC 1277 - in the HETMGS survey (van den Bosch et al. 2015). These objects appear to be consistent with being relics, given their size, mass, and velocity dispersion (van den Bosch et al. 2012). In particular, NGC 1277 has a high dynamical mass-to-light ratio (Emsellem 2013; Yildirım et al., submitted). Subsequently, Trujillo et al. (2014) revealed that its photometry is similar to the nuggets and the stellar population has a uniformly old age. The galaxies of van den Bosch et al. (2012) are lenticular, which agrees well with most compact massive high redshift galaxies being discdominated (van der Wel et al. 2011; Chevance et al. 2012).

This begs the question of whether relics of such compact high- $z$ nuggets can be found as high-dispersion galaxies in SDSS. The starting point of our investigation is [BHF2008] 19, which is the galaxy with ID number 19 on the list of the highest dispersion galaxies in SDSS by Bernardi et al. (2008). It is a very compact and massive early-type galaxy with a size of $R_{\mathrm{e}}=2.17 \mathrm{kpc}$ and mass of $10^{11} M_{\odot}$. We refer to this object as b19 in this paper. It was investigated in great detail in Läsker et al. (2013) and it was found that b19 has a high stellar massto-ratio of $\sim 7 M_{\odot} / L_{\odot, \text { i }}$ and probably a bottom-heavy initial mass function $^{1}$. The object is located at a redshift $z=0.1166$ and is considered to be one of the most compact galaxies for its given mass in the local universe.

In this paper, we performed a systematic search in SDSS to find objects similar to b19, so as to have a broader basis for future investigations of compact, high-dispersion, massive early-type galaxies. The other object, NGC 1277, could not be used for this, because it is not in the main SDSS survey ${ }^{2}$.

While this paper is primarily a sample selection for followup observations, we also touch on the following important questions. Is b19 the most extreme (in the sense of mass and compactness) early-type galaxy in the local universe? Do objects like b19 just form the compact-massive tail of the general distribution of elliptical galaxies, or are they outliers known scaling relations for early-type galaxies? In which aspects do b19-like objects differ from other present-day, early-type galaxies, and are they related to red nuggets from the early universe?

We used selection criteria based on size and central velocity dispersion to find potential red nuggets in the local universe. This is different from what was done by other authors, such as Trujillo et al. (2009) and Taylor et al. (2010), who used selection criteria based on size and stellar mass. It is difficult to measure stellar masses without additional follow-up on the SDSS, and the uncertainties are high with at least 0.1 dex statistical and 0.2 dex systematic error for the stellar masses of Blanton \& Roweis (2007), which were used by Trujillo et al. (2009): about 0.1 dex for the stellar masses used by Taylor et al. (2010), which were based on method of Kauffmann et al. (2003) and Salim et al. (2007), and 0.15 dex for the stellar masses of Mendel et al. (2014), which we used in this paper. To avoid these uncertainties

\footnotetext{
1 A large black hole could not be ruled out by the observations, but even if this system has a large black hole, then the dynamical and stellar population models still work better with a bottom heavy initial mass function.

2 NGC 1277 was observed by SDSS as part of a auxiliary Perseus survey.
}

in our sample definition, we selected with more directly measured quantities, such as the physical radii and the central velocity dispersion. The galaxies of van den Bosch et al. (2012) and b19 are usually assumed to be relics of the red nuggets and if this is the case, there might be more galaxies with similar properties, and some of them may have the same origins. Our method is additionally motivated by the fact that at least a subgroup of red nuggets possess high central velocity dispersions (Newman et al. 2010; Bezanson et al. 2013) and that the stellar-to-dynamical mass-to-light ratio decreases slightly over time (van de Sande et al. 2013). We set out to find them and provide a new selection method that is capable of discovering possible remnants of compact massive red galaxies from the early universe that would have been missed in previous investigations that used different selection criteria. Our goal is to define a sample to be used for follow-up observations to determine whether there is a systematic variation in the initial mass function, such as the bottom-heavy initial mass function of b19 (Läsker et al. 2013), and if they host over-massive central black hole such as the one in NGC 1277 (van den Bosch et al. 2012). Furthermore, follow-up observation will also be required to clean the sample from high central velocity dispersion galaxies that are superpositions of two or more galaxies, which is a known issue with this kind of galaxies in SDSS (Bernardi et al. 2008).

In Sect. 2, we describe the basic sample used for this investigation. After it is calibrated as explained in Sect. 3, we discuss the selection of our candidates in Sect. 4 using various cuts, which are defined there. We investigate the global properties of our candidates and their relation to the basic sample in Sect. 5. We discuss our candidates and their relation to other samples of potential red nugget galaxies in Sect. 6. In Sect. 7, we provide a summary and some concluding remarks on our work. We supplement our paper with three appendices that provide updated fundamental-plane coefficients in Appendix A, additional tables of other samples and their cross-matches with our candidates in Appendix C, and an alternative candidate sample using Sersic profiles instead of the de Vaucouleurs profile in Appendix B.

Throughout this paper, we assume a $\Lambda$-CDM cosmology with the following parameters: relative dark energy density $\Omega_{\Lambda}=0.7$, relative matter density $\Omega_{\mathrm{M}}=0.3$, and a Hubble parameter $H_{0}=70 \mathrm{~km} \mathrm{~s}^{-1} \mathrm{Mpc}^{-1}$.

\section{Basic sample}

As the baseline sample of our search for b19 analogues, we made broad use of the Sloan Digital Sky Surveys (SDSS) and especially of its tenth (Ahn et al. 2014) and seventh (Abazajian et al. 2009) data releases (DR10 and DR7). Furthermore, we used GalaxyZoo (Lintott et al. 2008, 2011) for our galaxy classifications, the refits of SDSS DR7 using Sersic profiles done by Simard et al. (2011), and the stellar masses from Mendel et al. (2014), which is itself based on the previous work of Simard et al. (2011). For comparison, we also used the list of $63 \mathrm{com}-$ pact massive galaxies from Taylor et al. (2010), which is based on SDSS DR7 as well as a list of 29 compact massive galaxies from Trujillo et al. (2009), which is based on the NYU ValueAdded Galaxy Catalog (Blanton et al. 2005) and covers a subsample of SDSS.

We selected all galaxies from the SDSS database that fulfil the following criteria, which are summarized in Table 1: spectroscopic data has to be available and redshifts have to be reliably obtained (zWarning-flag set to zero). We required that the measured redshifts lie between 0 and 0.5 . By using spectroscopic data from SDSS, we implicitly introduced the selection criteria 
Table 1. Selection criteria given in the language of the SDSS CAS-job queries.

\begin{tabular}{cc}
\hline \hline Parameter & Condition \\
\hline SpecObj.z & $>0$ \\
SpecObj.z & $<0.5$ \\
SpecObj.zWarning & $=0$ \\
SpecObj.veldisp & $>100$ \\
SpecObj.snMedian & $>10$ \\
SpecObj.class & $=$ "GALAXY" \\
(P.flags_r\& 0x40000) & $=0$ \\
\hline
\end{tabular}

of SDSS spectroscopy on our data, which are a minimum apparent magnitude in the $r$ band of 17.77 mag (Strauss et al. 2002) and a saturation limit, which corresponds to a maximum magnitude of either $13 \mathrm{mag}$ in the $u$ band, $14 \mathrm{mag}$ in the $g, r$, or $i$ band, or 12 mag in the $z$ band. The central velocity dispersion has to be higher than $100 \mathrm{~km} \mathrm{~s}^{-1}$, the signal-to-noise for spectra has to be better than 10 , and the automatic spectral classification has to confirm that the object is a galaxy. To ensure reliable photometric measurements, we required that there are no saturated objects in our sample (P.flags_r not set to SATURATED). As a direct consequence of these requirements, we required that there must be spectroscopic data for every galaxy in our sample. We imposed the target limit for galaxy spectroscopy of SDSS on our sample, which is a minimum Petrosian magnitude in the $r$ band of $17.77 \mathrm{mag}$ (Strauss et al. 2002) and saturation limit of $13 \mathrm{mag}$ in the $u$ band, 14 mag in the $g, r$, or $i$ band, or 12 mag in the $z$ band.

With these criteria, we found 393033 galaxies in SDSS DR10. For these galaxies, we downloaded the SDSS DR10 object ID, the galactic and equatorial coordinates, the redshift, the central velocity dispersion, and the following photometric quantities for the $g, r, i$, and $z$ filters each: the axis-ratios, the de Vaucouleurs radii, the de Vaucouleurs model magnitudes, the galactic extinction, the likelihoods for a de Vaucouleurs profile and for an exponential profile, and the probability of its being an early-type galaxy based on GalaxyZoo.

We used the Sersic fit radii and magnitudes, as well as the Sersic indices from the catalogue by Simard et al. (2011). This catalogue is based on SDSS DR7, and it only provides the SDSS DR7 ID, which differs from the SDSS DR10 object IDs, to identify the galaxies in the catalogue, but no coordinates to do a direct cross-match. We could revert to SDSS DR7, but we prefer to take advantage of the updated photometry of SDSS DR10 (Ahn et al. 2014). We solved this problem by using a complete set of all SDSS DR7 galaxies with object IDs, equatorial coordinates, and redshifts to create a bridge between our data and the catalogue, which allows for direct cross-identification between them.

We used the stellar masses of SDSS galaxies based on the dusty models of the catalogue by Mendel et al. (2014). Their estimates for stellar masses were derived using a stellar population synthesis based on the code of Conroy et al. (2009) with spectral energy distributions based on the SDSS broadband photometry. We cross-matched this catalogue with the one of Simard et al. (2011) and our DR10 sample. Since the Mendel et al. (2014) catalogue has stricter redshift limits than our SDSS DR10 sample, the measured redshifts of the combined sample have to lie between 0.005 and $0.4^{3}$ now. This also removed all galaxies that might be blended with a nearby star (Mendel et al. 2014). We used the SDSS DR7 Object ID (to cross-match the catalogue with the other samples) and the logarithm of the stellar masses derived from the Sersic profiles and the composite profiles of the Mendel et al. (2014) catalogue. After all these cross-matching, we ended up with a sample of 370159 galaxies.

Additional constraints were applied to the data after the calculation of several parameters from the observed values. Galaxies with a velocity dispersion of higher than $420 \mathrm{~km} \mathrm{~s}^{-1}$ were removed from the sample, because these values would be outside the trusted margin of SDSS algorithm for measuring the central velocity dispersion. We checked that dropping this upper dispersion limit would only contribute galaxies with unreasonably high central velocity dispersions. Furthermore, we eliminated all galaxies with an absolute magnitude either brighter than -25 mag or fainter than -15 mag in any of the used filters. A handful of galaxies with physical radii of more than $10^{2.5} \mathrm{kpc}$ were also removed to avoid contamination from incorrectly measured radii.

Furthermore, the selected galaxies must have been identified as an elliptical galaxy with a probability greater than 0.5 based on GalaxyZoo. We carefully investigated the effect of different values of the criterion on our sample and on the candidates we want to find (see Sect. 4). Values higher than 0.5 will remove too many promising candidates from our sample, while for values below 0.5 , the candidates in our sample will be heavily contaminated by galaxies that are superimposed on another galaxy in the line of sight or near neighbours, galaxies close to a very bright foreground star, and star-burst galaxies. We required that the likelihood for a de Vaucouleurs profile is greater than the likelihood for an exponential profile in every filter except the $u$ band, owing to known problems ${ }^{4}$ with this filter. This criterion was necessary because we used parameters, such as the radii and magnitudes obtained by de Vaucouleurs fits in this paper. If the likelihood for another profile is indeed higher, it would result in poorly derived values for our parameters.

In light of our comparison with other samples of compact massive galaxies in the local universe (see Sect. 5 for details), we found that the vast majority of these galaxies in the literature are within our basic sample and therefore best described by a de Vaucouleurs profile. Since we were searching for potential survivors of the red nuggets, we limited our sample to red sequence galaxies. We did this by removing all galaxies bluer than the lower $3 \sigma$ limit of the red sequence fit performed in Saulder et al. (2013). After this filtering, there were 233833 galaxies between a redshift of 0.005 and 0.4 left (about $59.5 \%$ of the first selection and $63 \%$ of the cross-matched sample). Those form the basic sample that were used for the further analysis in this paper.

\section{Method}

One has to carefully calibrate the parameters obtained from the SDSS database and the refits done by Simard et al. (2011) before using them to classify and characterize the galaxies. The following quantities are calculated for all sources in every band used and for every set of fit parameters.

\footnotetext{
3 The reduced upper redshift limit is no concern to us, since we do not expect to detect any intrinsically small galaxies at redshifts higher than 0.2 anyway.

4 See: https://www.sdss3.org/dr10/imaging/caveats.php\# usky.
} 
One starts off by considering the galactic extinction by using the Schlegel maps (Schlegel et al. 1998):

$m_{\text {extcor }}=m_{\text {sdss }}-A_{\text {Schlegel }}$,

where $m_{\text {extcor }}$ denotes the extinction correct magnitude, $m_{\text {sdss }}$ the observed apparent magnitude, and $A_{\text {Schlegel }}$ the extinction according to Schlegel maps.

The $K$-correction used in this paper,

$K\left(z_{\mathrm{obs}}, m_{f_{1}}-m_{f_{2}}\right)=\sum_{i, j} B_{i j} z_{\mathrm{obs}}^{i}\left(m_{f_{1}}-m_{f_{2}}\right)^{j}$

follows the model of Chilingarian et al. (2010), but with updated coefficients $B_{i j}$ from Saulder et al. (2013). It requires the extinction-corrected magnitudes, $m_{f_{1}}$ and $m_{f_{2}}$, of two different filters, $f_{1}$ and $f_{2}$, and the observed redshift $z_{\text {obs }}$.

In the next step, one obtains the fully corrected rest-frame magnitude $m_{\text {app }}$ by considering the $K$ correction $K\left(z_{\text {obs }}, m_{f_{1}}-\right.$ $\left.m_{f_{2}}\right)$ :

$m_{\text {cor }}=m_{\text {extcor }}-K\left(z_{\mathrm{obs}}, m_{f_{1}}-m_{f_{2}}\right)$.

The redshift $z$ is corrected for our motion relative to the cosmic microwave background (CMB).

The measured model semi-major from the SDSS data $a_{\text {sdss }}$ has to be renormalized to account for the different ellipticities of the galaxies in the following way:

$r_{\text {circ }}=a_{\text {sdss }} \sqrt{q_{b / a}}$.

We follow Bernardi et al. (2003) and get a comparable quantity for all types of elliptical galaxies, the circularized radius $r_{\text {circ }}$, with the help of the minor semi-axis to the major semi-axis ratio $q_{b / a}$.

Because of the fixed fibre size of SDSS, an additional correction on the measured central velocity dispersion $\sigma_{\text {sdss }}$ is required and we take advantage of the work of Jorgensen et al. (1995) and Wegner et al. (1999) to use

$\sigma_{0}=\sigma_{\text {sdss }} \cdot\left(\frac{a_{\text {fiber }}}{r_{\text {circ }} / 8}\right)^{0.04}$,

where $\sigma_{0}$ denotes the corrected central velocity dispersion and $a_{\text {fiber }}$ stands for the radius of the SDSS fibres, which is 1.5 arcsec for the galaxies in our sample. Here, $\sigma_{0}$ is typically about $10 \%$ higher than the measured value $\sigma_{\text {sdss }}$ (Saulder et al. 2013).

For the following calculations, one requires the luminosity distance $D_{\mathrm{L}}$, which is given by

$D_{\mathrm{L}}(z)=\frac{c \cdot z}{H_{0}}\left(1+\left(\frac{z \cdot\left(1-q_{0}\right)}{\sqrt{1+2 q_{0} \cdot z}+1+q_{0} \cdot z}\right)\right)$

with $H_{0}$ being the present day Hubble parameter and $q_{0}=$ $\frac{\Omega_{M}}{2}-\Omega_{\Lambda}$ the current declaration parameter, which depends on the cosmological parameters $\Omega_{\mathrm{M}}$ and $\Omega_{\Lambda}$.

With the luminosity distance at hand, the angular diameter distance is given by

$D_{\mathrm{A}}(z)=D_{\mathrm{L}}(z) \cdot(1+z)^{-2}$.

The physical radius $R_{0}$ of the galaxy is obtained using simple trigonometry:

$R_{0}=D_{\mathrm{A}}(z) \cdot \tan \left(r_{\text {circ }}\right)$.
The measured surface brightness $\mu_{0}$ is defined in the following way:

$\mu_{0}=m_{\text {cor }}+2.5 \cdot \log _{10}\left(2 \pi \cdot r_{\text {circ }}^{2}\right)-10 \cdot \log _{10}(1+z)+Q \cdot z$

with the term $-10 \cdot \log _{10}(1+z)$ correcting for cosmological dimming of surface brightnesses. Since we only intend to use the surface brightness, we include a parameter that corrects for the secular evolution evolution of early-type galaxies, when applying or calculating the fundamental plane (see Appendix A and Saulder et al. 2013). The evolution parameter $Q=1.07$ mag per $z$ was derived in Saulder et al. (2013) for early-type galaxies.

Another quantity required for our investigations is the absolute magnitude $M_{\mathrm{abs}}$, which is calculated using the distance module:

$m_{\text {cor }}-M_{\text {abs }}=5 \cdot \log _{10}\left(D_{\mathrm{L}} / \mathrm{pc}\right)-5$.

The dynamical mass is given by

$M_{\mathrm{dyn}}=\frac{\beta(n) \sigma_{0}^{2} \cdot R_{0}}{G}$

with $G$ being the gravitational constant. The function $\beta\left(n_{\mathrm{S}}\right)$ is defined by:

$\beta(n)=8.87-0.831 \cdot n_{\mathrm{S}}+0.0241 \cdot n_{\mathrm{S}}^{2}$

according to Cappellari et al. (2006), based on results from Bertin et al. (2002). It depends on the Sersic-index $n_{\mathrm{S}}$, if a Sersic profile was used to obtain the effective radius. For de Vaucouleurs profiles, which are Sersic profiles with Sersicindices $n_{\mathrm{S}}=4$, one would expect a $\beta$ of 5.953; however, it has been found by observations (Cappellari et al. 2006) that a $\beta$ of five works better. Cappellari et al. (2006) argue that this deviation is due to differences between the idealised simulation Eq. (12) is based on and real observational data. Belli et al. (2014) find that the equations works well for spherical systems, but has problems if discs are present. We therefore decided to use the $\beta\left(n_{\mathrm{S}}\right)$ from Eq. (12), when using a Sersic profile, but we adopt a $\beta$ of 5 , when using a de Vaucouleurs profile in our analysis.

With all the equations and definitions given in this section, we now proceed to the selection and analysis of galaxies similar to b19.

\section{Candidate selection}

The main idea behind this paper is to find galaxies with properties comparable to b19 (Läsker et al. 2013) and NGC 1277 (van den Bosch et al. 2012) and to investigate whether they are unique objects or not. B19 is characterized by a relatively small scale radius, but a relatively high central velocity dispersion that implies a high dynamical mass for its given radius.

In the following we define a set of criteria that provides us with galaxies in the same region of the $\log _{10}\left(R_{0}\right)-\log _{10}\left(\sigma_{0}\right)$ diagram as b19. The selection criteria have to be restrictive enough that only the most massive and most compact galaxies are included, but still generous enough to include b19. To avoid too much arbitrariness, we used the samples averages and standard deviations as a basis for our definitions. We adopted the following selection criteria:

- $\log _{10}\left(R_{0}\right)<\overline{\log _{10}\left(R_{0}\right)}-\sigma_{\log _{10}\left(R_{0}\right)}$

$-\log _{10}\left(\sigma_{0}\right)>\overline{\log _{10}\left(\sigma_{0}\right)}+2 \sigma_{\log _{10}\left(\sigma_{0}\right)}$

- $\log _{10}\left(\sigma_{0}\right)-k_{R \sigma} \cdot \log _{10}\left(R_{0}\right)<d_{R \sigma}+3 s_{\epsilon, R \sigma}$. 


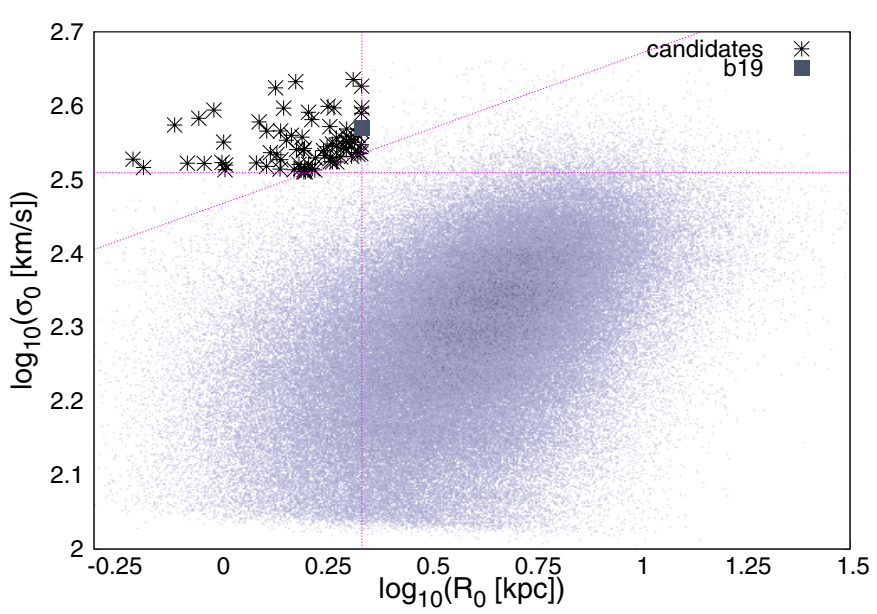

Fig. 1. Selection criteria for our compact and massive galaxy candidates indicated by the dashed magenta lines. The black stars represent the 75 new candidates for galaxies with similar properties in de Vaucouleurs fit parameters as b19, while b19 itself is represented by a grey filled square in the plot.

The first criterion means that the logarithm of the physical radius $R_{0}$ has to be smaller than the sample's average $\overline{\log _{10}\left(R_{0}\right)}$ by at least one standard deviation $\sigma_{\log _{10}\left(R_{0}\right)}$, which provides us with an upper limit for $R_{0}$ of $2.18 \mathrm{kpc}$ for the de Vaucouleurs fit parameters. The lower limit for the central velocity dispersion $\sigma_{0}$ is requiring by demanding it to be at least two standard deviation $\sigma_{\log _{10}\left(\sigma_{0}\right)}$ higher than the mean of the logarithm of the central velocity dispersion $\overline{\log _{10}\left(\sigma_{0}\right)}$. This yields a lower limit of $\sigma_{0}=323.2 \mathrm{~km} \mathrm{~s}^{-1}$. The last criterion ensures that all candidates are more than three root mean square $s_{\epsilon, R \sigma}$ off from the $\log _{10}\left(R_{0}\right)-\log _{10}\left(\sigma_{0}\right)$ relation: $\log _{10}\left(\sigma_{0}\right)=k_{R \sigma} \cdot \log _{10}\left(R_{0}\right)+d_{R \sigma}$, for which the coefficients $k_{R \sigma}$ and $d_{R \sigma}$ were obtained by a linear fit to the data points of the basic sample. The selection criteria are illustrated in Fig. 1.

By applying the above selection criteria to the basic sample, one finds 76 galaxies. All candidates are listed with their basic parameters in Table 2 and their derived parameters in Table 3. B19 itself has the internal ID 2. The others are new compact massive galaxies similar to b19, whose global properties will be investigated over the course of this paper. A set of SDSS thumbnail images for all our candidates is provided in Fig. 2.

In Appendix B, we provide an alternative sample of candidates using the Sersic fit parameters from Simard et al. (2011) instead of the de Vaucouleurs fit directly from SDSS.

\section{Results}

In this section, we discuss the distribution of our candidates along known scaling relations for early-type galaxies. We compare our sample to the work of Taylor et al. (2010), who listed 63 compact massive red-sequence galaxies in a similar redshift range. When cross-matching their sample with our data, we find 60 of their galaxies that are in our basic sample. Another sample of possible low-redshift, compact, massive red-sequence galaxies is the sample of Trujillo et al. (2009), which contains 29 such galaxies, of which we detect 23 in our basic sample. All samples are based on SDSS. In the following, we compare our compact galaxy sample of 76 galaxies to the 60 galaxies that are in both our basic sample and the Taylor et al. (2010) sample, as well as to the 23 galaxies, which are in both our basic sample and the
Trujillo et al. (2009) sample. For simplicity, we call the 60 galaxies of Taylor et al. (2010), which are in our basic sample, the Ta10 sample from here on, and they are listed with their basic and derived parameters from SDSS in Tables C.2 and C.3. The 23 galaxies of the Trujillo et al. (2009), which are in our basic sample, are called the Tr09 sample from here, and they are listed with their basic and derived parameters from SDSS in Tables C.4 and C.5.

A comparison of the Ta10 sample with our compact galaxy sample reveals that they only have five galaxies in common (see Table C.1). It is surprising to only find so few galaxies in common with a sample that should be similar to our own. The difference between our candidate sample and the Tr09 is even more striking, since they do not share a single galaxy. Aside from local samples, we compare our candidates also to various samples of intermediate-and high redshift data (see Figs. 5 and following). We used the recent intermediate redshift sample of Zahid et al. (2015), the classic high redshift sample of Damjanov et al. (2009), the new high redshift sample of Belli et al. (2014), and the catalogue of van de Sande et al. (2013), which contains a composition of various high redshift samples, such as Bezanson et al. (2013), van Dokkum et al. (2009), Onodera et al. (2012), Cappellari et al. (2009), Newman et al. (2010), van der Wel et al. (2008), Blakeslee et al. (2006), Toft et al. (2012), and their own work. We cannot perform a comparison with these datasets in every figure, because sometimes some samples do not contain the required parameters.

\subsection{The fundamental plane}

As illustrated in Fig. 3, the fundamental plane is a tight relation for early-type galaxies and a good starting point for our investigation. According to Läsker et al. (2013), b19 is a clear outlier of the fundamental plane of Bernardi et al. (2003). In contrast to this, we found that b19 is only slightly more than $1 \sigma$ off the fundamental plane using the new coefficients listed in Appendix A, which are based on the work of Saulder et al. (2013). Furthermore, all candidate galaxies can be found clearly within $3 \sigma$ of the fundamental plane (see Fig. 3). Almost all of them are located on the same side above the fundamental plane and are grouped in a similar region. The Ta10 sample is much more distributed over the fundamental plane than our sample. Some galaxies in the Ta10 sample are even beyond the $3 \sigma$ boundary on the opposite side to the clustering of our candidates. The Tr09 sample forms a relatively tight group around and beyond the $3 \sigma$ boundary at the opposite side of our candidate sample on the fundamental plane. The Ta10 sample appears to be distributed between the Tr09 sample and our sample, which are opposite extremes of the Ta10 sample distribution.

\subsection{The colour-magnitude diagram}

In Fig. 4, we plot the $z$ band absolute magnitudes vs. the $g-r$ colours. Galaxies in the colour-magnitude plane can generally be divided into two main groups: the red sequence and the blue cloud (Chilingarian \& Zolotukhin 2012), which are only connected by a relatively sparsely populated "green valley". While the blue cloud is mainly composed of late-type galaxies, the red sequence mainly consists of early-type galaxies, such as the galaxies discussed in this paper. The selection criteria of our basic sample reduces the galaxies used in this paper to the red sequence. In Fig. 4 we indicate the red sequence fit from 


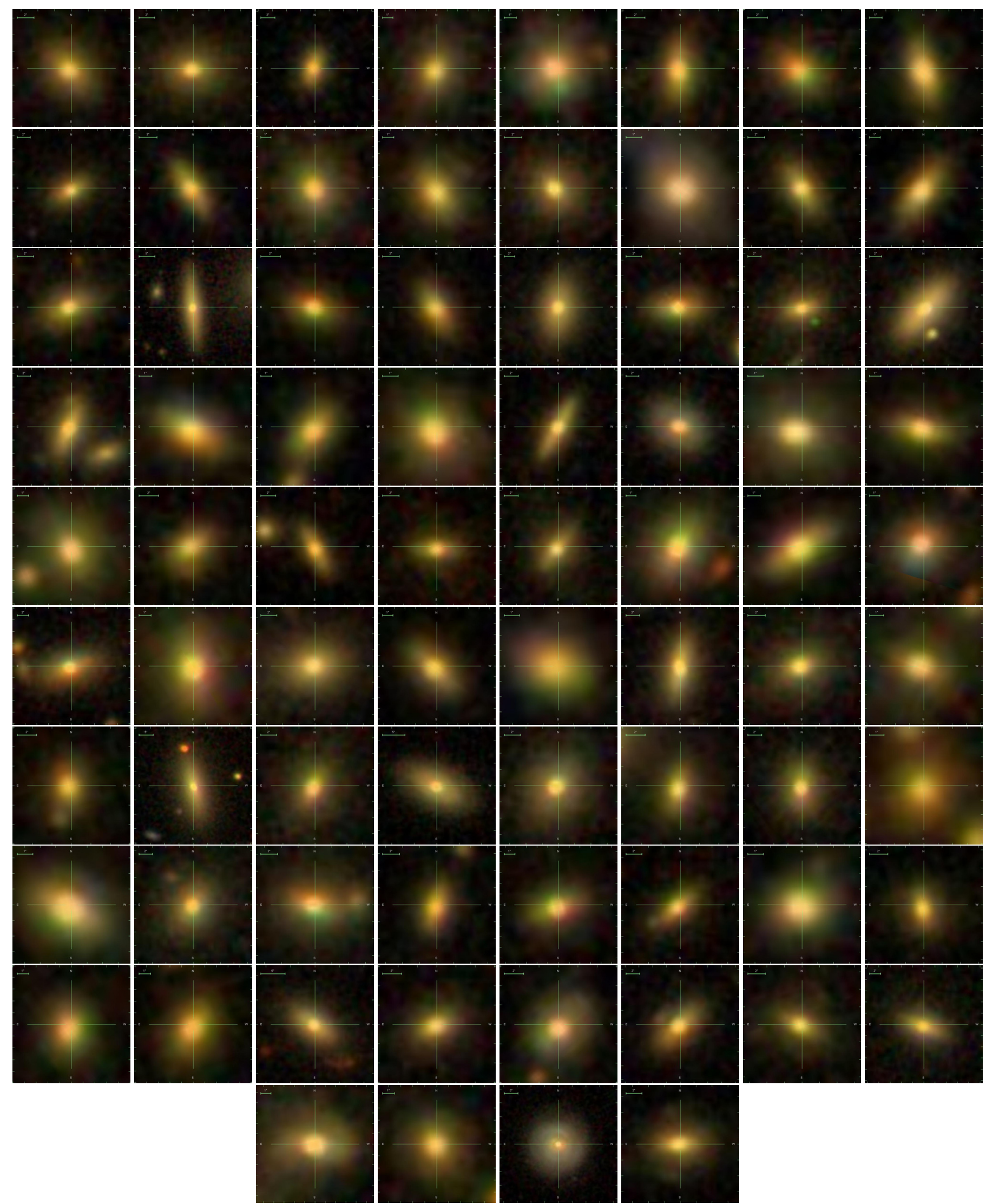

Fig. 2. SDSS DR10 colour thumbnails for our 76 compact massive galaxy candidates. They are arranged by their internal ID with galaxy 1 in upper left corner and then in ascending order from left to right and top to bottom. B19 is the second galaxy in the top row. The thumbnails show an square area with a side length corresponding to $12 a_{\text {sdss }}$ of the displayed galaxy. There is also a small scale in the top left corner of each image. 


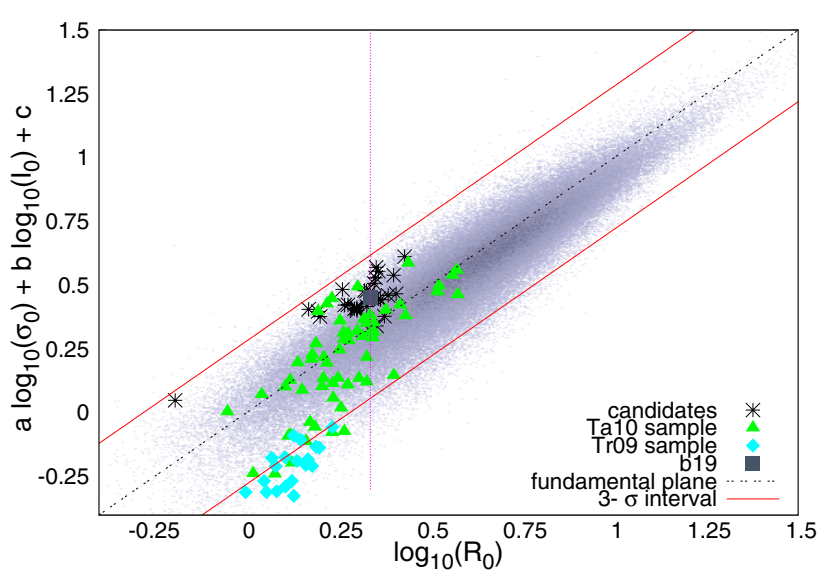

Fig. 3. Location of the candidate galaxies on the fundamental plane. The candidates are indicated by black stars. The galaxies belonging to the Ta10 sample are represented using filled green triangles, and the Tr09 sample is denoted by filled cyan diamonds. B19, the starting point of our investigation, is indicated by a filled grey square. The magenta dotted lines show the limiting physical radius used in the sample sample selection. The black dashed lines are the fundamental plane fits from Appendix A with their corresponding $3 \sigma$ confidence intervals shown as red solid lines. The fit appears to be slightly offset due to the volume weights used to correct the Malmquist bias in the fitting process.

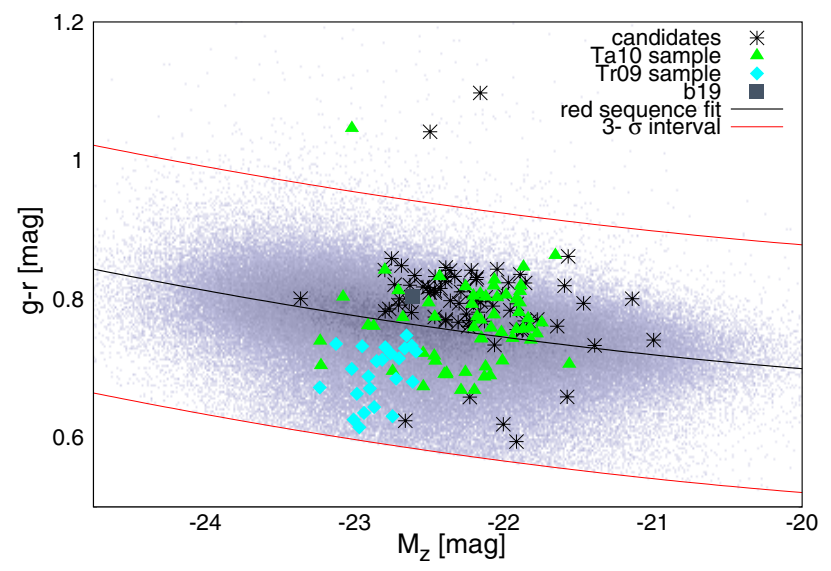

Fig. 4. Distribution of the candidate galaxies in a colour-magnitude diagram. The galaxies belonging to the Ta10 sample are represented using filled green triangles, and the Tr09 sample is denoted by filled cyan diamonds. B19 is indicated by a filled grey square. The black dashed line represents the fit on the red sequence performed in Saulder et al. (2013) with the corresponding $3 \sigma$ confidence intervals shown as solid red lines.

Saulder et al. (2013) in the $g-r$ colour vs. the absolute $z$ band magnitude plane.

At a given absolute magnitude $M_{z}$, the galaxies of our candidate sample are systematically redder than the average red sequence galaxy by about $0.05 \mathrm{mag}$ in $g-r$. At the same time, except for two outliers, all galaxies are well within $3 \sigma$ limits of the overall distribution, and there are only a few galaxies, which are blue than the average red sequence galaxy. Most of the galaxies of the Ta10 sample are also redder than the average and are associated to the grouping of galaxies from the candidates (see Fig. 4). The systematic offset of our sample towards redder colours is consistent with a higher stellar metallicity than that of the average early-type galaxy at the same luminosity. All galaxies of the Tr09 sample are bluer than the average red sequence galaxy, which contrasts with our candidate sample.

\subsection{The mass-size relations}

In Figs. 5 and 6, we plot the stellar masses and the dynamical masses, respectively, against the physical radii of the galaxies. Relations between the size and the mass of dynamically hot stellar systems are frequently used to distinguish them into different classes, as done, for example, in Misgeld \& Hilker (2011) for dwarf galaxies vs. star clusters. Also bulges, large elliptical galaxies and similar objects can be found in very distinct areas of a mass-size diagram. The galaxies in which we are interested in this paper are early-type galaxies with small radii and relatively high masses. These galaxies are located on the edge of the socalled zone of exclusion (Burstein et al. 1997; Misgeld \& Hilker 2011; Norris et al. 2014). This zone is empirically defined by a limit of stellar mass beyond which (most) hot stellar systems cannot grow at fixed sizes.

In Fig. 5, one finds, in contrast to the previous figures, that there seems to be rough overall agreement on the distribution of our galaxies and the galaxies from Taylor et al. (2010) as well as our galaxies and the galaxies from Trujillo et al. (2009). We found that the galaxies from the Ta10 sample tend to contain less stellar mass for their sizes than our candidates. In contrast, the galaxies of the Tr09 sample tend to be more compact for their stellar masses than most of our galaxies. Almost all galaxies are at the edge of the distribution, as expected. When plotting the dynamical mass instead of the stellar mass against the scale radius (see Fig. 6), the Ta10 and the Tr09 samples are detached from our candidates again. Since Fig. 6 is basically a rescaled and tilted version of the selection criteria (see Fig. 1), because of the definition of the dynamical mass (see Eq. (11)), it highlights the differences in the sample selection between this work and Taylor et al. (2010) as well as Trujillo et al. (2009), who used stellar masses, when compared to Fig. 5. Over the course of this paper, we found that our selection criteria yield a more cohesive sample than the Ta10 sample or the Tr09 sample. The sample of Zahid et al. (2015) apparently contains many galaxies with larger radii than the low redshift samples. Most galaxies in the various high redshift samples can be found in areas close to our candidates and the other low redshift sample. They are located close to the edge of the zone of exclusion. A more detailed discussion of the differences between our sample and the samples of various other authors can be found in Sects. 6.4 and 6.5.

\subsection{The mass-to-light ratio}

Since the starting point of our investigation, b19, is said (Läsker et al. 2013) to have a bottom-heavy initial mass function, the mass-to-light ratio $\uparrow$ will contain valuable information for us. We investigated both the dynamical mass-to-light ratio $\Upsilon_{\text {dyn }}$ and the stellar mass-to-light ratio $\Upsilon_{*}$. The dynamical mass-to-light ratio is derived directly from measured SDSS parameters using Eq. (11), while the stellar mass-to-light ratio requires some additional modelling to derive the stellar masses, which was done by Mendel et al. (2014). They used a stellar population synthesis to derive the stellar masses from spectral energy distributions based on the SDSS broadband photometry.

Figures 7 and 8 illustrate the distribution of the $r$ band dynamical and stellar mass-to-light ratios, respectively, for our candidate sample, the Ta10 sample, the Tr09 sample, and the basic sample. Comparing the mass-to-light ratios of our candidates to the basic sample, we found them clearly elevated. The average dynamical mass-to-light of the basic sample is $3.75 \pm 0.46 M_{\odot} / L_{\odot, r}$, and the average stellar mass-tolight of the basic sample is $2.07 \pm 0.20 M_{\odot} / L_{\odot, r}$. The average 


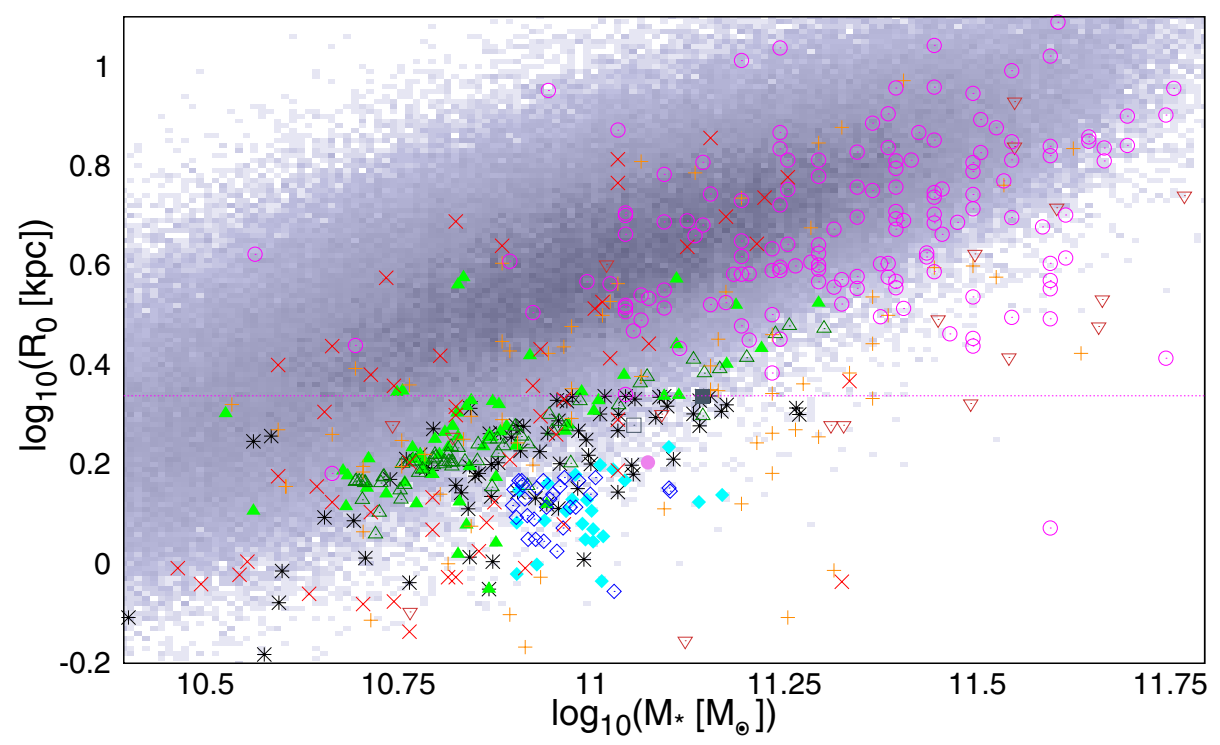

candidates (this paper)

Ta10 sample

Tr09 sample

Taylor+ $2010 \triangle$

Trujillo+ $2009 \diamond$

van de Sande +2013

Belli+ 2014

Damjanov+ 2009

Zahid+ 2015

van den Bosch+ 2012

b19 (this paper)

b19 (Lasker+ 2013)

Fig. 5. Stellar mass-size relation for our basic sample and several other samples of compact massive early-type galaxies from the literature in comparison to our own data. The blueish cloud indicates the early-type galaxies of our basic sample. The black stars represent the candidates of our sample. The galaxies of the Ta10 sample are shown using filled green triangles and the galaxies of the Tr09 sample are denoted by filled cyan diamonds. The galaxies from Taylor et al. (2010), using the values of their paper, are indicated by open dark green triangles. The open blue diamonds represent the galaxies of Trujillo et al. (2009). Orange crosses mark the catalogue of various high redshift samples by van de Sande et al. (2013). The high redshift sample of Belli et al. (2014) is indicated by red Xs. Open brown nabla symbols mark the high redshift galaxies of Damjanov et al. (2009). Open magenta circles indicate the intermediate redshift sample of Zahid et al. (2015). NGC 1277 of van den Bosch et al. (2012), which is the only galaxy of their sample for which we have a stellar mass is represented by an filled violet circle. b19 using our calibration of SDSS data is shown by a filled grey square and b19 using the calibration of Läsker et al. (2013) is indicated by an open grey square. Because we use the values available in the literature to mark the positions of the galaxies in this plot, one has to consider potential systematics, especially in the effective radius $R_{0}$, which was measured in different filters by different authors. The dashed magenta line marks the limiting scaling radius for our sample selection.

dynamical mass-to-light ratio of our candidate sample is $7.60 \pm$ $2.45 M_{\odot} / L_{\odot, r}$, which is about twice the number of the average of the basic sample. Also the average stellar mass-to-light ratio of our candidates is with, $2.66 \pm 0.38 M_{\odot} / L_{\odot, r}$, notably higher than the one of the basic sample. The average mass-to-light ratios of the Ta10 sample are, however, relatively close to the averages of the basic sample with a $\Upsilon_{\text {dyn }}$ of $3.81 \pm 1.98 M_{\odot} / L_{\odot, r}$ and a $\Upsilon_{*}$ of $2.27 \pm 0.51 M_{\odot} / L_{\odot, r}$. The average mass-to-light ratios of the Tr09 sample are extremely low: $\Upsilon_{\text {dyn }}=1.15 \pm 0.31 M_{\odot} / L_{\odot, r}$ and $\Upsilon_{*}=1.60 \pm 0.24 M_{\odot} / L_{\odot, r}$.

Conroy et al. (2013) show that there is an increasing difference between the dynamical and the stellar mass-to-light ratio for compact galaxies with higher central velocity dispersion using the same data (fits from Simard et al. 2011 and stellar masses from Mendel et al. 2014). They argue that this indicates a systematic variation in the initial mass function. In Fig. 9, we plot the ratio of dynamical over stellar mass against velocity dispersion. The increase in this ratio with increasing velocity dispersion is clearly visible. The area in Fig. 9 below a logarithm of the dynamical-mass-to-stellar-mass ratio of zero is only sparsely populated, and most galaxies in that region are consistent with a $\log$ ratio of 0 thanks to measurement uncertainties $(0.15 \mathrm{dex}$ for the stellar masses according to Mendel et al. 2014). The Ta10 sample is scattered widely over the general distribution with some galaxies even in the forbidden area, while our candidates form the high end in Fig. 9 owing to our selection criteria. We found that the galaxies of our sample have a $M_{\text {dyn }}$ to $M_{*}$ ratio as one might expect for galaxies with such high $\sigma_{0}$, following the general trend of the galaxy distribution in Fig. 9. Galaxies of the basic sample with a central velocity dispersion between 323.2 and $400.0 \mathrm{~km} \mathrm{~s}^{-1}$ have $\log _{10}\left(M_{\text {dyn }} / M_{*}\right)=0.432$, which is almost the same value as our candidates with $\log _{10}\left(M_{\text {dyn }} / M_{*}\right)=0.441$. The vast majority of the Tr09 sample have $M_{\text {dyn }}$ to $M_{*}$ ratios below one and are thus located in a zone of exclusion, indicating possible problems in the measurement of the stellar masses of these galaxies. Although the galaxies of the various intermediate and high redshift samples are scattered widely the distribution of our basic sample, there is a tendency toward higher central velocity dispersion, but few of them reach values as high as our candidates.

In Fig. 10, we plot the distribution of our sample in the dynamical mass vs. stellar mass plane. The difference between our own sample and the Ta10 sample becomes very clear in Fig. 10. While the Ta10 sample has several objects with lower dynamical to stellar mass-to-light ratios, our galaxies are in general more massive in both dynamical and stellar mass, and they show a tendency for elevated dynamical mass compared to their stellar mass, as already shown in Fig. 9. The Tr09 sample is again concentrated in what is normally a zone of exclusion, with formally higher stellar than dynamical masses.

\subsection{The Sersic indices}

Thanks to the SDSS refits from Simard et al. (2011), we have Sersic profiles for almost all SDSS galaxies. In Fig. 11, we compare the Sersic indices of different samples. It should be pointed out that the algorithm used by Simard et al. (2011) only allows a maximum Sersic index $n=8$, so we have some clustering around this value for all samples. In Fig. 11 there is a clear difference between our basic sample, which only consists of red sequence galaxies because of the colour cut and GalaxyZoo 


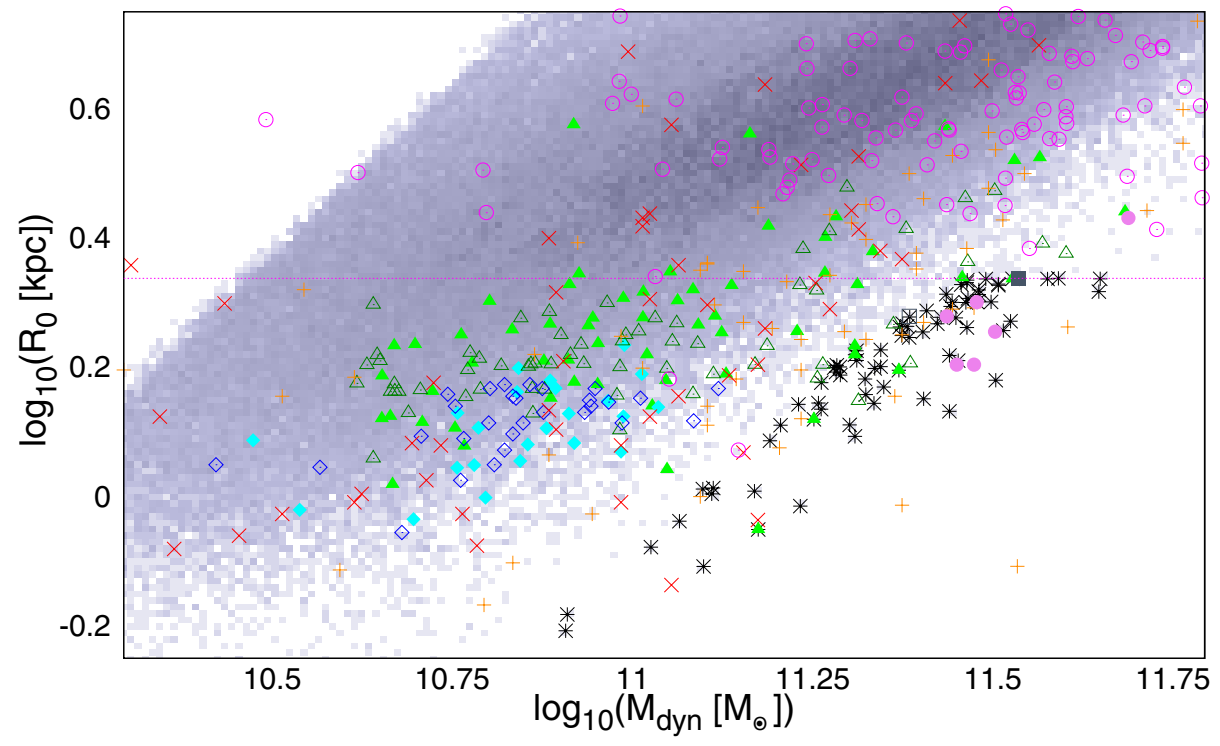

candidates (this paper)

Ta10 sample

Tr09 sample

Taylor+2010 $\triangle$

Trujillo+ $2009 \diamond$

van de Sande +2013

Belli+ 2014

Zahid+ 2015

van den Bosch +2012

b19 (this paper)

b19 (Lasker+ 2013)

Fig. 6. Dynamical mass-size relation for our basic sample and several other samples of compact massive early-type galaxies from the literature in comparison to our own data. The blueish cloud indicates the early-type galaxies of our basic sample. The black stars represent the candidates of our sample. The galaxies of the Ta10 sample are shown using filled green triangles and the galaxies of the Tr09 sample are marked by filled cyan diamonds. The galaxies from Taylor et al. (2010) using the values of their paper are indicated by open dark green triangles. The open blue diamonds represent the galaxies of Trujillo et al. (2009). Orange crosses mark the catalogue of various high redshift samples by van de Sande et al. (2013). The high redshift sample of Belli et al. (2014) is indicated by red Xs. We do not have dynamical masses for the high-redshift sample of Damjanov et al. (2009). However we can calculate dynamical masses for the intermediate-redshift sample of Zahid et al. (2015), which is indicated by open magenta circles. The six galaxies of van den Bosch et al. (2012) are represented by filled violet circles. b19 using our calibration of SDSS data is shown by a filled grey square and b19 using the calibration of Läsker et al. (2013) is indicated by an open grey square. Because we use the values available in the literature to mark the positions of the galaxies in this plot, one has to consider potential systematics, especially in the effective radius $R_{0}$, which was measured in different filters by different authors. The dashed magenta line denotes the limiting scaling radius for our sample selection.

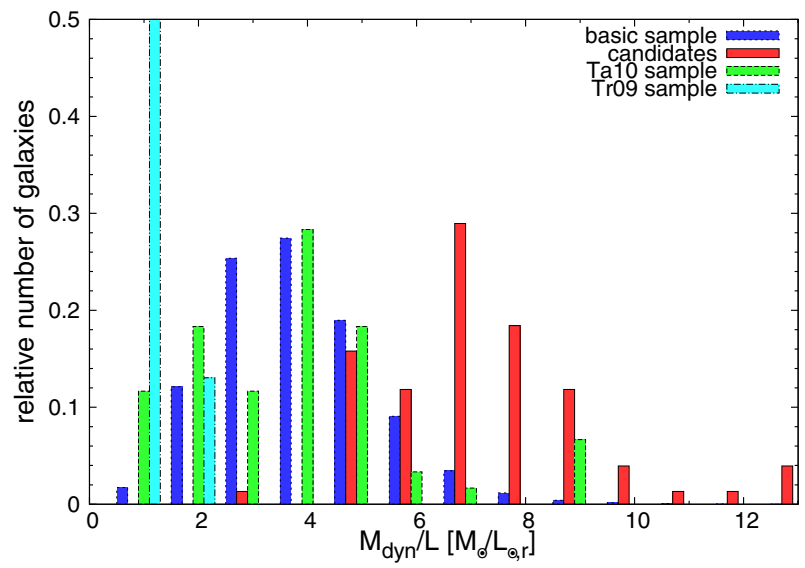

Fig. 7. Distribution of the dynamical mass-to-light ratios $\Upsilon_{\text {dyn }}$. The blue histogram corresponds to our basic sample, which consists of early-type galaxies alone.The green histogram represents the Ta10 sample, while the cyan histogram corresponds to Tr09 sample. The red histogram indicates our 76 candidates.

classification (Lintott et al. 2011) used in its selection, and the sample of all SDSS DR7 galaxies, that qualified for the refits done by Simard et al. (2011), which thereby consists of a mixed population. The early-type galaxies have clearly higher Sersic indices than the full SDSS DR7 sample. The Ta10 sample and our 76 candidates do not show any significant peculiarities compared to the distribution of the Sersic indices of the basic sample, except for a less smooth distribution due to small number statistics and a weak trend toward higher Sersic indices. The

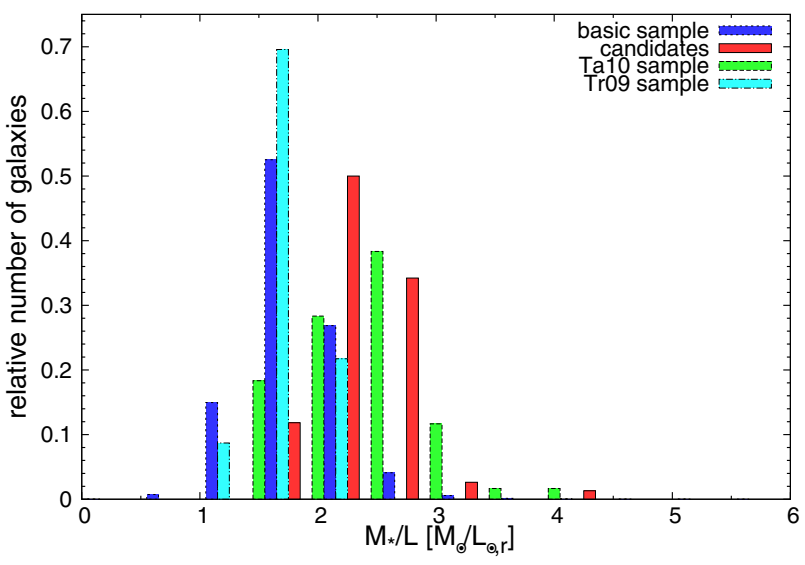

Fig. 8. Distribution of the stellar mass-to-light ratios $\Upsilon_{*}$. The blue histogram corresponds to our basic sample, which consists of early-type galaxies alone. The green histogram represents the Ta10 sample, while the cyan histogram corresponds to Tr09 sample. The red histogram indicates our 76 candidates.

Tr09 sample shows an outstanding peak in its distribution around a Sersic index of 4.5, but is otherwise in agreement with our basic sample. The sample of Belli et al. (2014) shows a preference for lower Sersic indices in contrast to all other samples.

\subsection{The semi-axis ratios}

We investigate the distribution of the semi-minor to semi-major axis ratios $q_{b / a}$ of our sample and the samples that we used for 


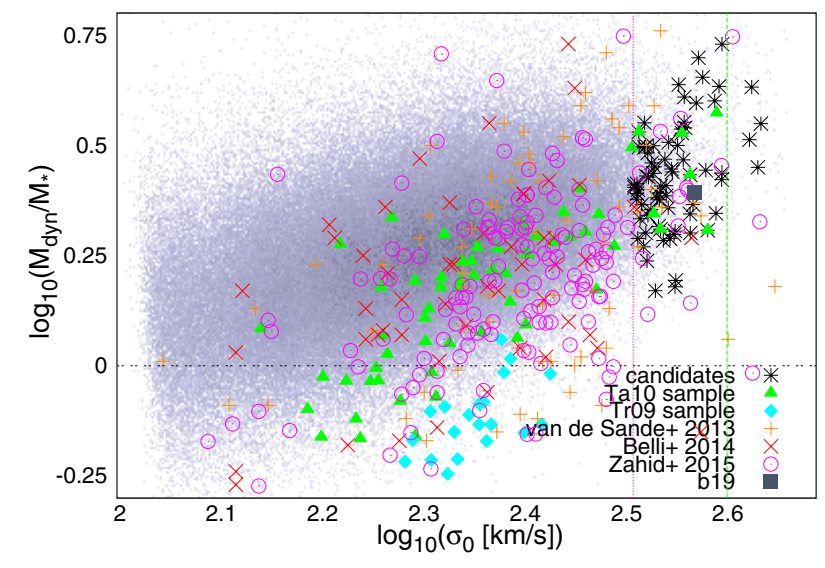

Fig. 9. Dependence of the dynamical mass $M_{\text {dyn }}$ to stellar mass $M_{*}$ ratio on central velocity dispersion $\sigma_{0}$. The candidates are indicated by black stars. The galaxies belonging to the Ta10 sample are represented using filled green triangles, and the $\operatorname{Tr} 09$ sample is indicated by filled cyan diamonds. Orange crosses indicate the catalogue of various high redshift samples by van de Sande et al. (2013). The high redshift sample of Belli et al. (2014) is indicated by red Xs. The intermediate redshift sample of Zahid et al. (2015) is indicated by open magenta circles. B19 is indicated by a filled grey square. The magenta dashed line marks the limiting scaling central velocity dispersion for our sample selection. The green dashed line indicates a central velocity dispersion of $400 \mathrm{~km} \mathrm{~s}^{-1}$. The area below the black dashed line is considered to be unphysical, because $M_{*}$ would exceed $M_{\mathrm{dyn}}$.

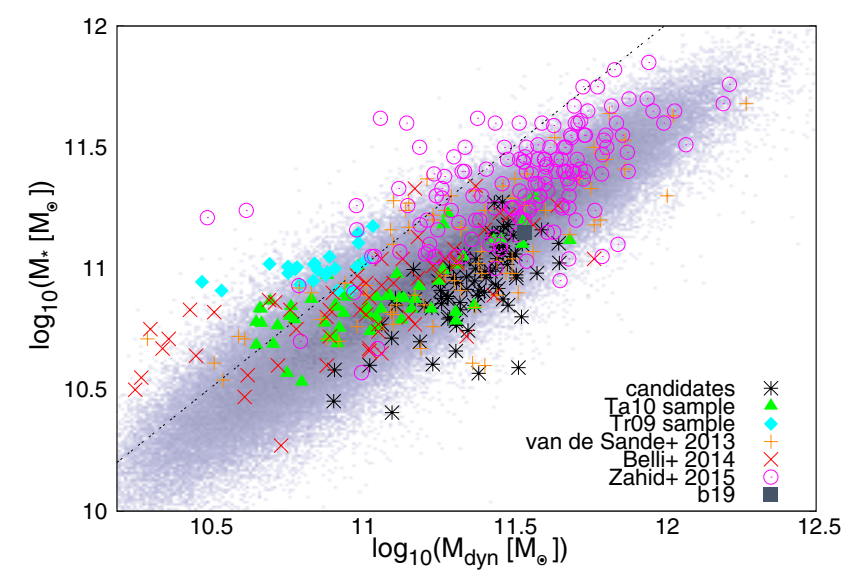

Fig. 10. Distribution of the sample's galaxies in the dynamical mass $M_{\text {dyn }}$ vs. stellar mass $M_{*}$ plane. The candidates are indicated by black stars. The galaxies belonging to the Ta10 sample are represented using filled green triangles, and the Tr09 sample is indicated by filled cyan diamonds. Orange crosses indicate the catalogue of various high redshift samples by van de Sande et al. (2013). The high redshift sample of Belli et al. (2014) is indicated by red Xs. The intermediate redshift sample of Zahid et al. (2015) is indicated by open magenta circles. $\mathrm{B} 19$ is indicated by a filled grey square. The magenta dashed line marks the limiting scaling central velocity dispersion for our sample selection. The black dashed line marks the limit of the $M_{\mathrm{dyn}}$ to $M_{*}$ ratio, which is still considered to be physical, because above it $M_{*}$ would exceed $M_{\mathrm{dyn}}$.

comparison. A ratio of 1 indicates a perfectly round galaxy, and the value decreases to zero for increasing ellipticity of the galaxy. Our candidates are clearly more elongated than the galaxies of our basic sample. The Ta10 sample appears to be rounder than the basic sample, but the Tr09 contains several galaxies with high ellipticity (higher than our sample), but also a larger number of very round galaxies than our sample. The

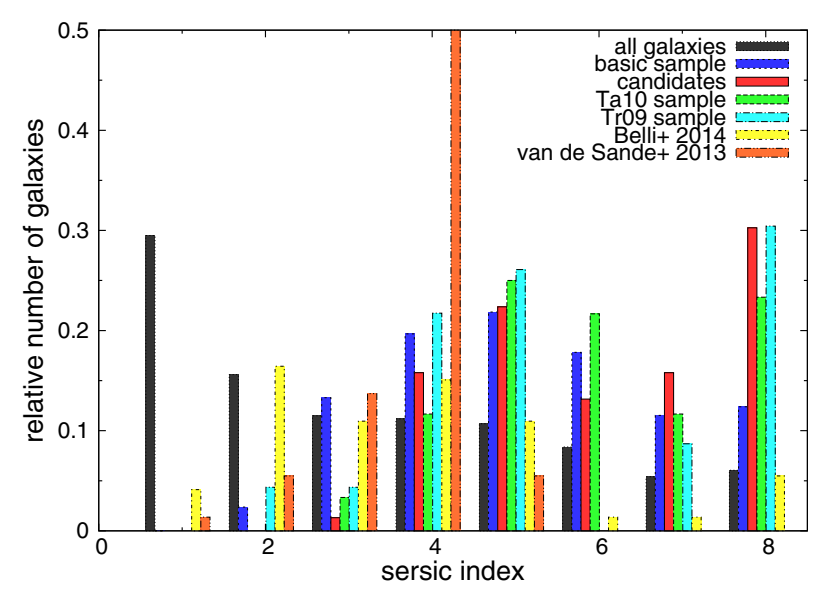

Fig. 11. Distribution of the Sersic indices of different samples of galaxies. The black histogram corresponds to all galaxies in SDSS DR7 for which Simard et al. (2011) did their refits. The blue histogram corresponds to our basic sample, which consists of early-type galaxies alone. The green histogram represents the Ta10 sample, and the cyan histogram the Tr09 sample. The yellow histogram corresponds to sample of Belli et al. (2014) and the orange histogram indicated the distribution of the sample of van de Sande et al. (2013). The red histogram shows our 76 candidates.

sample of Belli et al. (2014) seems to contain on average rounder galaxies than our candidate sample.

\section{Discussion}

We started our investigation with b19, which is known to be a very compact massive elliptical galaxy in SDSS in the local universe (Läsker et al. 2013). In this paper, we identify a sample of 76 galaxies (including b19) that have similar global properties, with dispersions of $\sigma_{0}>323.2 \mathrm{~km} \mathrm{~s}^{-1}$ and sizes smaller than $R_{0}<2.18 \mathrm{kpc}$. These selection criteria, which are described in detail in Sect. 4, place these objects at the edge of the $\log _{10}\left(R_{0}\right)-\log _{10}\left(\sigma_{0}\right)$.

\subsection{Global properties}

Our candidate sample forms a relatively homogeneous group in most of the scaling relations and parameter spaces we investigated. The observed parameters (see Table 2), such as the observed apparent magnitude $m_{\text {sdss }}$, the angular semi-major axis $a_{\text {sdss }}$, and the central velocity dispersion $\sigma_{\text {sdss }}$, are in a range where SDSS measurements are reliable (statistical error of less than $4 \%$ ).

We found that all our candidate galaxies are located within the $3 \sigma$ limits of the fundamental plane using the new coefficients listed in Appendix A. They occupy the same corner of the overall distribution of the galaxies on the fundamental plane (see Fig. 3), indicating that our candidates are more compact than the average galaxy with similar surface brightness and central velocity dispersion.

Our candidates are also redder than the average early-type galaxies, suggesting metallicity enhancements compared to less compact galaxies at the same luminosity. The vast majority of them can be found above our fit on the red sequence in the colour-magnitude diagram (see Fig. 4), but still within the $3 \sigma$ limits (except for two very red outliers).

When analysing the mass-size relations, we found that the galaxies of the candidate sample are located within or close to 


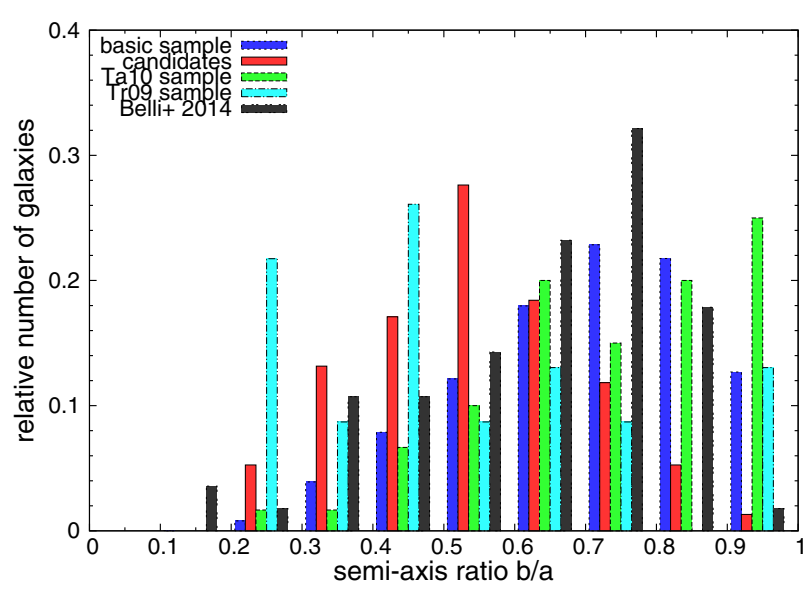

Fig. 12. Distribution of the semi-minor to semi-major axis ratios $q_{b / a}$ of different samples of galaxies. The blue histogram corresponds to our basic sample. The green histogram represents the Ta10 sample, and the cyan histogram the Tr09 sample. The black histogram corresponds to sample of Belli et al. (2014). The red histogram shows our 76 candidates.

the zone of exclusion (Burstein et al. 1997; Misgeld \& Hilker 2011; Norris et al. 2014). This is a direct consequence of the sample's definition, because we were looking specifically for galaxies in this area. We wanted the most massive galaxies for their given small sizes, and Figs. 5 and 6 illustrate nicely that we got them. One should keep in mind that the sizes may carry an additional systematic uncertainty, because the intrinsic angular sizes of our galaxies are close to the size of the SDSS PSF.

We found that both the stellar and the dynamical mass-tolight ratios of our candidates are elevated compared to the rest of the sample. Figure 9 illustrates that there is an increasing difference between the stellar masses and the dynamical masses with increasing central velocity dispersion $\sigma_{0}$ as reported in Conroy et al. (2013). Since we specifically selected for high central velocity dispersion galaxies, the candidates belong to the high $\sigma_{0}$ tip of this correlation. Our galaxies have not only high stellar and dynamical masses for their small sizes, but also high dynamical masses for their given stellar mass as illustrated in Fig. 10.

As shown in Fig. 11, our sample does not have any significant difference in their Sersic indices compared to regular early-type galaxy. Figure 12 shows that our candidate galaxies have a higher ellipticity than the galaxies of the basic sample. In the context of the SAURON results (Krajnović et al. 2008; Cappellari et al. 2007; Emsellem et al. 2007), we interpret these results such that most of our our candidates are lenticular galaxies that host a significant disc component. This result is consistent with the observation that red nuggets are disc-dominated (van der Wel et al. 2011; Chevance et al. 2012).

\subsection{Comparison to the Ta10 sample}

The most important difference between our candidate sample and the sample of Taylor et al. (2010) is the way in which it was selected. Taylor et al. (2010) selected their sample using a colour cut demanding that their galaxies be redder than than 2.5 in ${ }^{0.1}(u-r)^{5}$, and they also restricted their sample to have a minimum stellar mass of $10^{10.7} M_{\odot}$. Furthermore, their galaxies have to be located between the redshifts of 0.066 and 0.12 , while our candidate sample allows redshifts up to 0.4, although

\footnotetext{
5 This denotes the SDSS $u-r$ band colour at a redshift of 0.1 .
}

we did only detect one galaxy beyond 0.2 . When cross-matching their 63 galaxies with our own basic sample, we found 60 galaxies. These 60 galaxies, the so-called Ta10 sample, are listed with their parameters in Tables C.2 and C.3. There are only five galaxies that are shared between the Ta10 sample and our candidate sample (see Table C.1), the galaxies with the internal IDs 6, 13, 39,52 , and 53 . We attribute the difference between our candidates and the Ta10 sample to the different selection criteria and want to point out that most of our galaxies tend to contain more stellar mass and definitely more dynamical mass for their sizes than the galaxies of the Ta sample.

In general, we found that the galaxies of the Ta10 sample occupy different regions in the various considered scaling relations and diagrams than our candidate galaxies. Furthermore, they are a less homogeneous sample than the galaxies presented in this paper. In a stellar mass-size diagram (see Fig. 5), they occupy a very similar corner to our candidates, although they are less massive for their size than a large portion of our galaxies. Their distribution in this specific diagram is restricted to a small region, which is a consequence of the selection criteria for this sample. Figure 5 also contains the positions in the stellar mass-size plane of all galaxies of Taylor et al. (2010) using the parameters of their paper. They occupy a similar area in that plot. Comparing Figs. 5 and 6 highlights the difference between the Ta10 sample and our candidates owing to their selection criteria. We found that the galaxies of the Ta10 sample largely behave like average early-type galaxies, while our candidates always occupy offaverage regions in the parameter space, because they are in the extreme tail of the general distribution of early-type galaxies. We note, though, that there is a small overlap of the parameter range occupied by the Ta10 sample with our sample, as seen in the various plots provided in this paper.

\subsection{Comparison to the Tr09 sample}

While the Ta10 sample still has a small overlap with the parameter range of our candidates, the Tr09 sample behaves totally differently from our candidate sample in most scaling relations. The Tr09 does not have a single galaxy in common with our sample or the Ta10 sample.

The galaxies of the Tr09 are bluer than almost all our candidates (see Fig. 4), related to their indeed being bluer than the average red sequence galaxies, and some are close to the green valley. This is certainly connected to the younger stellar ages that Trujillo et al. (2009) derived for their sample of 29 "superdense massive galaxies".

The Tr09 sample occupies a different region on the fundamental plane (see Fig. 3) around and beyond the $3-\sigma$ limit, rendering their galaxies outliers on the opposite side to our sample. The galaxies of Tr09 sample have stellar masses comparable to our galaxies (see Fig. 5), but lower dynamical masses (see Fig. 6), which are comparable to those of the Ta10 sample. This and the fact that they have very low mass-to-light ratios (see Figs. 7 and 8) indicate a potential problem. As illustrated in Fig. 9 and more clearly in Fig. 10, the galaxies of the Tr09 sample appear to contain more stellar mass than dynamical mass, which hints at stellar population peculiarities in these objects. The stellar masses that we used have uncertainties of about $40 \%$. We know that these galaxies are very young (about $2 \mathrm{Gyr}$ Trujillo et al. 2009). It seems reasonable to assume that the contrast between the young Tr09 sample and our candidate galaxies may indicate that our sample contains old objects, which are true survivors of the red nuggets from the early universe. 


\subsection{Comparison to other local samples}

Aside from the comparison to the Ta10 and the Tr09 samples, we cross-matched our data with other samples of compact massive early-type galaxies as well. A visual comparison of the location of compact massive early-type galaxies from different authors on the stellar mass-size plane is provided in Fig. 5. We only have the stellar mass of one galaxy in the sample of van den Bosch et al. (2012), namely NGC 1277 , which is located next to the bulk of our sample and b19 in the plot, and it is even one of the denser objects of our sample. None of our candidates has been covered by the HETMGS (van den Bosch et al. 2015). The other samples using galaxies from the local universe (ours, Trujillo et al. 2009; and Taylor et al. 2010) occupy a distinct region in the stellar mass-size plane at the edge or within the zone of exclusion (Burstein et al. 1997; Misgeld \& Hilker 2011; Norris et al. 2014), but with only a small overlap between the individual samples.

As a visual comparison of the different samples in the dynamical mass-size plane, Fig. 6 shows that the samples of Taylor et al. (2010; the Ta10 sample and the one with the parameters from their paper) and Trujillo et al. (2009; the Tr09 sample and the one with the parameters from their paper) are distributed in a large area of the plot partially overlapping. Although small, they are clearly less massive than the galaxies of our sample. The six galaxies of van den Bosch et al. (2012) are within the compact distribution of our candidates in the dynamical mass-size plane, which agrees with our intention to find galaxies similar to them and b19. In Figs. 5 and 6, there may be small systematic deviations between the different samples because the effective radii were measured in different filters. While our sample and the Ta10 samples uses the $r$ band, the sample of Taylor et al. (2010) was measured in the $i$ band, the sample of Trujillo et al. (2009) was measured in the $z$ band and the Tr09 sample uses the $r$ band, and the sample of van den Bosch et al. (2012) was measured in the $K$ band. Our candidates in the redder SDSS bands are smaller than what we measured in the $r$ band by an average of $0.30 \mathrm{kpc}(\sim 14 \%)$ in the $z$ band and $0.11 \mathrm{kpc}(\sim 5 \%)$ in the $i$ band. This suggests that at least the points of the samples of Taylor et al. (2010) and Trujillo et al. (2009) are shifted downwards a little bit in relation to the $r$ band measured points of our sample, the Ta10 sample, and the Tr09 sample in Figs. 5 and 6.

Since b19 was first analysed in Bernardi et al. (2008), we have cross-matched our candidate sample with their list of 43 massive early-type galaxies. Although they also selected their sample by high central velocity dispersion, the only other galaxy in common with our candidate sample, aside from b19, is b17, which has the internal ID 3. However, they do not impose any restriction on the effective radii of their sample, and only a fraction of our sample can match their minimum $\sigma_{0}$ of $350 \mathrm{~km} \mathrm{~s}^{-1}$. Furthermore, the redshifts of most of their galaxies are higher than of the galaxies in our sample, and small galaxies will not be resolved and detected any more at this distance. The sample of Bernardi et al. (2008) is also a subset of SDSS DR1 (Abazajian et al. 2003), which covered a much smaller area of the sky than SDSS DR10, which we used. An interesting result of their paper was that almost half (20 out of 43) of their sample of high $\sigma_{0}$ galaxies are either superposition of two or more galaxies, which is something to consider in the light of follow-up observations.

\subsection{Comparison to intermediate and high redshift samples}

We compared our candidates and the other low redshift samples to various intermediate and high redshift samples. We found quite some difference between them and the local samples, but also between the different high redshift samples themselves. Our candidate sample corresponds best to the subsample of the catalogue of van de Sande et al. (2013) with high dynamical masses.

We used a very recent sample of compact massive galaxies at intermediate redshifts of Zahid et al. (2015), which is based on COSMOS results (Damjanov et al. 2015). Their galaxies are in general more massive, but also less compact than ours. A large number of them are located at the edge of or within the zone of exclusion, as can been seen in comparison to our basic sample in Fig. 5. They seem to form an extension of our sample and the other low redshift sample, but restricted to higher masses and larger radii. As illustrated in Fig. 6, the intermediate redshift sample of Zahid et al. (2015) contains galaxies of similar (and also higher) dynamical masses to our candidates, but they are generally less compact than our galaxies or other low redshift samples. In Fig. 10, the galaxies of Zahid et al. (2015) tend to be more massive than ours and behave like the more massive galaxies of the basic sample. In Fig. 9, they show a tendency to higher central velocity dispersion than does the basic sample, but few of them reach values that are as high as ours.

We used a variety of high redshift samples for comparison, such as the classic high redshift sample of Damjanov et al. (2009), the new high redshift sample of Belli et al. (2014), and the catalogue of van de Sande et al. (2013), which contains a composition of various high redshift samples, such as Bezanson et al. (2013), van Dokkum et al. (2009), Onodera et al. (2012), Cappellari et al. (2009), Newman et al. (2010), van der Wel et al. (2008), Blakeslee et al. (2006), Toft et al. (2012), and their own work. In the size-stellar mass plane (see Fig. 5), all the high redshift samples are distributed along the edge of the zone of exclusion with some objects deeper in to it than any of the low redshift samples. The sample of Belli et al. (2014) consists of more compact and lower mass objects to which the low redshift samples match best. Although the van de Sande et al. (2013) sample is distributed over a wider range of masses, a significant fraction of it shares the same areas with our low redshift samples. The sample of Damjanov et al. (2009) mainly consists of higher mass galaxies, which are on average deeper in the zone of exclusion than the other high redshift samples. Things look differently in the size-dynamical mass plane (see Fig. 6). The galaxies of Belli et al. (2014) take less extreme positions than our candidates in this diagram. However, the Belli et al. (2014) galaxies agree well with the low redshift samples of Taylor et al. (2010) and Trujillo et al. (2009). The sample of van de Sande et al. (2013) contains galaxies with higher dynamical masses and many of its most massive and most compact members agree well with our candidate sample and the sample of van den Bosch et al. (2012).

When analysing Fig. 10, we get a similar result. The sample of Belli et al. (2014) agrees well with Ta10 sample, while a large number of galaxies from the van de Sande et al. (2013) catalogue can be found around our candidates. We have to bear in mind that van de Sande et al. (2013) report a decrease in $M_{*} / M_{\text {dyn }}$ over time, which would explain the shift of our candidates in relation to most of the high redshift sample. In Fig. 9, the high redshift samples are all over the place. There are some tendencies towards higher central velocity dispersions for them, but only a few galaxies (from the van de Sande et al. (2013) sample) posses as high values as our candidates. The distribution of the Sersic indices of the Belli et al. (2014) sample is different from the low redshift sample, since it favours lower values for the Sersic indices as illustrated in Fig. 11. The Sersic indices of the van de Sande et al. (2013) catalogue strongly peak at four, which is the value for de Vaucouleurs profiles. Since the van de Sande et al. (2013) catalogue is a composite of various 
high redshift samples, where not all of them performed a Sersic model fit, but only a de Vaucouleurs fit, this result is not surprising. As illustrated in Fig. 12, the galaxies of the high redshift sample of Belli et al. (2014) are also slightly rounder than our candidates or the Tr09 sample, but not as round as the galaxies of the Ta10 sample.

After this comprehensive analysis, we conclude that the various high redshift samples do not form a very uniform group and that there are differences between the various samples, which raises the question of whether this is due to systematic differences between selection and fitting methods applied to the samples (discussed in van de Sande et al. 2013) or to the red nuggets themselves being a relatively diverse population. Furthermore, none of the low redshift samples agrees in every aspect with the high redshift data. However, one has to keep in mind that ten billion years lie between them, in which the red nuggets may have undergone significant changes.

We explored the connections of our candidate sample to compact galaxies at higher redshifts in Fig. 13 further, where for reasons of comparability, we plot only galaxies whose stellar masses lie between $10^{10.75} M_{\odot}$ and $10^{11.25} M_{\odot}$. It is highlighted in the plot that our candidates are indeed amongst the most compact galaxies of the stellar mass range in SDSS and indeed border the resolution limit of SDSS. We adopt a value of 0.50 arcsec for the observed Vaucouleurs radii in the SDSS $r$ band as resolution limit, which is the lower lower $3 \sigma$-limit of all angular sizes (de Vaucouleurs radii) of early-type galaxies in SDSS. The Ta10 sample and the original values of Taylor et al. (2010) can both be found in the same region of the plot as our candidates, but at slightly lower redshifts. The Trujillo et al. (2009) sample (and naturally the Tr09 sample) appears to be around and partly even beyond the resolution limits of SDSS.

The galaxies of van den Bosch et al. (2012) are much nearer than the galaxies of any other sample and would be beyond the saturation limit of SDSS. The nearest galaxies of our candidate sample can be found close to them. The new intermediate redshift sample of Zahid et al. (2015) mainly contains larger galaxies than the other samples, but their most compact objects are on the expected evolutionary path of the most compact high redshift objects on the way to our candidates. The high redshift samples of Damjanov et al. (2009), Belli et al. (2014), and van de Sande et al. (2013) contain many galaxies that are as compact as the most compact galaxies in the local universe, while containing more mass at the same time. We included the redshift evolution of early-type galaxies of the two highest mass bins from van der Wel et al. (2014b) in our plot. The 16-percentile range of the redshift evolution of an early-type galaxy with a stellar masses of $10^{10.75} M_{\odot}$ (only $16 \%$ of all early-type galaxies at this mass range are more compact than indicated by the line) shows a connection between the two of the most compact galaxies of various high redshift samples, the most compact galaxies of the intermediate redshift sample of Zahid et al. (2015), and our candidates (if extrapolated to the redshift ranges of our sample).

The 16-percentile range of the redshift evolution of an earlytype galaxy with a stellar masses of $10^{11.25} M_{\odot}$ only extends down to a redshift of 0.75 in van der Wel et al. (2014b), because for some unknown reason, the value at a redshift of 0.25 for this percentile and mass is missing in their table for circularized radii, but an educated guess based on the other values and other tables would yield a value of $\log _{10}\left(R_{0}\right) \sim 0.6$. The stellar masses of almost all of our candidates range between $10^{10.75} M_{\odot}$ and $10^{11.25} M_{\odot}$ (we only plotted those within that range in Fig. 13), which means that when comparing with the extrapolation of the redshift evolution of van der Wel et al. (2014b), we found that our candidates are clearly amongst the most compact galaxies of their mass range and that they may be relics of the red nuggets.

\subsection{Space density}

We calculated the space density of candidate sample considering the Malmquist-bias and a resolution limit of 0.50 arcsec (lower $3 \sigma$-limit). The value that we obtained this way is $4 \times$ $10^{-7}$ galaxies $/ \mathrm{Mpc}^{3}$, which is about 400 times lower than the space density of red nuggets at $z=2$ (Quilis \& Trujillo 2013). However, when comparing space densities of different samples, one has to be aware of the selection criteria used to define them and biases affecting them. Our sample only contains one galaxy above a redshift of 0.2 , while the rest are clearly below it. This is a consequence of the selection bias from using spectroscopic data from SDSS. Galaxies with very small angular sizes (below the resolution limit mentioned above) are not included in the basic sample, which explains the dearth of compact galaxies at higher redshifts in our sample. Most samples (Trujillo et al. 2009; Taylor et al. 2010; Quilis \& Trujillo 2013) use stellar masses as a selection criterion. We tried to derive more comparable quantities by considering additional selection criteria for our candidate galaxies, which are similar to the selection criteria of other authors. We restricted our candidate sample to dynamical masses greater than $8 \times 10^{10} M_{\odot}$ and physical radii of less than $2 \mathrm{kpc}$ to be better comparable with the predication for old (formed before a redshift of 2) compact massive galaxies by Damjanov et al. (2014). Fifty-eight of 76 galaxies in the candidate sample fulfil this condition, and they obtain a space density of $3.6 \times 10^{-7}$ galaxies $/ \mathrm{Mpc}^{3}$ for our sample, which is located at redshifts below 0.2 . The lowest redshift interval considered in Damjanov et al. (2014) is 0.2 to 0.3 , and they predict a space density of $2.6_{2.4}^{1.2} \times 10^{-7}$ galaxies $/ \mathrm{Mpc}^{3}$, which is about seven times higher than ours.

For our comparison with the space density of Trujillo et al. (2009), we applied the same restriction as they do on our candidate sample. The stellar masses have to be higher than $8 \times$ $10^{10} M_{\odot}$, and the $z$ band physical radii smaller than $1.5 \mathrm{kpc}$. This reduces our candidate sample to merely 16 galaxies, and we obtained a space density for them of $5.9 \times 10^{-8}$ galaxies $/ \mathrm{Mpc}^{3}$, which is by more than a factor of two lower than the upper limit of the space density of Trujillo et al. (2009), which is $1.3 \times 10^{-7}$ galaxies/ $\mathrm{Mpc}^{3}$ (Quilis \& Trujillo 2013). Comparing to space densities to the Taylor et al. (2010) is more difficult due to their definition. We only considered their restriction that the stellar masses have to be higher than $10^{10.7} M_{\odot}$. Hence the space density, which we derived using a subsample of 67 galaxies of our candidates, is an upper limit. We obtained a value of $2.7 \times 10^{-7}$ galaxies/ $\mathrm{Mpc}^{3}$, which is almost an order of magnitude higher than the value of Taylor et al. (2010) of $3 \times 10^{-8}$ galaxies/Mpc ${ }^{3}$ (Quilis \& Trujillo 2013). We thus found that the space densities we derived are comparable within an order of magnitude to those of other samples in the local (and intermediate redshift) universe, but we refrain from a strong interpretation of our numbers compared to the high redshift universe given the dominating influence of selection effects on the results.

\subsection{Individual galaxies}

Although the candidate galaxies form a very homogeneous group in all their properties, one can identify particularly peculiar objects by focusing on some individual galaxies. There are seven galaxies in our sample with radii less than a kpc. The smallest one has the internal ID 63 and a physical radius 


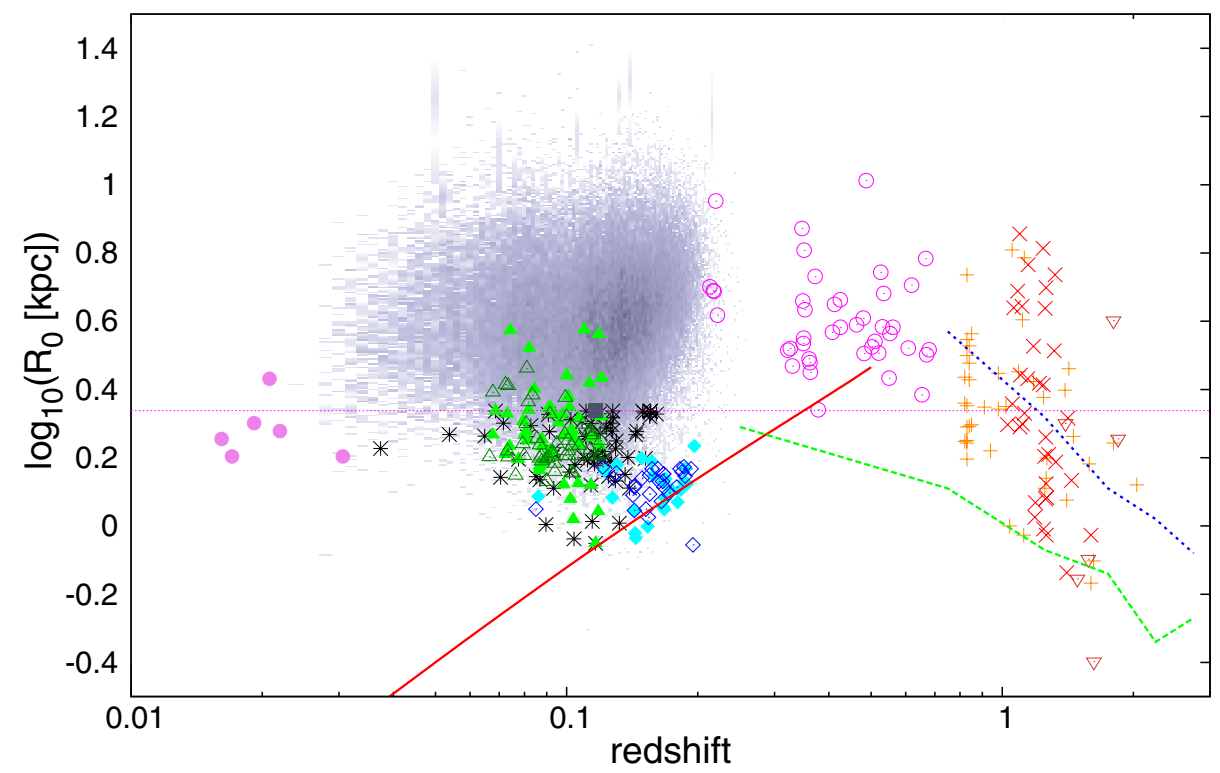

candidates (this paper) *

Ta10 sample $\Delta$

Tr09 sample

Taylor+ $2010 \triangle$

Trujillo+ 2009

van de Sande+ 2013

Belli+ 2014

Damjanov+ 2009

Zahid+ 2015

van den Bosch+ 2012

b19 (this paper)

SDSS size limit

size evolution lower mass

size evolution high mass ….....

Fig. 13. Redshift vs. size distribution for galaxies of different samples within a stellar mass range between $10^{10.75} M_{\odot}$ and $10^{11.25} M_{\odot}$. The blueish cloud in the background represents all galaxies of the basic sample with stellar masses greater than $10^{10.75} M_{\odot}$ and less than $10^{11.25} M_{\odot}$. The black stars represent the candidates of our sample. The galaxies of the Ta10 sample are shown using filled green triangles and the galaxies of the Tr09 sample are indicated by filled cyan diamonds. The galaxies from Taylor et al. (2010) using the values of their paper are indicated by open dark green triangles. The open blue diamonds represent the galaxies of Trujillo et al. (2009). Orange crosses denote the catalogue of various high redshift samples by van de Sande et al. (2013). The high redshift sample of Belli et al. (2014) is indicated by red Xs. Open brown nabla symbols indicate the high redshift galaxies of Damjanov et al. (2009). Open magenta circles indicate the intermediate redshift sample of Zahid et al. (2015). The six galaxies of van den Bosch et al. (2012) are represented by filled violet circles. Using our calibration of SDSS data, b19 is shown by a filled grey square. The magenta dashed line denotes the limiting scaling radius for our sample selection. The red solid line corresponds to the angular resolution limit of SDSS of 0.50 arcsec. The green dashed line denotes the 16-percentile range of the redshift evolution of an early-type galaxy with a stellar masses of $10^{10.75} M_{\odot}$ according to Table A1 of van der Wel et al. (2014b) and the blue dotted line indicates the 16-percentile range of the redshift evolution of an early-type galaxy with a stellar masses of $10^{11.25} M_{\odot}$ based on the same work.

of $R_{0}=(0.62 \pm 0.01) \mathrm{kpc}$. At its redshift of 0.0877 , this means that the observed angular semi-major axis $a_{\text {sdss }}$ is only $(0.47 \pm$ $0.01)$ arcsec and therefore at the limit of SDSS resolution. Galaxy 63 also happens to be the second faintest object of our sample, with an $r$ band absolute magnitude of $(-20.51 \pm$ $0.01)$, and it has the lowest surface brightness with $(17.12 \pm$ $0.05) \mathrm{mag} / \operatorname{arcsec}^{2}$ in the $r$ band. The galaxy also stands out by its mass: it possesses the lowest dynamical mass of our candidates with $M_{\text {dyn }}=(8.13 \pm 0.38) \times 10^{10} M_{\odot}$ and the second lowest stellar mass of our sample with $M_{*}=(2.82 \pm 1.16) \times 10^{10} M_{\odot}$. By being a peculiarly compact and faint, but also relatively low mass object in our sample, we consider galaxy 63 as one of the most interesting objects for our follow-up observations.

The other extremely small objects in our sample show similar properties to galaxy 63 . The faintest object with an absolute $r$ band magnitude of only $(-20.35 \pm 0.02)$ is, with a physical radius of $R_{0}=(0.78 \pm 0.02) \mathrm{kpc}$, also the third smallest galaxy in the candidate sample. It has an internal ID of 31 and is located at a redshift of 0.0784 . Galaxy 31 is with an observed angular semi-major axis $a_{\text {sdss }}$ of only $(0.64 \pm 0.02)$ arcsec one of the galaxies close to the resolution limit of SDSS. Although this galaxy has the lowest stellar mass of our candidate sample with $M_{*}=(2.57 \pm 1.06) \times 10^{10} M_{\odot}$, its dynamical mass $M_{\mathrm{dyn}}=$ $(1.25 \pm 0.06) \times 10^{11} M_{\odot}$ is fairly average for our candidate sample. In combination with the fact that it is the faintest galaxy, this results in a relatively high dynamical mass-to-light ratio of $\Upsilon_{\text {dyn }}=(11.99 \pm 0.82) M_{\odot} / L_{\odot, r}$. This makes it a promising object for follow-up observations.

At the other extreme of the angular sizes, we have our three best-resolved galaxies with $a_{\text {sdss }}$ greater than 3 arcsec. The galaxy with an internal ID of 75 is with an angular semi-major axis of $(3.59 \pm 0.02)$ arcsec not only the apparently biggest object in the sky of our candidate sample, but also the nearest. It is located at a redshift of 0.0260 . Galaxy 75 is also outstanding because it has the by far highest dynamical mass-to-light ratio of our sample with $\Upsilon_{\text {dyn }}=(20.56 \pm 2.13) M_{\odot} / L_{\odot, r}$ and, with 0.51 (which is just over the selection criterion of 0.50 ), the lowest GalaxyZoo probability $\mathcal{L}_{\text {ETG }}$ for a galaxy to be classified as an early type within our sample. A visual inspection of the galaxy (see Fig. 2) shows a face-on featureless disc, which differs from the other candidate galaxies. A manual analysis of the SDSS classification of its spectrum confirms our suspicion that this galaxy is no regular early-type galaxy, but rather a starburst galaxy. We therefore consider it a false positive in our sample.

The next largest galaxy in angular size has the internal ID 50, which also happens to be, with a redshift of 0.0374 , the second nearest candidate galaxy. It possesses a physical radius of $R_{0}=$ $(1.69 \pm 0.01) \mathrm{kpc}$ and an absolute $r$ band magnitude of $(-21.56 \pm$ $0.01)$. With a dynamical mass of $M_{\text {dyn }}=(2.24 \pm 0.05) \times 10^{11} M_{\odot}$, a stellar mass of $M_{*}=(8.13 \pm 3.35) \times 10^{10} M_{\odot}$, and no outstanding mass-to-light ratios, galaxy 50 is an average example of our candidates. Its low redshift allows for easier follow-up observations of this object, so we rank it as one of our priority candidates.

The brightest galaxy in our sample has the internal ID 66 and shines with an absolute magnitude of $(-22.69 \pm 0.01)$ mag in the $r$ band. It is located at a redshift of $0.2018^{6}$ and is therefore

\footnotetext{
6 It is also the only galaxy beyond a redshift of 0.2 in our candidate sample.
} 
the most distant galaxy of our candidates. It has a physical radius of $R_{0}=(2.06 \pm 0.08) \mathrm{kpc}$ and is one of the largest candidates. Galaxy 66 possesses a dynamical mass of $M_{\text {dyn }}=$ $(2.75 \pm 0.20) \times 10^{11} M_{\odot}$, and it stands out with the second highest stellar mass of our candidates with $M_{*}=(1.86 \pm 0.77) \times$ $10^{11} M_{\odot}$. Another outstanding property of galaxy 66 is its dynamical mass-to-light ratio of $\Upsilon_{\text {dyn }}=(3.03 \pm 0.19) M_{\odot} / L_{\odot, r}$ is the lowest of our candidate sample, and we found that is also has the fifth lowest stellar mass-to-light ratio with $\Upsilon_{*}=$ $(2.05 \pm 0.84) M_{\odot} / L_{\odot, r}$. We consider galaxy 66 a priority candidate for follow-up observations, although its high redshift will make them more difficult, but on the other hand the high luminosity of the galaxy will help a bit.

The galaxy with the highest stellar mass has the internal ID 56. It has a stellar mass of $M_{*}=(1.91 \pm 0.79) \times 10^{11} M_{\odot}$ and a dynamical mass of $M_{\text {dyn }}=(2.95 \pm 0.28) \times 10^{11} M_{\odot}$. The galaxy with a physical radius of $R_{0}=(2.00 \pm 0.14) \mathrm{kpc}$ is also the second most distant candidate with a redshift of 0.1978 . It is the second reddest object with a g-r colour of $(1.04 \pm 0.03)$ mag, which makes it one of only two outliers of our candidate sample above the upper $3-\sigma$ limit of the red sequence. Galaxy 56 also stands out for having the highest stellar mass-to-light ratio of our candidate sample with $\Upsilon_{*}=(4.27 \pm 1.77) M_{\odot} / L_{\odot, r}$. This and its average dynamical mass-to-light ratio of $\Upsilon_{\text {dyn }}=$ $(6.67 \pm 0.71) M_{\odot} / L_{\odot, r}$ contrasts it with the previously discussed galaxy 66. Galaxy 56 is a very interesting object and qualifies as priority target for our follow-up observations.

The galaxy with the highest dynamical mass has the internal ID 23. It contains a stellar mass of $M_{*}=(1.10 \pm 0.45) \times 10^{11} M_{\odot}$ and a dynamical mass of $M_{\text {dyn }}=(4.57 \pm 0.22) \times 10^{11} M_{\odot}$. This is due to its high central velocity dispersion of $\sigma_{0}=(423 \pm$ 17) $\mathrm{km} \mathrm{s}^{-1}$ and its relatively large physical radius $R_{0}=(2.17 \pm$ $0.04) \mathrm{kpc}$, which is close to the limit of our sample selection. Galaxy 23 has the fourth highest dynamical mass-to-light ratio with $\Upsilon_{\text {dyn }}=(12.70 \pm 0.64) M_{\odot} / L_{\odot, r}$ and an average stellar massto-light ratio of $\Upsilon_{*}=(2.96 \pm 1.22) M_{\odot} / L_{\odot, r}$.

Aside from the troublesome galaxy 75 , galaxy 30 has the highest dynamical mass-to-light ratio with $\Upsilon_{\text {dyn }}=(13.21 \pm$ $0.53) \times M_{\odot} / L_{\odot, r}$. In contrast to this, its stellar mass-to-light ratio is one of the lowest with only $\Upsilon_{*}=(1.99 \pm 0.82) M_{\odot} / L_{\odot, r}$. This agrees with Galaxy 30 , which has the third lowest stellar mass of the candidate sample with $M_{*}=(3.72 \pm 1.53) \times 10^{10} M_{\odot}$. Galaxy 30 has a dynamical mass of $M_{\text {dyn }}=(2.45 \pm 0.12) \times$ $10^{11} M_{\odot}$. It is worth pointing out that it has neither an extraordinarily high central velocity dispersion of $\sigma_{0}=(346 \pm 11) \mathrm{km} \mathrm{s}^{-1}$ nor a large physical radius $R_{0}=(1.76 \pm 0.03) \mathrm{kpc}$ for the candidate galaxies.

The galaxy with the highest central velocity dispersion is galaxy 76 with $\sigma_{0}=(432 \pm 18) \mathrm{km} \mathrm{s}^{-1}$. With an absolute $r$ band magnitude of $(-22.07 \pm 0.01)$, this galaxy is one of the brighter objects in our candidate sample. It is also the second most massive galaxy in our sample in terms of dynamical mass with $M_{\text {dyn }}=(4.47 \pm 0.21) \times 10^{11} M_{\odot}$.

The reddest galaxy in our candidate sample has the internal ID 12 . With a $g-r$ colour of $(-1.10 \pm 0.02) \mathrm{mag}$, it is a clear outlier on the red sequence and significantly redder than all galaxies in our candidate sample (except one) and even than most galaxies in the basic sample (see Fig. 4). Surprisingly, galaxy 12 is not outstanding in any other parameters than colour, and it appears to be an average member of the candidate sample.

We visually inspected the images of all our candidates (see Fig. 2) and find that several have other (foreground or background) objects that are only less than five angular scale radii $a_{\text {sdss }}$ from their centre. The galaxies with the internal IDs 18, 24, 25, 33, 35, and 50 have other prominent objects (galaxies or stars) within their immediate vicinity. We have to be careful when using the parameters measured for these galaxy, since there is a chance that their values might suffer from some contamination.

Last but not least, we return to the starting point of our investigation, b19, which has the internal ID 2. It is a member of our candidate sample with rather average properties compared to the other 75 candidates. It is always a central part of the group of data points formed by our candidates in the diagrams. The only feature of b19 that is a little outstanding compared to the other members of the candidate sample is its Sersic parameter $n_{\mathrm{S}}$, which borders the maximum allowed value of eight by the algorithm used by Simard et al. (2011). This is close to the Sersic index of 6.9 found by Läsker et al. (2013) based on HST/ACS/HRC imaging.

\section{Summary and conclusions}

Our sample of 76 candidates (including b19) for compact massive early-type galaxies forms an ideal basis for future follow-up observations using high-resolution spectroscopy and imaging. We found that our homogeneous sample, which has been defined as extreme outliers in the $\log _{10}\left(R_{0}\right)-\log _{10}\left(\sigma_{0}\right)$ plane does not behave as do outliers in other relations for early-type galaxies except for those that are directly related to the selection criteria. The candidates seem to form the compact massive tail of the general distribution of early-type galaxies, and are not a separate population of particularly peculiar objects. We confirmed that there is an increase in the dynamical and stellar mass-tolight ratios at higher central velocity dispersions.

Furthermore, we saw the same tendency as Conroy et al. (2013) that the difference between the dynamical mass and the stellar mass derived using simple models (Mendel et al. 2014) increases at higher central velocity dispersions. This is usually considered to be an indication of a systematic variation in the initial mass function of those galaxies. As a result our candidates may have an extremely bottom-heavy initial mass function as proposed for b19 (Läsker et al. 2013). The high central velocity dispersion, which is crucial for determining of the dynamical mass, could also be due to over-massive central black holes (van den Bosch et al. 2012; Mieske et al. 2013; Seth et al. 2014). However, a robust detection of such a black hole is only possible for the nearest objects with high spatial resolution spectroscopy (Läsker et al. 2013; Emsellem 2013; Y1ldırım et al., submitted). It is unlikely that all these objects contain such a large black hole.

We also tried to draw a connection between the compact massive galaxies in our sample and even more massive and more compact galaxies from high redshifts. Based on the previous observation of quiescent high-redshift galaxies (Kriek et al. 2006, 2008), van Dokkum et al. (2008) found that there are already fully formed early-type galaxies with scale radii that are much smaller and stellar masses that are much higher than any object known in the local universe. For comparison, we used several samples of these galaxies at high redshifts, such as those of Damjanov et al. (2009), van de Sande et al. (2013), and Belli et al. (2014), and also at intermediate redshifts as in Zahid et al. (2015). Many of these galaxies must have evolved, most likely by mergers, into more regular early-type galaxies. The galaxies in our sample are those that still resemble to a specific subgroup of these red nuggets. Some of these exotic galaxies in the early universe possess high dynamical masses and small sizes. 
From some local galaxies, such as b19 (Läsker et al. 2013) and NGC 1277, and others (van den Bosch et al. 2012), we know similar features, and they are remnants of red nugget galaxies. It is shown in van de Sande et al. (2015) that although the initial mass function of most red nuggets shows a rather shallow slope, the one for those galaxies with high mass-to-light ratios tend to be bottom-heavy. The galaxy b19 is known for its bottom heavy initial mass function and our candidates have an elevated mass-to-light ratio in comparison to normal early-type galaxies. Therefore, one may suspect that some galaxies of our sample are remnants of these ancient objects or that they are at least somehow related to the subgroup of these objects where the galaxies of Läsker et al. (2013) and van den Bosch et al. (2012) originated. The advantage of our sample is that it is located in the local universe and is thus easier to study than galaxies at high redshifts.

By selecting galaxies based on stellar velocity dispersion, we found 76 compact, massive early-type galaxies below a redshift of $z=0.2018$. These are excellent targets for further studies of various scientific questions, such as the variation in the initial mass function, over-massive black holes, and potential remnants of exotic galaxies from the early universe. High-resolution imaging data can confirm their sizes and rule out superpositions. Spectroscopy of these objects will enable us to study their dynamical mass distribution and kinematics, and it will allow for (resolved) stellar population analysis.

Acknowledgements. Funding for SDSS-III has been provided by the Alfred P. Sloan Foundation, the Participating Institutions, the National Science Foundation, and the US Department of Energy Office of Science. The SDSSIII web site is http://www.sdss3.org/. SDSS-III is managed by the Astrophysical Research Consortium for the Participating Institutions of the SDSS-III Collaboration including the University of Arizona, the Brazilian Participation Group, Brookhaven National Laboratory, University of Cambridge, Carnegie Mellon University, University of Florida, the French Participation Group, the German Participation Group, Harvard University, the Instituto de Astrofisica de Canarias, the Michigan State/Notre Dame/JINA Participation Group, Johns Hopkins University, Lawrence Berkeley National Laboratory, Max Planck Institute for Astrophysics, Max Planck Institute for Extraterrestrial Physics, New Mexico State University, New York University, Ohio State University, Pennsylvania State University, University of Portsmouth, Princeton University, the Spanish Participation Group, University of Tokyo, University of Utah, Vanderbilt University, University of Virginia, University of Washington, and Yale University. C.S. acknowledges the support from an ESO studentship. C.S. acknowledges support from the MPIA for hosting him in Heidelberg for a couple of days. C.S. acknowledges helpful advise from Jens-Kristian Krogager and fruitful discussions with Ronald Läsker and Karina Voggel.

\section{References}

Abazajian, K., Adelman-McCarthy, J. K., Agüeros, M. A., et al. 2003, AJ, 126, 2081

Abazajian, K. N., Adelman-McCarthy, J. K., Agüeros, M. A., et al. 2009, ApJS, 182,543

Ahn, C. P., Alexandroff, R., Allende Prieto, C., et al. 2014, ApJS, 211, 17

Aihara, H., Allende Prieto, C., An, D., et al. 2011, ApJS, 193, 29

Belli, S., Newman, A. B., \& Ellis, R. S. 2014, ApJ, 783, 117

Bernardi, M., Sheth, R. K., Annis, J., et al. 2003, AJ, 125, 1866

Bernardi, M., Hyde, J. B., Fritz, A., et al. 2008, MNRAS, 391, 1191

Bertin, G., Ciotti, L., \& Del Principe, M. 2002, A\&A, 386, 149

Bezanson, R., van Dokkum, P., van de Sande, J., Franx, M., \& Kriek, M. 2013, ApJ, 764, L8

Blakeslee, J. P., Holden, B. P., Franx, M., et al. 2006, ApJ, 644, 30

Blanton, M. R., \& Roweis, S. 2007, AJ, 133, 734

Blanton, M. R., Schlegel, D. J., Strauss, M. A., et al. 2005, AJ, 129, 2562

Burstein, D., Bender, R., Faber, S., \& Nolthenius, R. 1997, AJ, 114, 1365
Cappellari, M., Bacon, R., Bureau, M., et al. 2006, MNRAS, 366, 1126 Cappellari, M., Emsellem, E., Bacon, R., et al. 2007, MNRAS, 379, 418 Cappellari, M., di Serego Alighieri, S., Cimatti, A., et al. 2009, ApJ, 704, L34 Chevance, M., Weijmans, A.-M., Damjanov, I., et al. 2012, ApJ, 754, L24 Chilingarian, I. V., \& Zolotukhin, I. Y. 2012, MNRAS, 419, 1727

Chilingarian, I. V., Melchior, A., \& Zolotukhin, I. Y. 2010, MNRAS, 405, 1409 Conroy, C., Gunn, J. E., \& White, M. 2009, ApJ, 699, 486

Conroy, C., Dutton, A. A., Graves, G. J., Mendel, J. T., \& van Dokkum, P. G. 2013, ApJ, 776, L26

Damjanov, I., McCarthy, P. J., Abraham, R. G., et al. 2009, ApJ, 695, 101

Damjanov, I., Hwang, H. S., Geller, M. J., \& Chilingarian, I. 2014, ApJ, 793, 39

Damjanov, I., Geller, M. J., Zahid, H. J., \& Hwang, H. S. 2015, ApJ, submitted [arXiv: 1501.04976]

Djorgovski, S., \& Davis, M. 1987, ApJ, 313, 59

Dressler, A., Lynden-Bell, D., Burstein, D., et al. 1987, ApJ, 313, 42

Emsellem, E. 2013, MNRAS, 433, 1862

Emsellem, E., Cappellari, M., Krajnović, D., et al. 2007, MNRAS, 379, 401

Hyde, J. B., \& Bernardi, M. 2009, MNRAS, 396, 1171

Jorgensen, I., Franx, M., \& Kjaergaard, P. 1995, MNRAS, 276, 1341

Kauffmann, G., Heckman, T. M., White, S. D. M., et al. 2003, MNRAS, 341, 33

Krajnović, D., Bacon, R., Cappellari, M., et al. 2008, MNRAS, 390, 93

Kriek, M., van Dokkum, P. G., Franx, M., et al. 2006, ApJ, 649, L71

Kriek, M., van Dokkum, P. G., Franx, M., et al. 2008, ApJ, 677, 219

La Barbera, F., de Carvalho, R. R., de La Rosa, I. G., \& Lopes, P. A. A. 2010, MNRAS, 408, 1335

Läsker, R., van den Bosch, R. C. E., van de Ven, G., et al. 2013, MNRAS, 434, L31

Lintott, C. J., Schawinski, K., Slosar, A., et al. 2008, MNRAS, 389, 1179

Lintott, C., Schawinski, K., Bamford, S., et al. 2011, MNRAS, 410, 166

Mendel, J. T., Simard, L., Palmer, M., Ellison, S. L., \& Patton, D. R. 2014, ApJS, 210, 3

Mieske, S., Frank, M. J., Baumgardt, H., et al. 2013, A\&A, 558, A14

Misgeld, I., \& Hilker, M. 2011, MNRAS, 414, 3699

Newman, A. B., Ellis, R. S., Treu, T., \& Bundy, K. 2010, ApJ, 717, L103

Norris, M. A., Kannappan, S. J., Forbes, D. A., et al. 2014, MNRAS, 443, 1151

Onodera, M., Renzini, A., Carollo, M., et al. 2012, ApJ, 755, 26

Poggianti, B. M., Moretti, A., Calvi, R., et al. 2013, ApJ, 777, 125

Quilis, V., \& Trujillo, I. 2013, ApJ, 773, L8

Salim, S., Rich, R. M., Charlot, S., et al. 2007, ApJS, 173, 267

Saulder, C., Mieske, S., Zeilinger, W. W., \& Chilingarian, I. 2013, A\&A, 557, A21

Schlegel, D. J., Finkbeiner, D. P., \& Davis, M. 1998, ApJ, 500, 525

Seth, A. C., van den Bosch, R., Mieske, S., et al. 2014, Nature, 513, 398

Simard, L., Mendel, J. T., Patton, D. R., Ellison, S. L., \& McConnachie, A. W. 2011, ApJS, 196, 11

Strauss, M. A., Weinberg, D. H., Lupton, R. H., et al. 2002, AJ, 124, 1810

Taylor, E. N., Franx, M., Glazebrook, K., et al. 2010, ApJ, 720, 723

Terlevich, R., Davies, R. L., Faber, S. M., \& Burstein, D. 1981, MNRAS, 196, 381

Toft, S., Gallazzi, A., Zirm, A., et al. 2012, ApJ, 754, 3

Trujillo, I., Feulner, G., Goranova, Y., et al. 2006, MNRAS, 373, L36

Trujillo, I., Cenarro, A. J., de Lorenzo-Cáceres, A., et al. 2009, ApJ, 692, L118

Trujillo, I., Ferré-Mateu, A., Balcells, M., Vazdekis, A., \& Sánchez-Blázquez, P. 2014, ApJ, 780, L20

Valentinuzzi, T., Fritz, J., Poggianti, B. M., et al. 2010, ApJ, 712, 226

van de Sande, J., Kriek, M., Franx, M., et al. 2013, ApJ, 771, 85

van de Sande, J., Kriek, M., Franx, M., Bezanson, R., \& van Dokkum, P. G. 2015, ApJ, 799, 125

van den Bosch, R. C. E., Gebhardt, K., Gültekin, K., et al. 2012, Nature, 491, 729

van den Bosch, R., Gebhardt, K., Gültekin, K., Yıldırım, A., \& Walsh, J. 2015, ApJS, 218, 10

van der Wel, A., Holden, B. P., Zirm, A. W., et al. 2008, ApJ, 688, 48

van der Wel, A., Rix, H.-W., Wuyts, S., et al. 2011, ApJ, 730, 38

van der Wel, A., Chang, Y.-Y., Bell, E. F., et al. 2014a, ApJ, 792, L6

van der Wel, A., Franx, M., van Dokkum, P. G., et al. 2014b, ApJ, 788, 28

van Dokkum, P. G., Franx, M., Kriek, M., et al. 2008, ApJ, 677, L5

van Dokkum, P. G., Kriek, M., \& Franx, M. 2009, Nature, 460, 717

Wegner, G., Colless, M., Saglia, R. P., et al. 1999, MNRAS, 305, 259

Yıldırım, A., van den Bosch, R. C. E., Gebhardt, K., et al. 2014, MNRAS, submitted

Zahid, H. J., Damjanov, I., Geller, M., \& Chilingarian, I. 2015, ArXiv e-prints [arXiv: 1501.04977] 
C. Saulder et al.: Dozens of compact and high velocity-dispersion, early-type galaxies in Sloan Digital Sky Survey

导言商

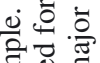
焉 is

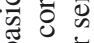
궁 缡 घี ส

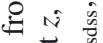

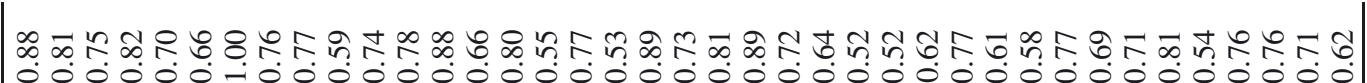
矛

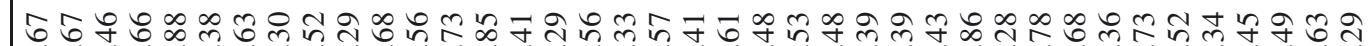
ڤั 000000000000000000000000000000000000000

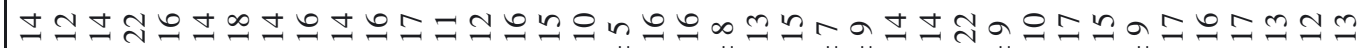
$+1+1+1+1+1+1+1+1+1+1+1+1+1+1+1+1+1+1+1+1+1+1+1+1+1+1+1+1+1+1+1+1+1+1+1+1+1+1$

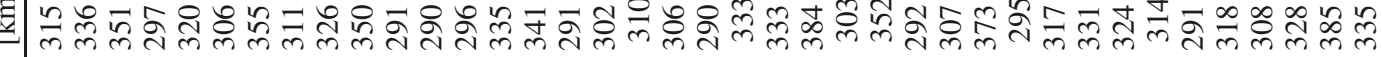
สํㅇำช

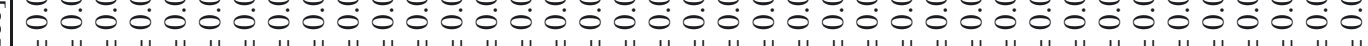

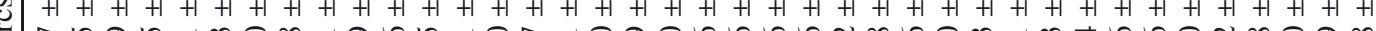
구 ले

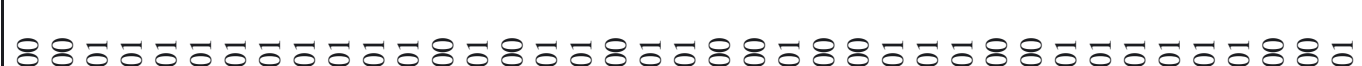

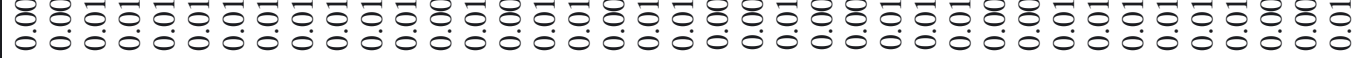

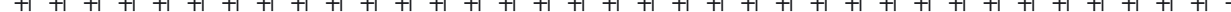

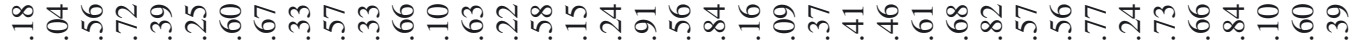

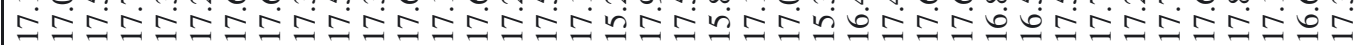

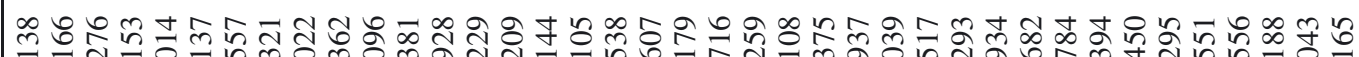

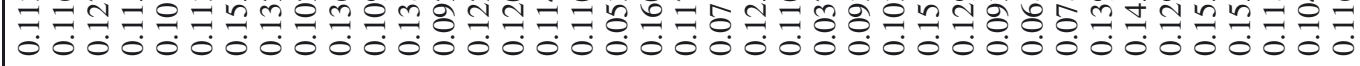
สำ 1 수의 i १守

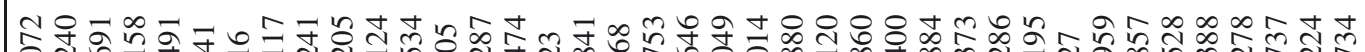
$\infty$ t no m है

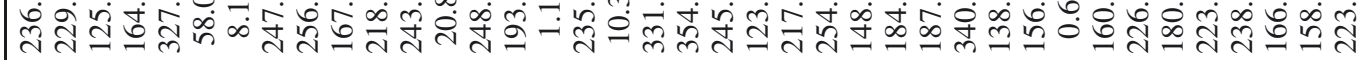

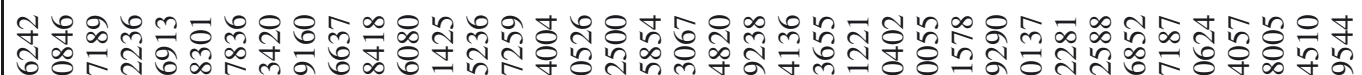

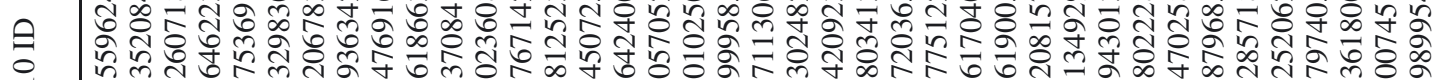

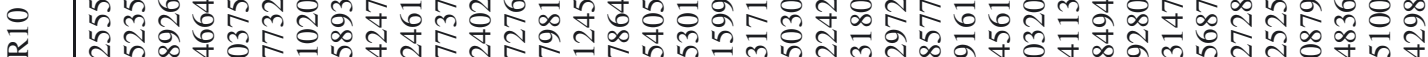




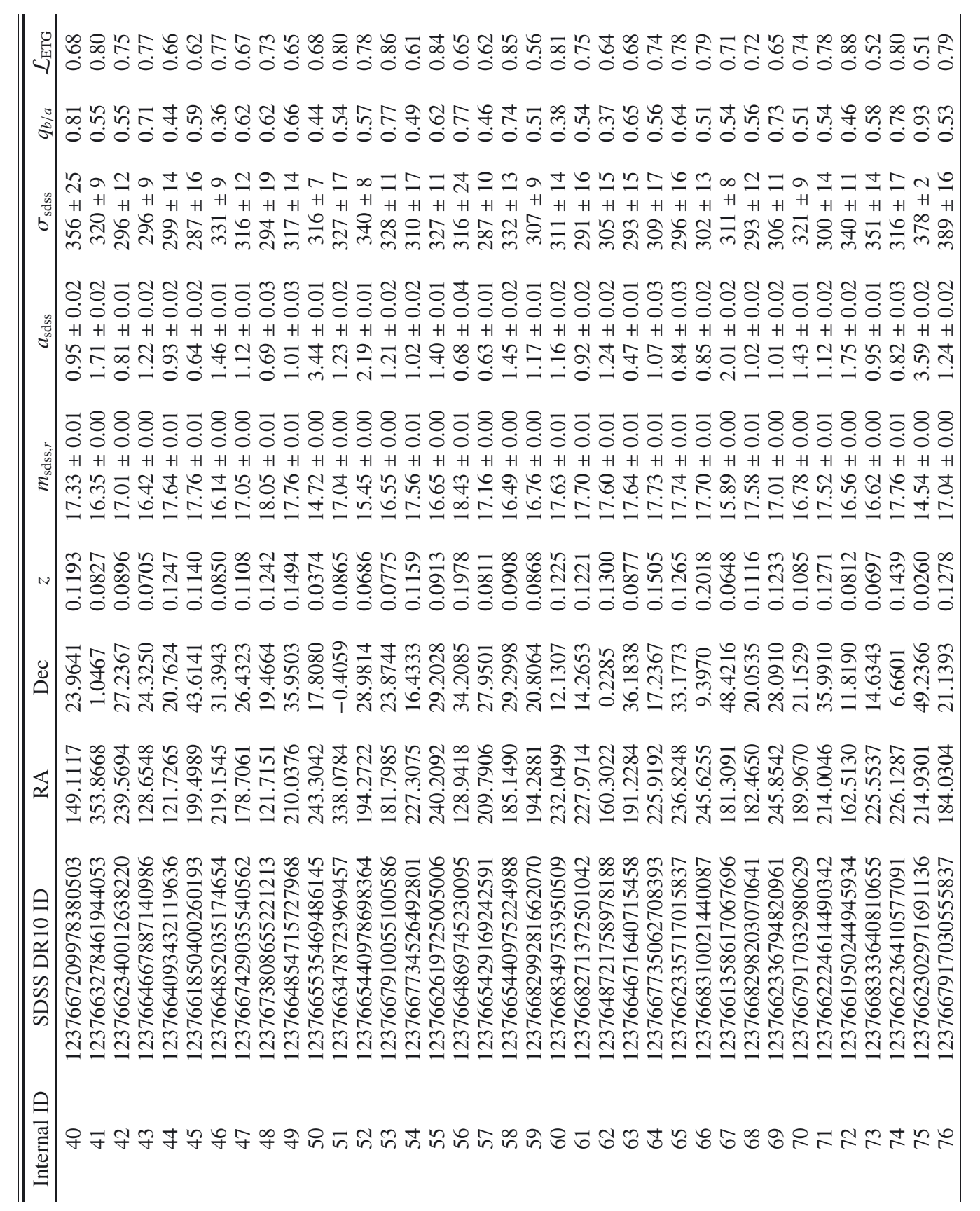




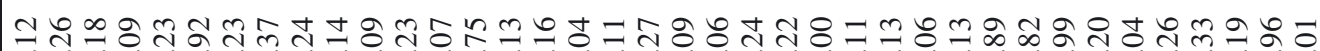

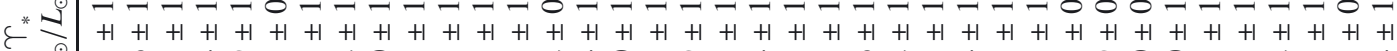

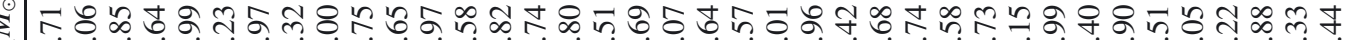

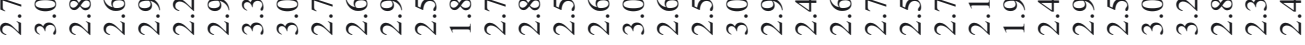

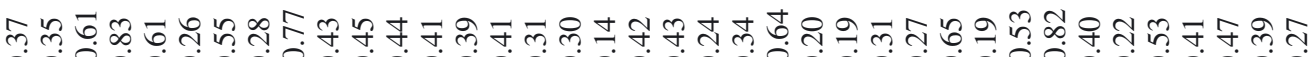

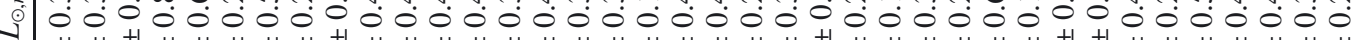

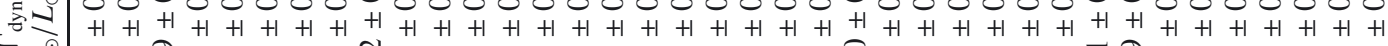

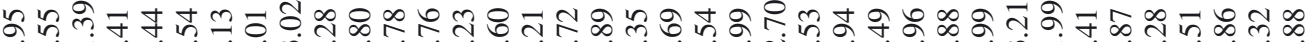

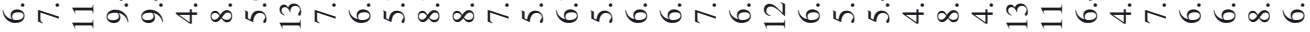

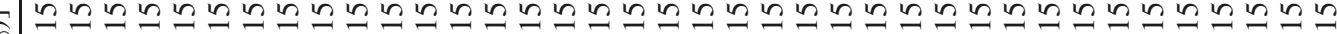
₹ (1) $+1+1+1+1+1+1+1+1+1+1+1+1+1+1+1+1+1+1+1+1+1+1+1+1+1+1+1+1+1+1+1+1+1+1+1+1+1$ 士n

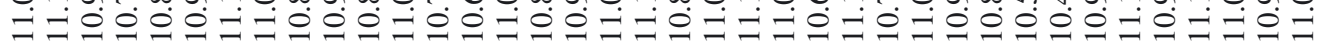

สํㅇำ

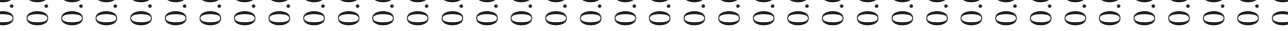
$+1+1+1+1+1+1+1+1+1+1+1+1+1+1+1+1+1+1+1+1+1+1+1+1+1+1+1+1+1+1+1+1+1+1+1+1+1+1$ ケ

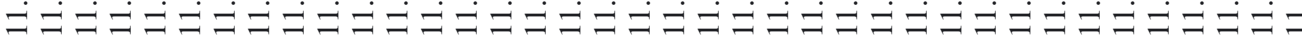

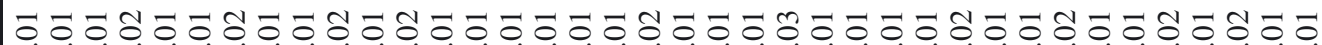

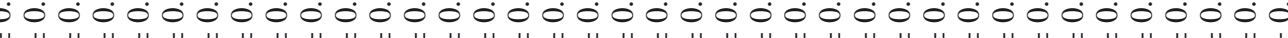
4ง

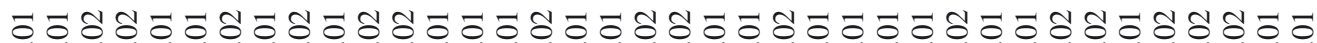

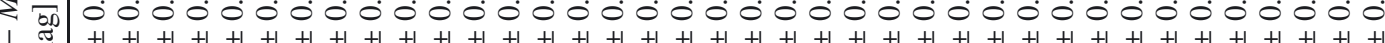
$2+1+1+1+1+1+1+1+1+1+1+1+1+1+1+1+1+1+1+1+1+1+1+1+1+1+1+1+1+1+1+1+1+1+1+1+1+1$

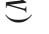

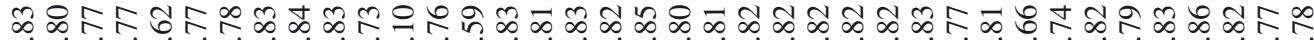

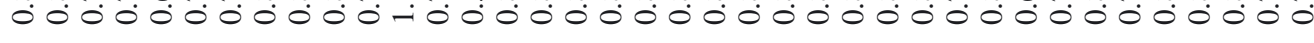

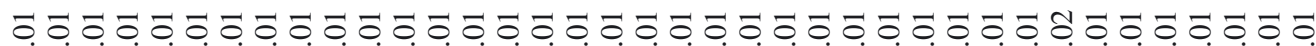

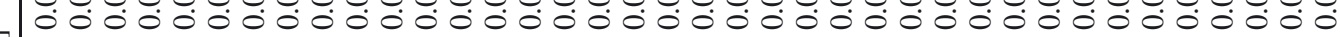
$+1+1+1+1+1+1+1+1+1+1+1+1+1+1+1+1+1+1+1+1+1+1+1+1+1+1+1+1+1+1+1+1+1+1+1+1+1+1$

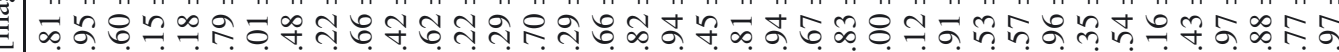

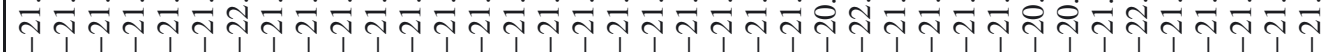

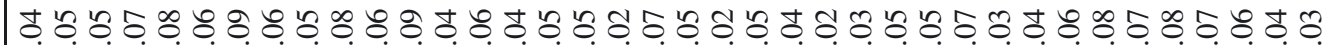

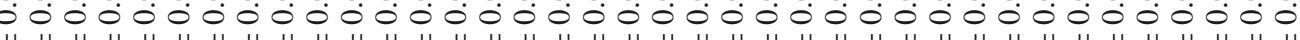
m

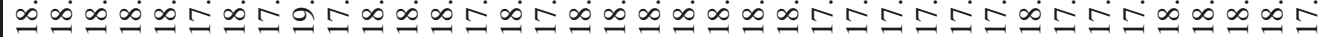

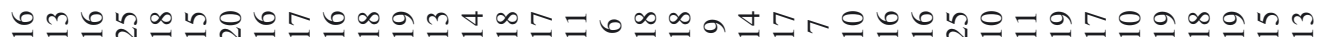
$+1+1+1+1+1+1+1+1+1+1+1+1+1+1+1+1+1+1+1+1+1+1+1+1+1+1+1+1+1+1+1+1+1+1+1+1+1+1$

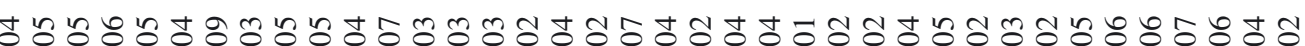

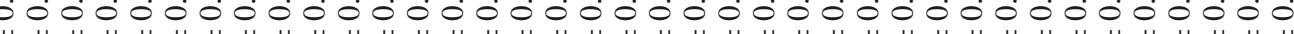

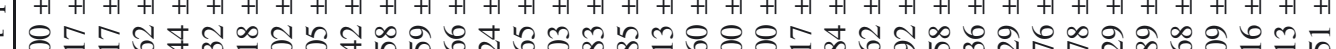
idi-i

目

是

$\|$ 西 


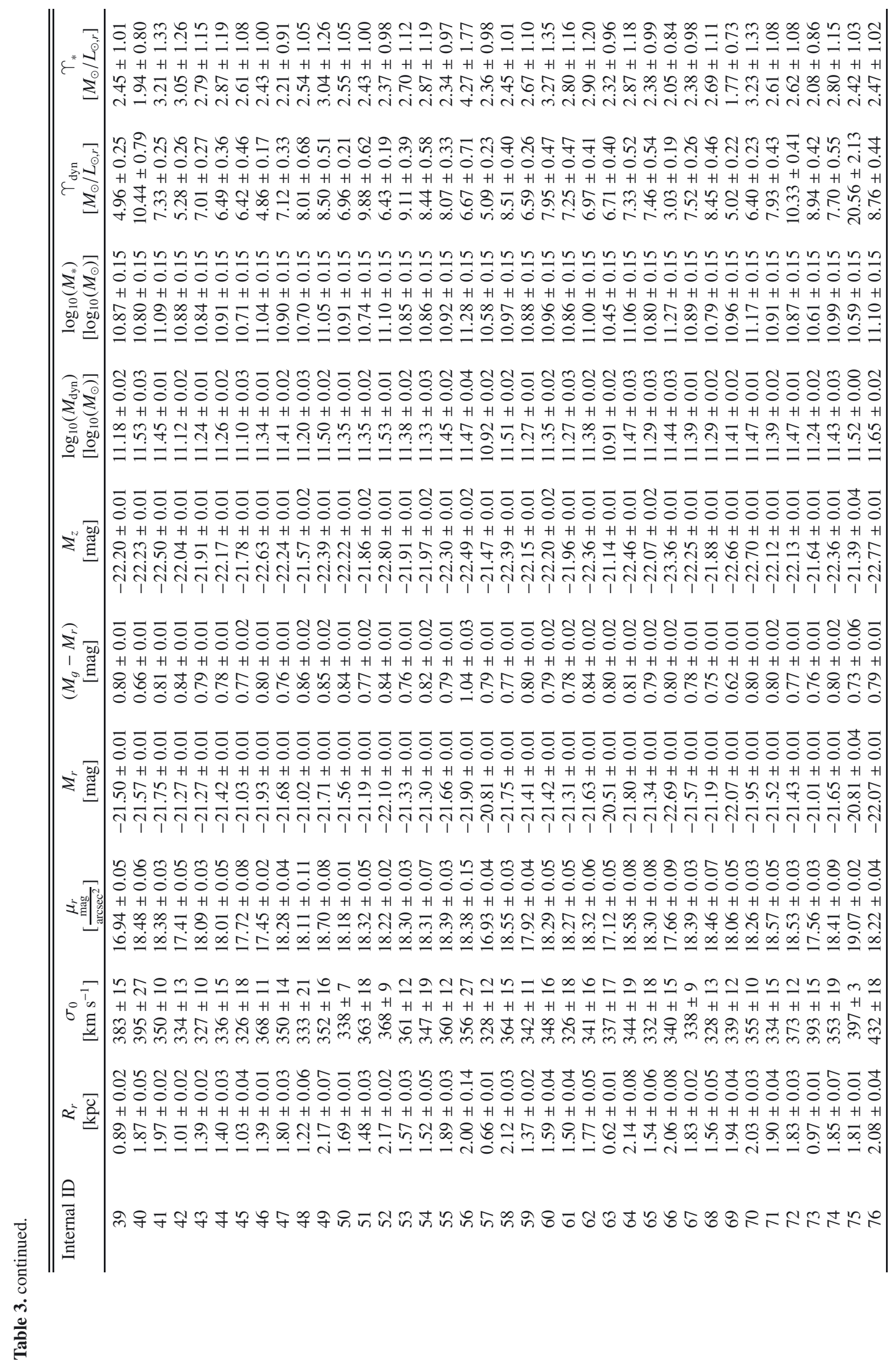




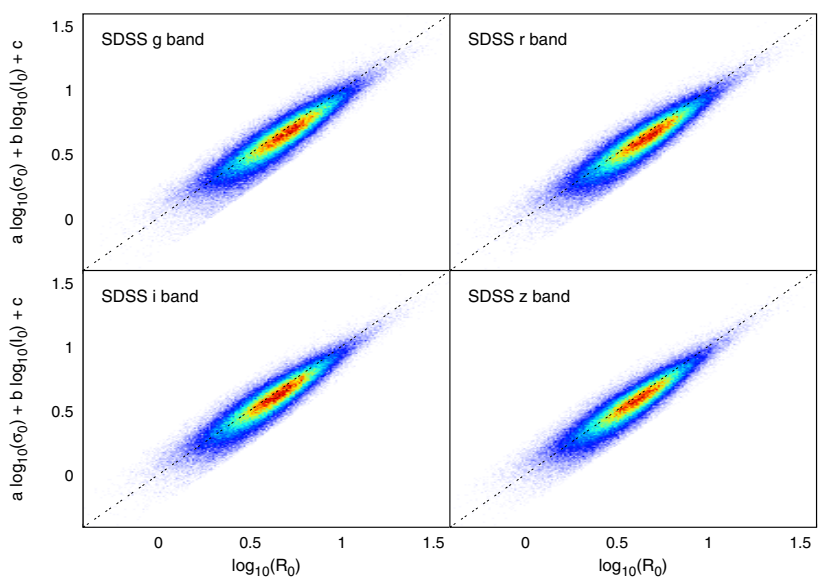

Fig. A.1. Edge-on projections of the fundamental plane of elliptical galaxies for four different SDSS filters using the de Vaucouleurs fit parameters. The $g$ band is shown in the top left panel and the $r$ band in the top right panel. The bottom left panel displays the $i$ band and the bottom right the $z$ band. The dashed black lines indicate the fundamental plane fits in the corresponding filters.

\section{Appendix A: Updated fundamental plane coefficients}

The fundamental plane, which was first mentioned in Terlevich et al. (1981) and properly defined and discussed Dressler et al. (1987) and Djorgovski \& Davis (1987), is an empirical relation between three global parameters of elliptical galaxies: the central velocity dispersion $\sigma_{0}$, the physical effective radius $R_{0}$, and the mean surface brightness $\mu_{0}$ within the effective radius. The last parameter is usually expressed as $I_{0}$, which is a renormalized surface brightness $\mu_{0}: \log _{10}\left(I_{0}\right)=-\frac{\mu_{0}}{2.5}$. The coefficients $a, b$, and $c$ are obtained by fitting

$\log _{10}\left(R_{0}\right)=a \cdot \log _{10}\left(\sigma_{0}\right)+b \cdot \log _{10}\left(I_{0}\right)+c$.

We provide updated values of the fundamental plane coefficients presented in Saulder et al. (2013). The main improvements are that we now use SDSS DR10 (Ahn et al. 2014) instead of SDSS DR8 (Aihara et al. 2011) and that we do not use any constraints on or information about the SDSS $u$ band, which we found to be quite problematic. Therefore, we have 133107 galaxies instead of 100427 for our basic sample (for definitions see Saulder et al. 2013), and after all filtering we end up with 119085 galaxies instead of the 92953 that are used for the final fit. This again makes it the largest sample ever used for calibrating the fundamental plane so far. In addition to improved fits, which are based on the de Vaucouleurs fit parameters directly from SDSS, we provide new fits for the $g$ and $r$ bands using the Sersic parameters from Simard et al. (2011). To this end, we use 121443 galaxies selected after some $3 \sigma$ clipping from the basic sample in this paper.

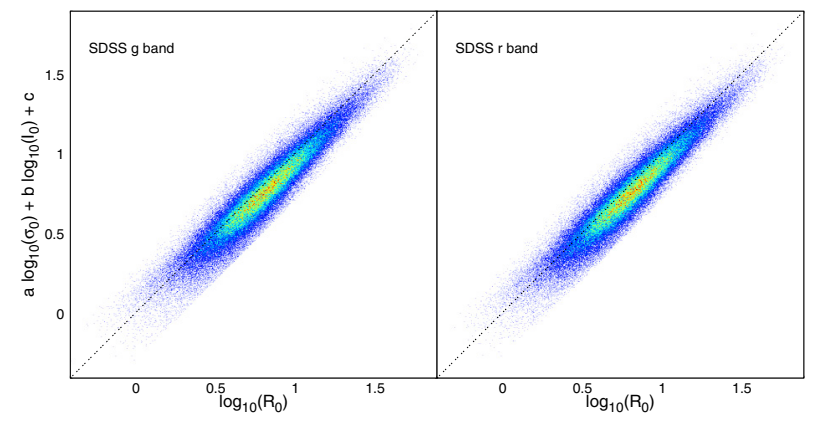

Fig. A.2. Edge-on projections of the fundamental plane of elliptical galaxies for the SDSS $g$ band (left panel) and $r$ band (right panel) using Sersic fit parameters. The dashed black lines indicate the fundamental plane fits in the corresponding filters.

Aside from the extended sample, there are a couple of other minor changes and improvements over the old paper (Saulder et al. 2013). First of all, we corrected a minor mistake in the calculation of the average distance error. This mistake caused the values of the error estimate in the old paper to be systematically lower by a couple of percentage points than they actually are. Even with the slightly larger error, it is still the best fit of the fundamental plane using a large sample at this wave-length range (Bernardi et al. 2003; Hyde \& Bernardi 2009; La Barbera et al. 2010).

Another improvement on the fit is that the volume weights are now considering that the sample only covers a limited redshift from 0.01 to 0.2 (or from 0.05 to 0.4 for the Sersic fits based on the basic sample in this paper). In our previous analysis, the very luminous galaxies were slightly under-represented, because their volume weights assumed a larger volume (the one in which they are theoretically still visible) than the volume of sample (redshift cut at 0.2). We then also subtract the volume corresponding to a redshift of 0.01 from the volume weights, where all galaxies were removed from the sample. The saturation limit of SDSS spectroscopy is also measured and included in the new volume weights by removing the volume associated with it in the same fashion as for the Malmquest bias limitation. The negligence of these two corrections caused the volume weights of very faint galaxies to be underestimated. Both corrections are relatively tiny, and the new coefficients are only slightly different from the old ones. In particular, the $a$ coefficient is moderately larger, hence closer to the values from the literature (see Table 1 in Saulder et al. 2013). The new coefficients are listed in Table A.1, edge-on projects of the fundamental plane for all four bands used for the de Vaucouleurs fit parameters in the calibration can be found in Fig. A.1, and the edge-on projects of the fundamental plane for the $g$ and $r$ SDSS bands using the Sersic fit parameters are displayed in Fig. A.2. 
Table A.1. Results of the best fits for the fundamental plane.

\begin{tabular}{c|ccccc}
\hline \hline Bands & $a$ & $b$ & $c$ & $s_{\varepsilon}$ & $\bar{\sigma}_{\text {dist }}[\%]$ \\
\hline$g(\mathrm{~d} V)$ & $0.999 \pm 0.026$ & $-0.754 \pm 0.011$ & $-7.93 \pm 0.10$ & 0.0942 & 19.3 \\
$r(\mathrm{~d} V)$ & $1.070 \pm 0.026$ & $-0.770 \pm 0.011$ & $-7.98 \pm 0.11$ & 0.0935 & 19.0 \\
$i(\mathrm{~d} V)$ & $1.100 \pm 0.026$ & $-0.775 \pm 0.012$ & $-7.96 \pm 0.11$ & 0.0919 & 18.6 \\
$z(\mathrm{~d} V)$ & $1.145 \pm 0.025$ & $-0.781 \pm 0.012$ & $-8.02 \pm 0.11$ & 0.0920 & 18.5 \\
\hline$g(\mathrm{~S})$ & $0.966 \pm 0.026$ & $-0.726 \pm 0.009$ & $-7.62 \pm 0.09$ & 0.0977 & 20.6 \\
$r(\mathrm{~S})$ & $1.029 \pm 0.026$ & $-0.729 \pm 0.009$ & $-7.56 \pm 0.09$ & 0.0972 & 20.4 \\
\hline
\end{tabular}

Notes. The coefficients $a, b$, and $c$ for 4 SDSS bands using redshift evolution, volume weights, $3 \sigma$ clipping, and the radii and magnitudes of de Vaucouleurs $(\mathrm{d} V)$ fits are provided in this table. Furthermore, it contains the coefficients for 2 SDSS bands using the same calibration, but the radii and magnitudes from the Sersic (S) fits of Simard et al. (2011). The root mean square $s_{\varepsilon}$ of the fits and the relative distance error $\bar{\sigma}_{\text {dist }}$ of the fundamental plane are also provided.

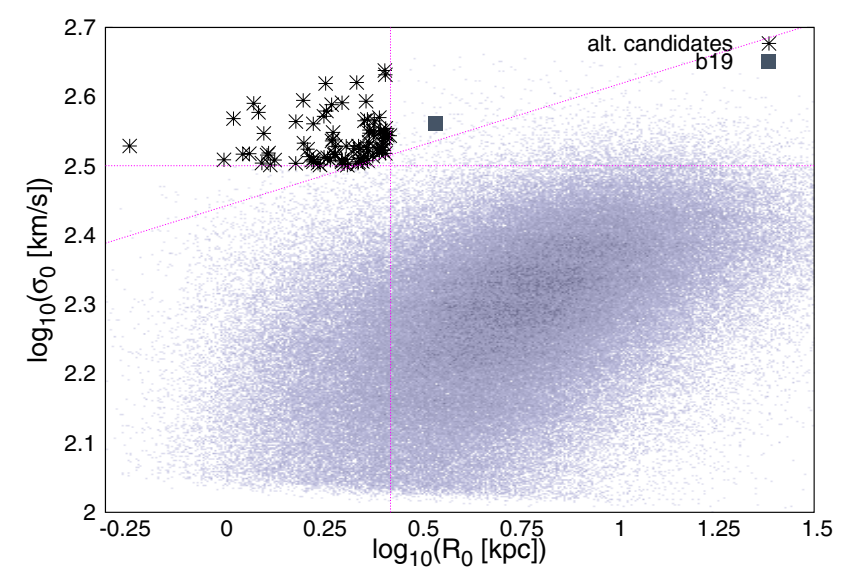

Fig. B.1. Selection of the alternative candidates in the $R_{0} \sigma_{0}$ plane. The restrictions, which define our alternative candidates, are indicated by the dashed magenta lines. The black stars represent the 85 candidates for galaxies with similar properties in Sersic fit parameters as b19, and b19 itself is represented by a grey filled square in the plot.

\section{Appendix B: The Sersic fit sample}

In addition to the candidate sample defined using the de Vaucouleurs fit parameters, we provide an alternative sample using the Sersic fit parameters from Simard et al. (2011).

The sample is defined in the same fashion as the main candidate sample, and we find 85 galaxies fulfilling the requirements (listed in Table B.1, together with b19, which was assigned the Sersic ID 1). The logarithm of the physical radius $R_{0}$ has to be smaller than the sample's average by at least one standard deviation, which provides us with an upper limit for $R_{0}$ of $\sim 2.65 \mathrm{kpc}$. The lower limit for the central velocity dispersion $\sigma_{0}$ of $\sim 316.6 \mathrm{~km} \mathrm{~s}^{-1}$ is obtained by requiring it to be at least two standard deviations higher than the mean of the logarithm of the central velocity dispersion. The last criterion ensures that all candidates are more than three standard deviations off from the $\log _{10}\left(R_{0}\right)-\log _{10}\left(\sigma_{0}\right)$ relation, which was obtained by a linear fit to the data points. The selection criteria is illustrated in

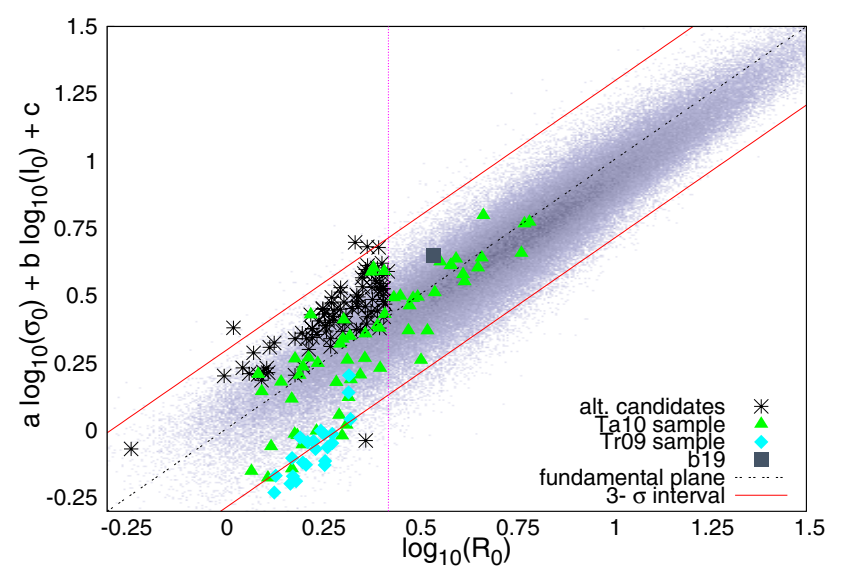

Fig. B.2. Location of the candidate galaxies on the fundamental plane using Sersic fit parameters. The candidates are indicated by black stars. The galaxies belonging to the Ta10 sample are represented using filled green triangles, and the Tr09 sample is marked by filled cyan diamonds. The starting point of our investigation, b19, is indicated by a filled grey square. The magenta dotted lines show the limiting physical radius used in the sample sample selection. The black dashed lines are the fundamental plane fits from Appendix A with their corresponding $3 \sigma$ confidence intervals shown as red solid lines. The fit appears to be slightly offset owing to the volume weights used to correct the Malmquist bias in the fitting process.

Fig. B.1. We find that b19 fails to fulfil the radial size requirement in the case of the Sersic fit parameters (see Table B.2 for numbers), and it is not included in the 85 alternative candidates. However, we keep on providing its position in the plots and tables. As illustrated in Figs. B.2 to B.9, the alternative sample has generally speaking similar properties to the main candidate sample, but it is less cohesive and more scattered. We therefore prefer our main sample to this one. There are 51 galaxies, which the two candidate lists have in common (see Table C.1). We consider these galaxies as candidates with increased priority for any follow-up observations. 


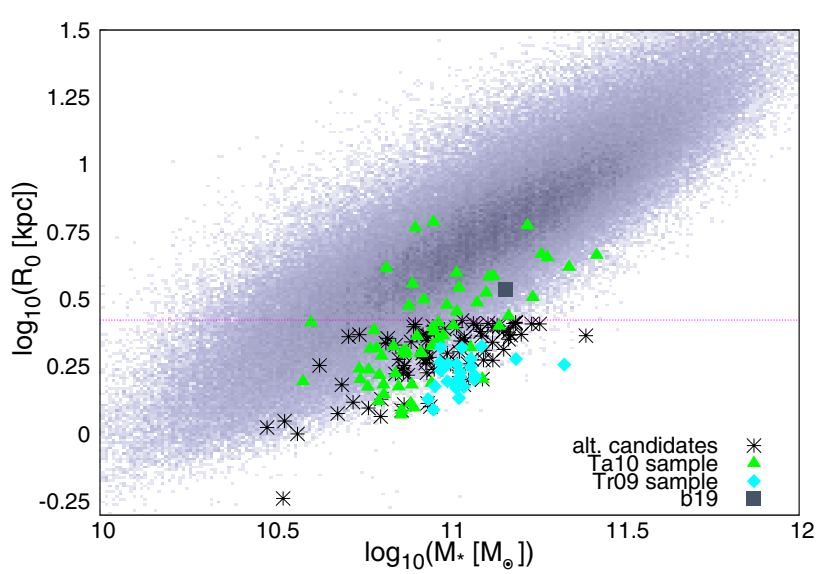

Fig. B.3. Stellar mass-size relation for our basic sample using the Sersic fit parameters. The alternative candidates are indicated by black stars. The galaxies belonging to the Ta10 sample are represented using filled green triangles, and the Tr09 sample is indicated by filled cyan diamonds. B19 is indicated by a filled grey square. The magenta dashed line denotes the limiting scaling radius for our sample selection.

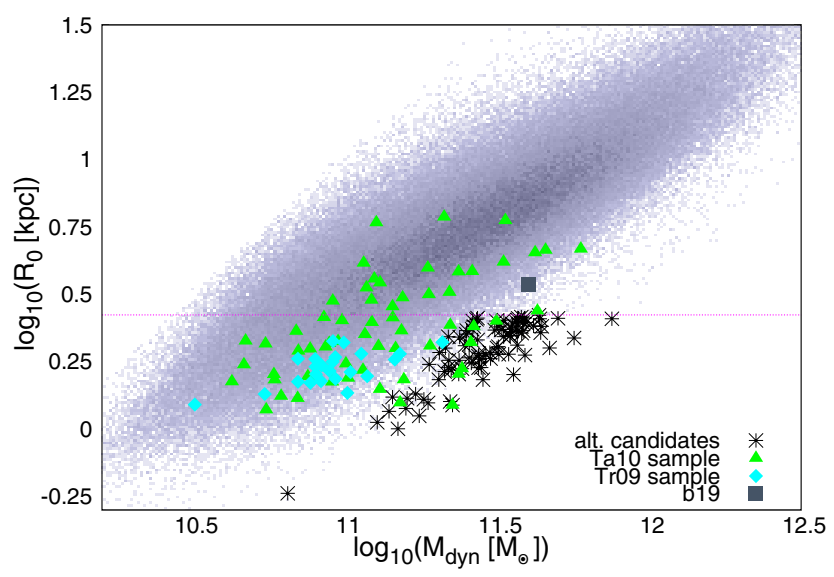

Fig. B.4. Dynamical mass-size relation for our basic sample using the Sersic fit parameters. The alternative candidates are indicated by black stars. The galaxies belonging to the Ta10 sample are represented using filled green triangles, and the Tr09 sample is indicated by filled cyan diamonds. B19 is indicated by a filled grey square. The magenta dashed line denotes the limiting scaling radius for our sample selection.

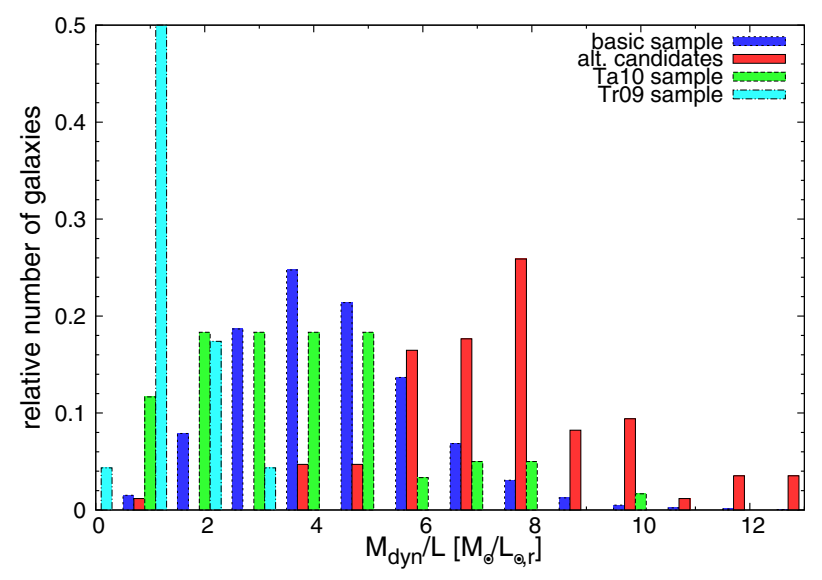

Fig. B.5. Distribution of the dynamical mass-to-light ratios $\Upsilon_{\text {dyn }}$ using the Sersic fit parameters. The blue histogram corresponds to our basic sample, which only consists of early-type galaxies. The green histogram represents the Ta10 sample, while the cyan histogram corresponds to Tr09 sample. The red histogram denotes our 85 alternative candidates.

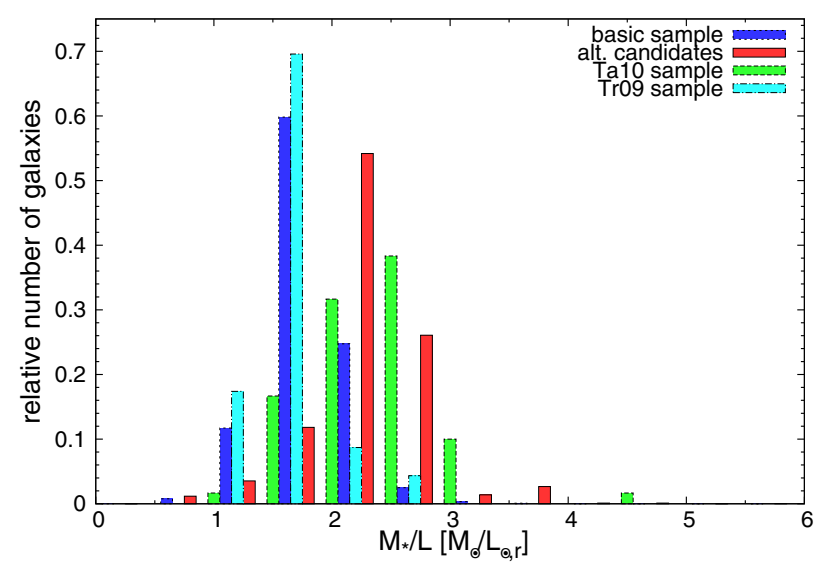

Fig. B.6. Distribution of the stellar mass-to-light ratios $\Upsilon_{*}$ using the Sersic fit parameters. The blue histogram corresponds to our basic sample, which only consists of early-type galaxies. The green histogram represents the Ta10 sample, while the cyan histogram corresponds to Tr09 sample. The red histogram denotes our 85 alternative candidates. 


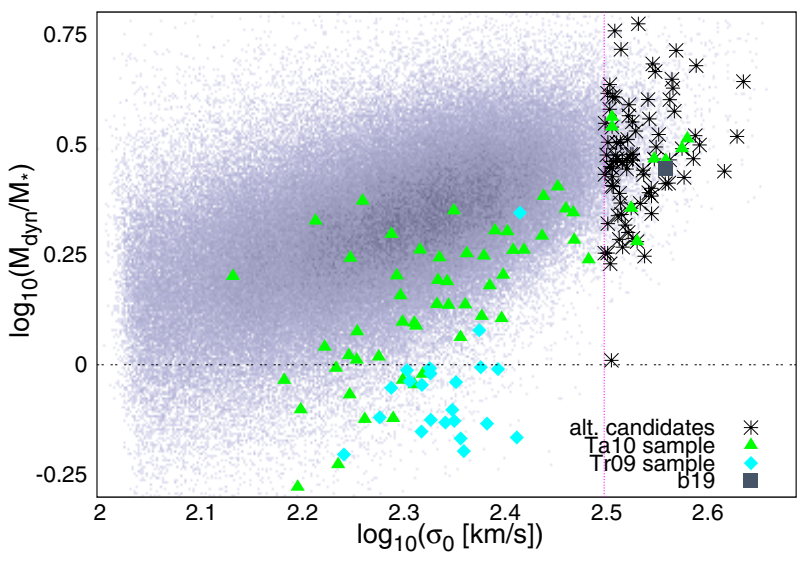

Fig. B.7. Dependence of the dynamical mass $M_{\mathrm{dyn}}$ to stellar mass $M_{*}$ ratio on central velocity dispersion $\sigma_{0}$ using the Sersic fit parameters. The alternative candidates are indicated by black stars. The galaxies belonging to the Ta10 sample are represented using filled green triangles and the Tr09 sample is marked by filled cyan diamonds. B19 is indicated by a filled grey square. The magenta dashed line marks the limiting scaling central velocity dispersion for our sample selection. The area below the black dashed line is considered to be unphysical, because $M_{*}$ would exceed $M_{\text {dyn }}$.

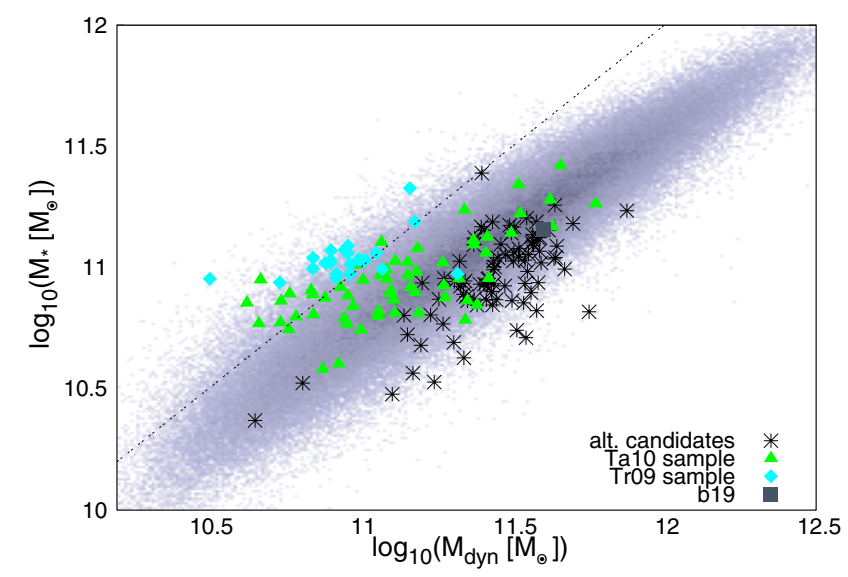

Fig. B.8. Distribution of the sample's galaxies in the dynamical mass $M_{\text {dyn }}$ vs. stellar mass $M_{*}$ plane using the Sersic fit parameters. The alternative candidates are indicated by black stars. The galaxies belonging to the Ta10 sample are represented using filled green triangles, and the Tr09 sample is indicated by filled cyan diamonds. B19 is indicated by a filled grey square.The magenta dashed line marks the limiting scaling central velocity dispersion for our sample selection. The black dashed line denotes the limit of the $M_{\mathrm{dyn}}$ to $M_{*}$ ratio, which is still considered to be physical, because $M_{*}$ would exceed $M_{\text {dyn }}$ above it.

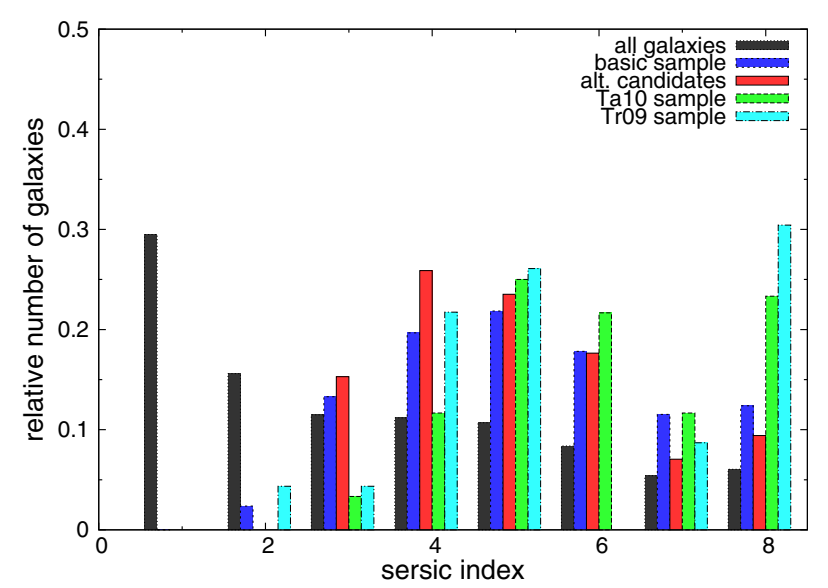

Fig. B.9. Distribution of the Sersic indices of different samples of galaxies. The black histogram of the Sersic indices stands for all galaxies in SDSS DR7 for which Simard et al. (2011) did their refits. The blue histogram indicates the distribution of Sersic parameters for our basic sample, which only consists of early-type galaxies. The green histogram represents the Ta10 sample, while the cyan histogram corresponds to Tr09 sample. The red histogram denotes our 85 alternative candidates. 
C. Saulder et al.: Dozens of compact and high velocity-dispersion, early-type galaxies in Sloan Digital Sky Survey

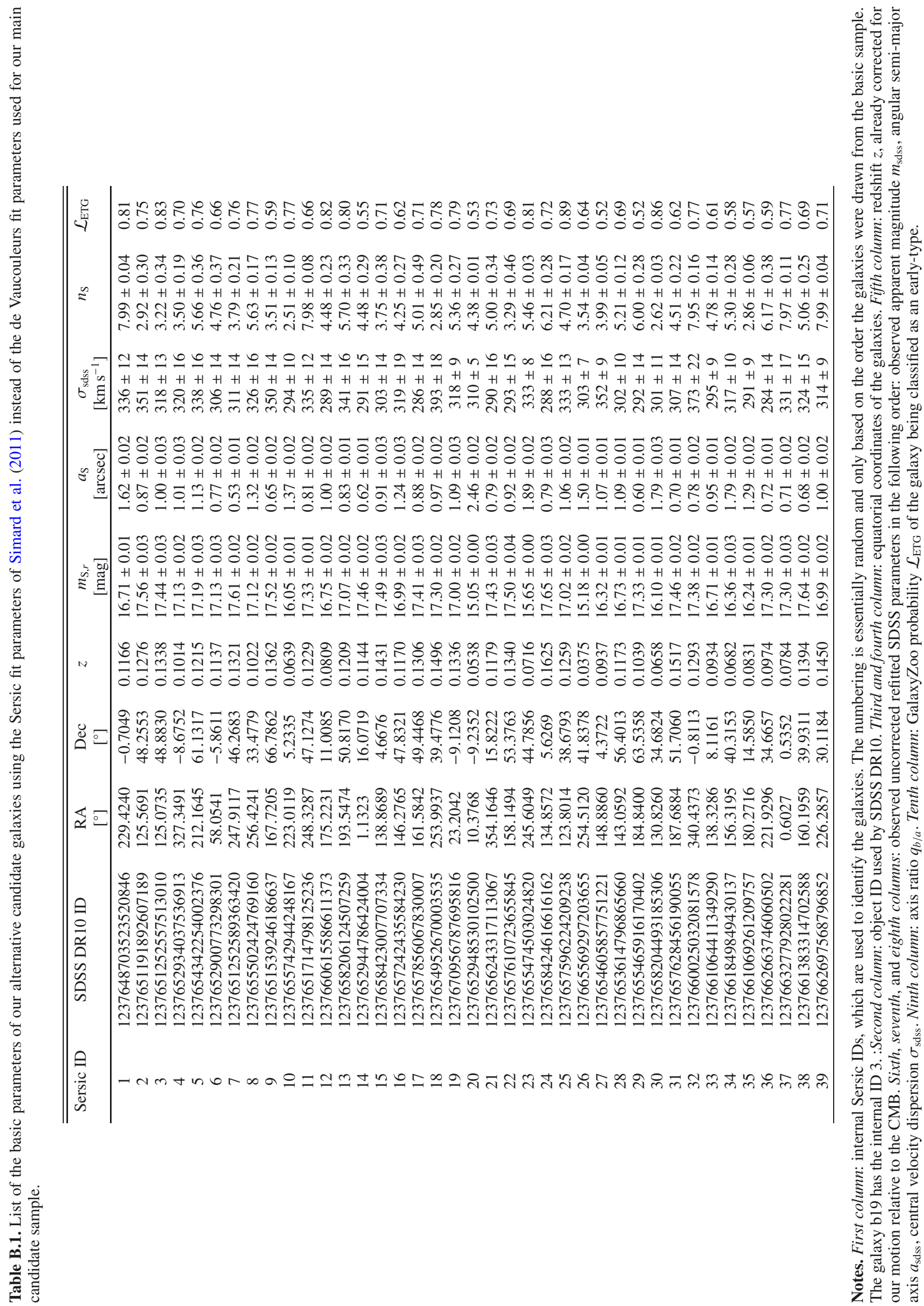




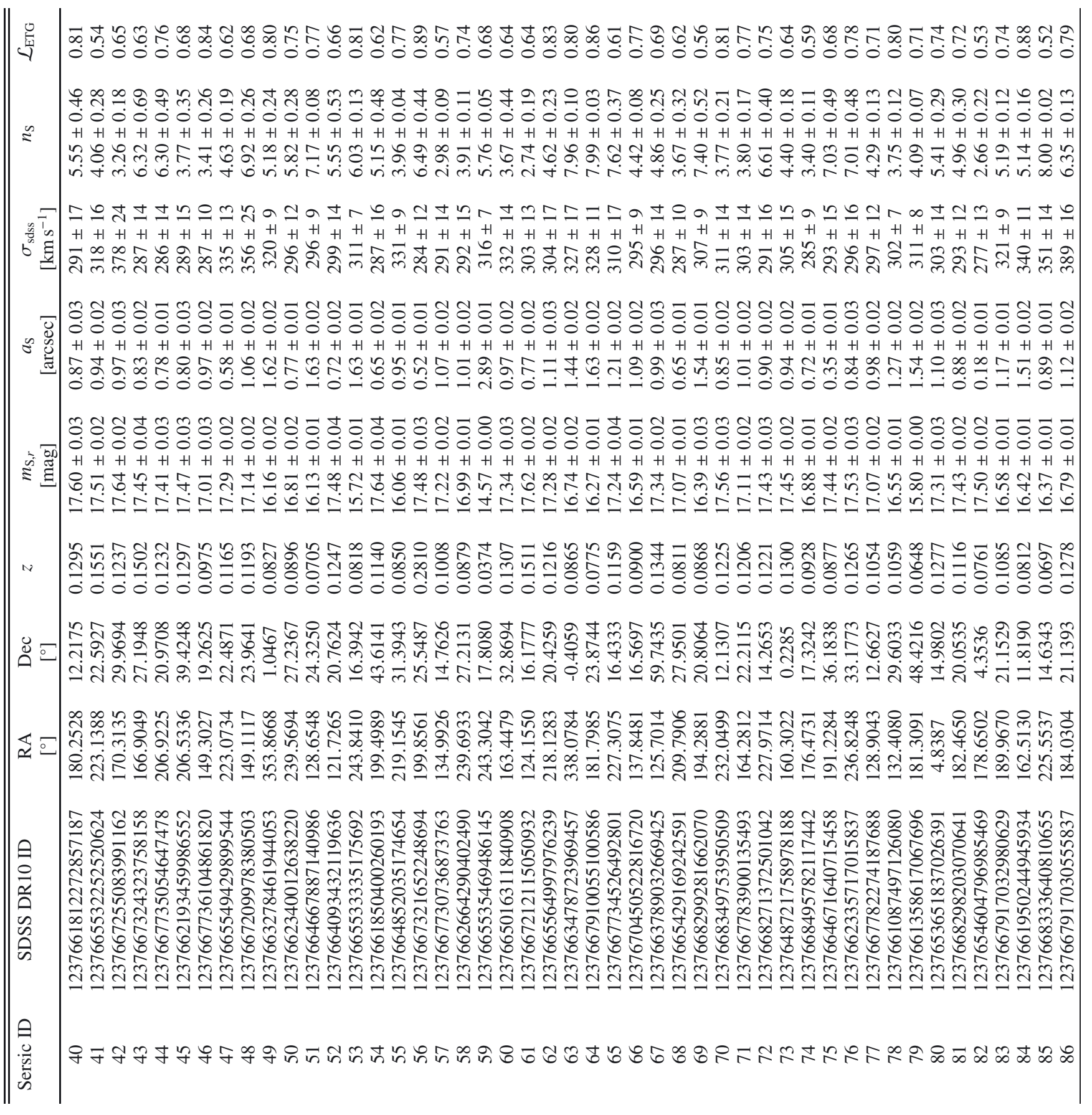




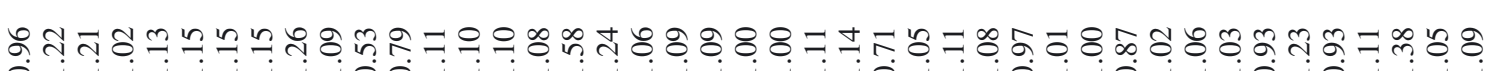

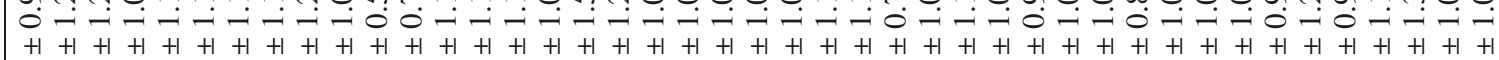

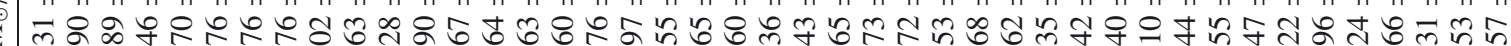

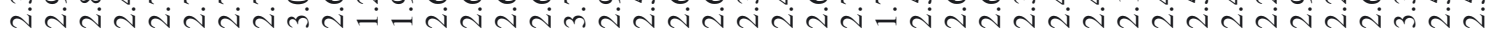

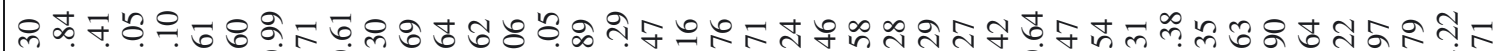
年

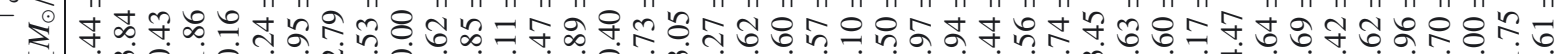

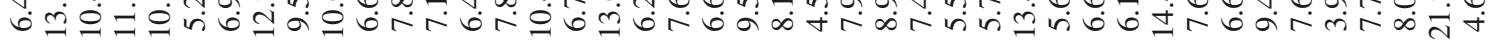

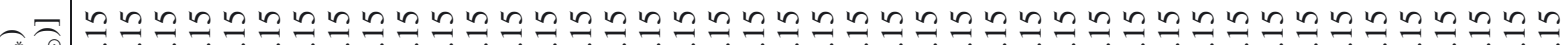

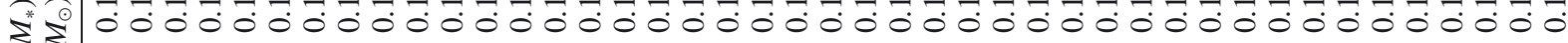
- $1+1+1+1+1+1+1+1+1+1+1+1+1+1+1+1+1+1+1+1+1+1+1+1+1+1+1+1+1+1+1+1+1+1+1+1+1+1+1+1+1+1+1$

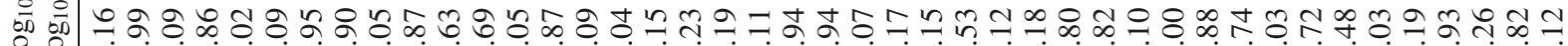

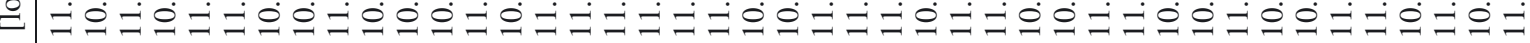

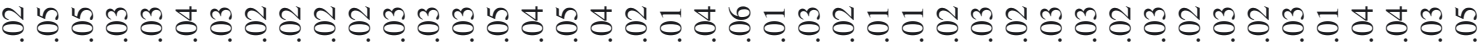
-

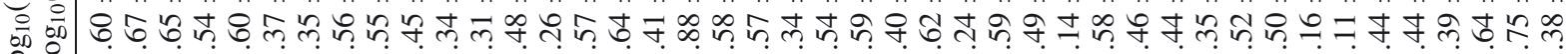

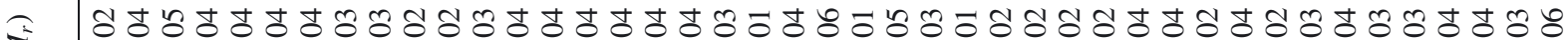

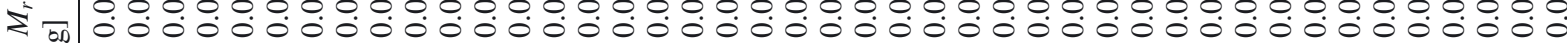
$1 \Xi 5+1+1+1+1+1+1+1+1+1+1+1+1+1+1+1+1+1+1+1+1+1+1+1+1+1+1+1+1+1+1+1+1+1+1+1+1+1+1+1+1+1+1+1$ క 0000000000000000000000000000000000000000000000000000

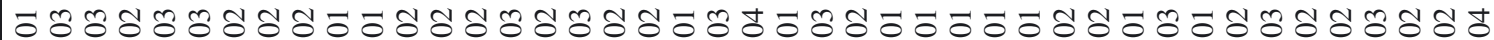
0000000000000000000000000000000000000000000 $\begin{array}{lll} & \\ 1 & 0\end{array}$

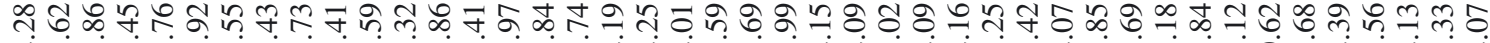

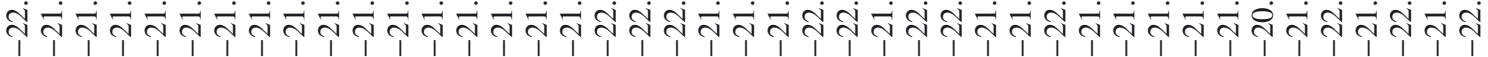

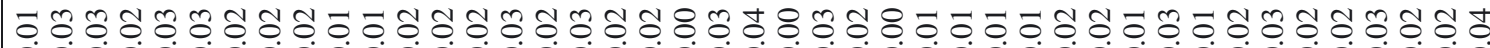

$\leq$ 盟

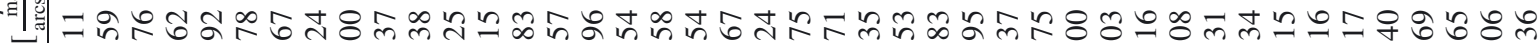

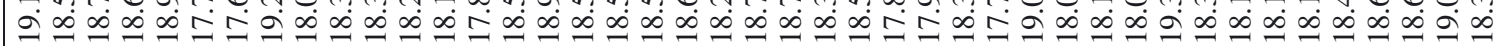

F

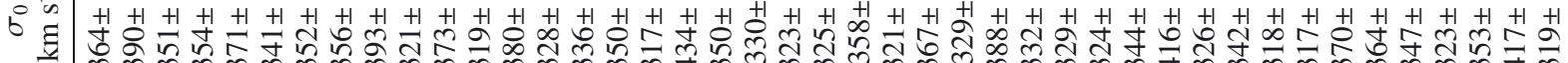

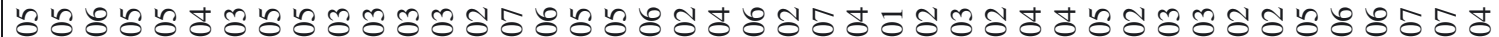

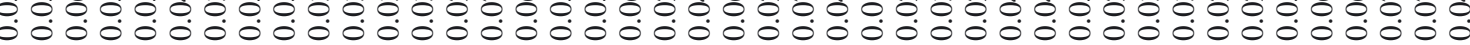

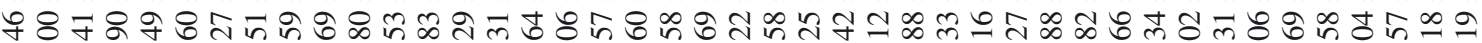

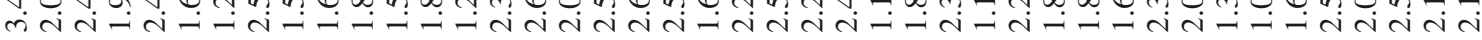

急

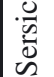




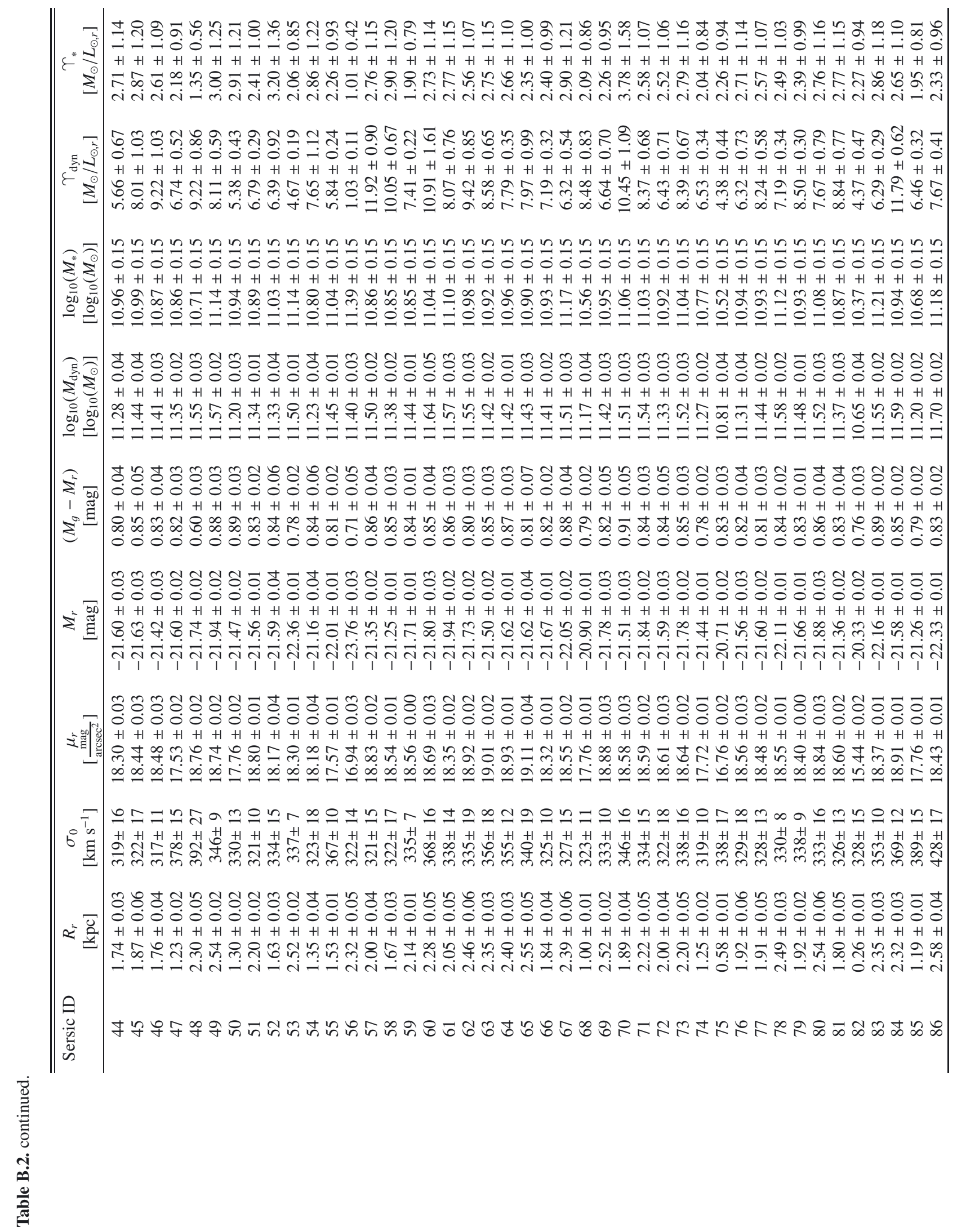


C. Saulder et al.: Dozens of compact and high velocity-dispersion, early-type galaxies in Sloan Digital Sky Survey

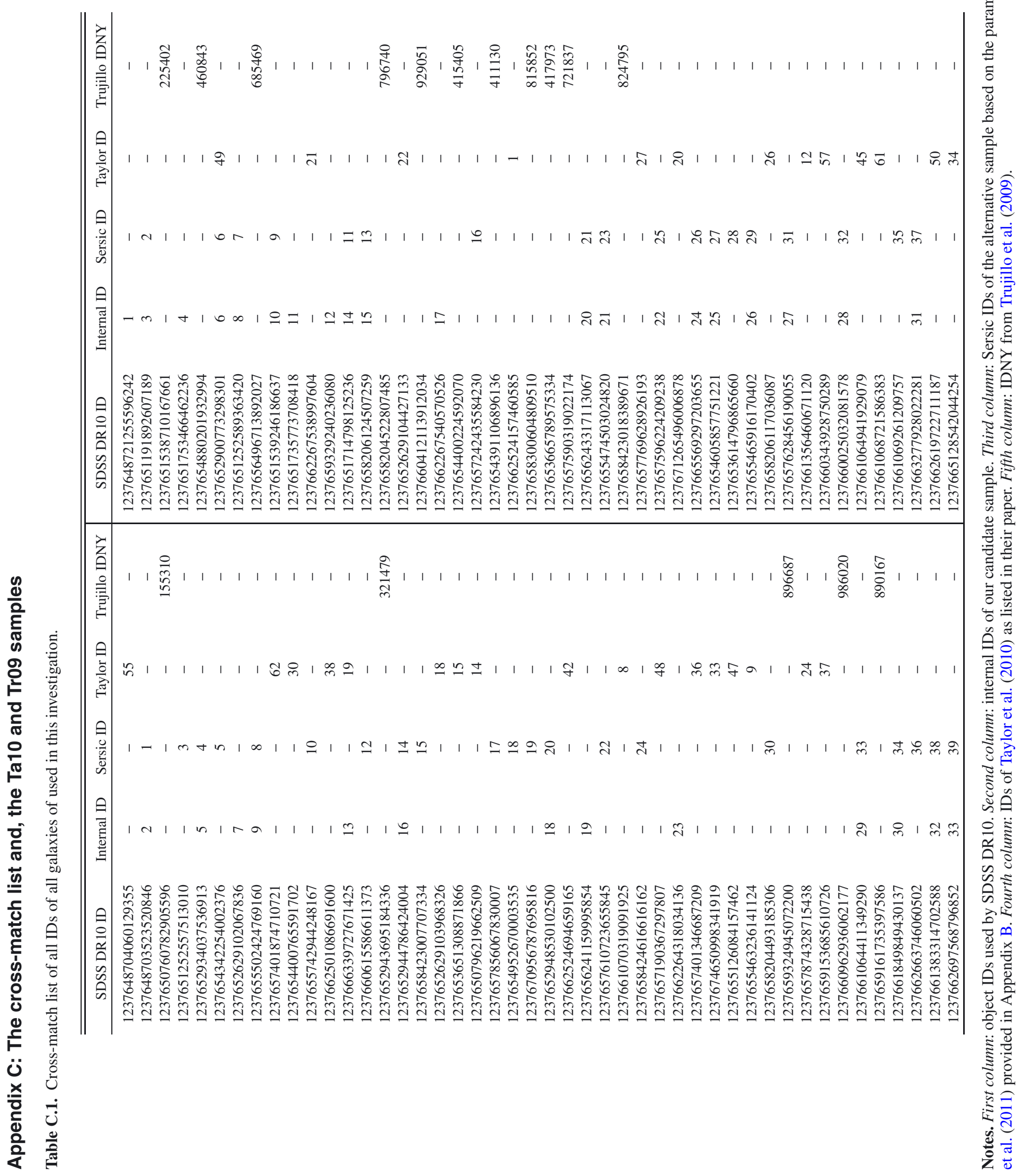




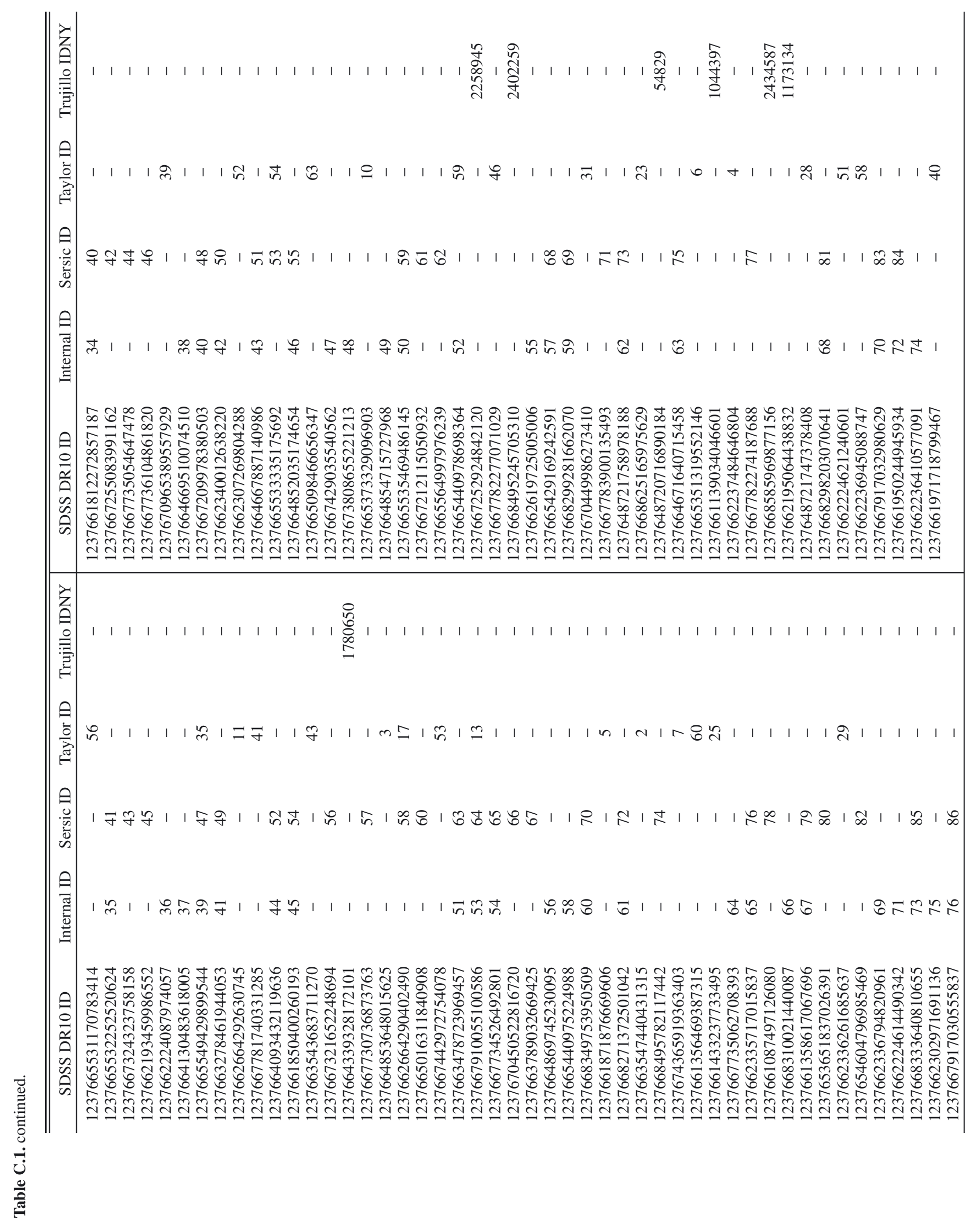


C. Saulder et al.: Dozens of compact and high velocity-dispersion, early-type galaxies in Sloan Digital Sky Survey

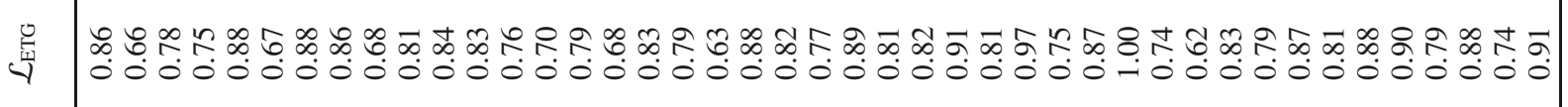
๖ † 00 00000000000000000000000000000000000000000

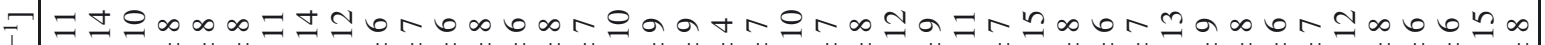

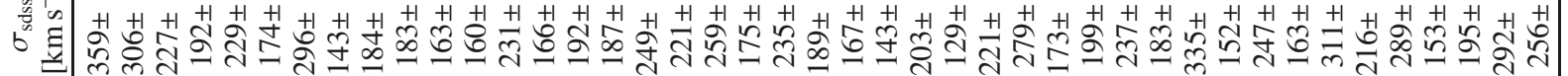

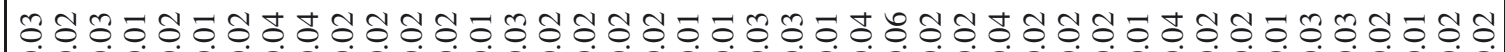
n 0000000000000000000000000000000000000000000

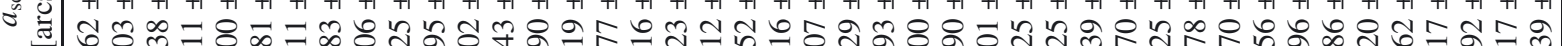

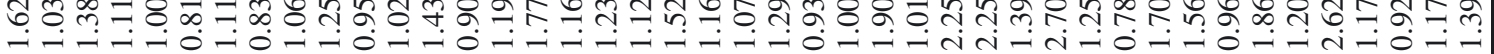

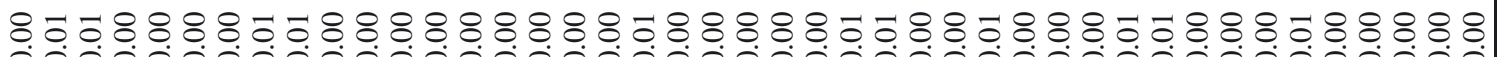
000

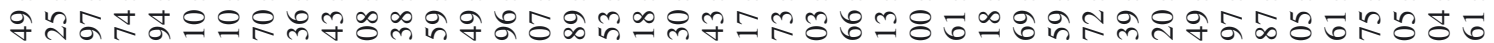

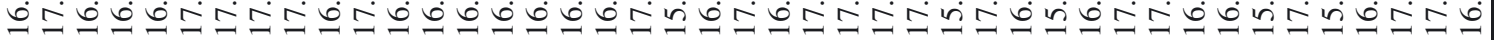

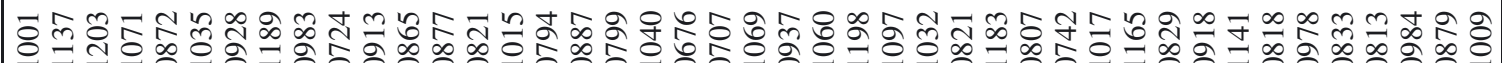

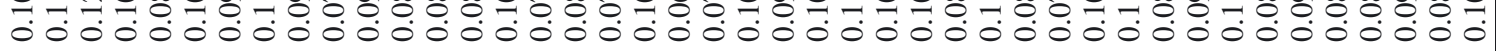

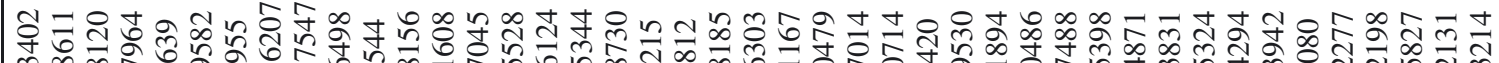
过

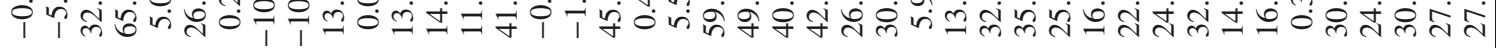

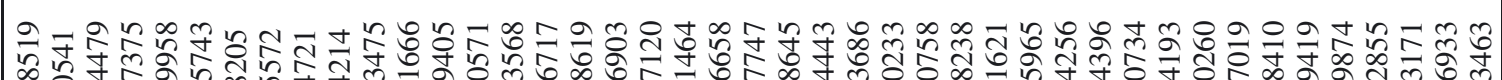
๔ర तิ

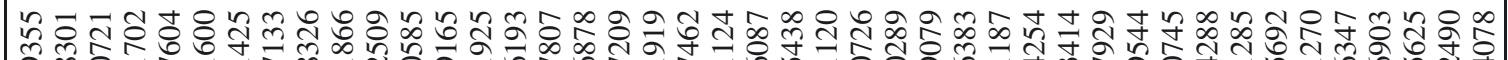

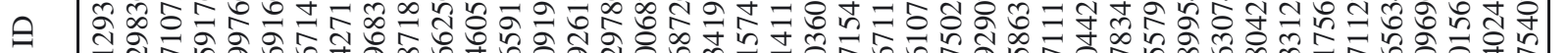

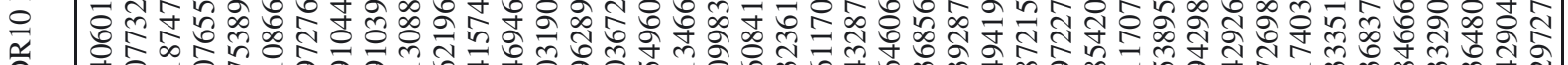

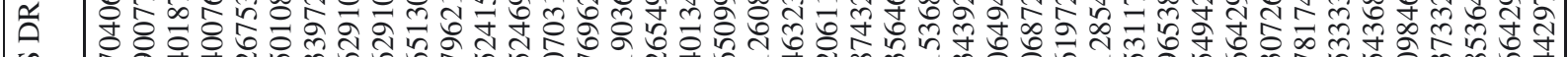

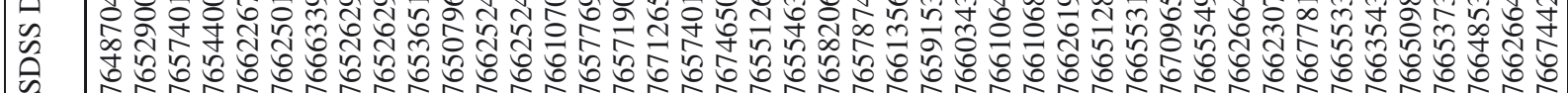

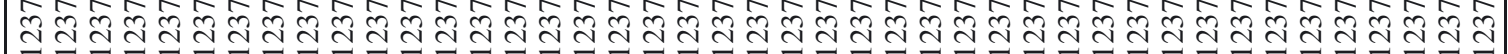

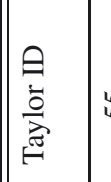

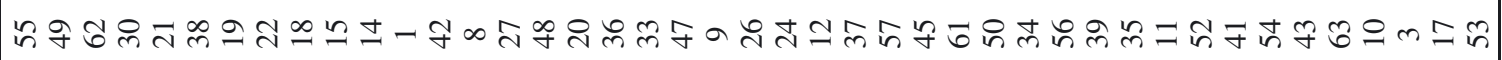




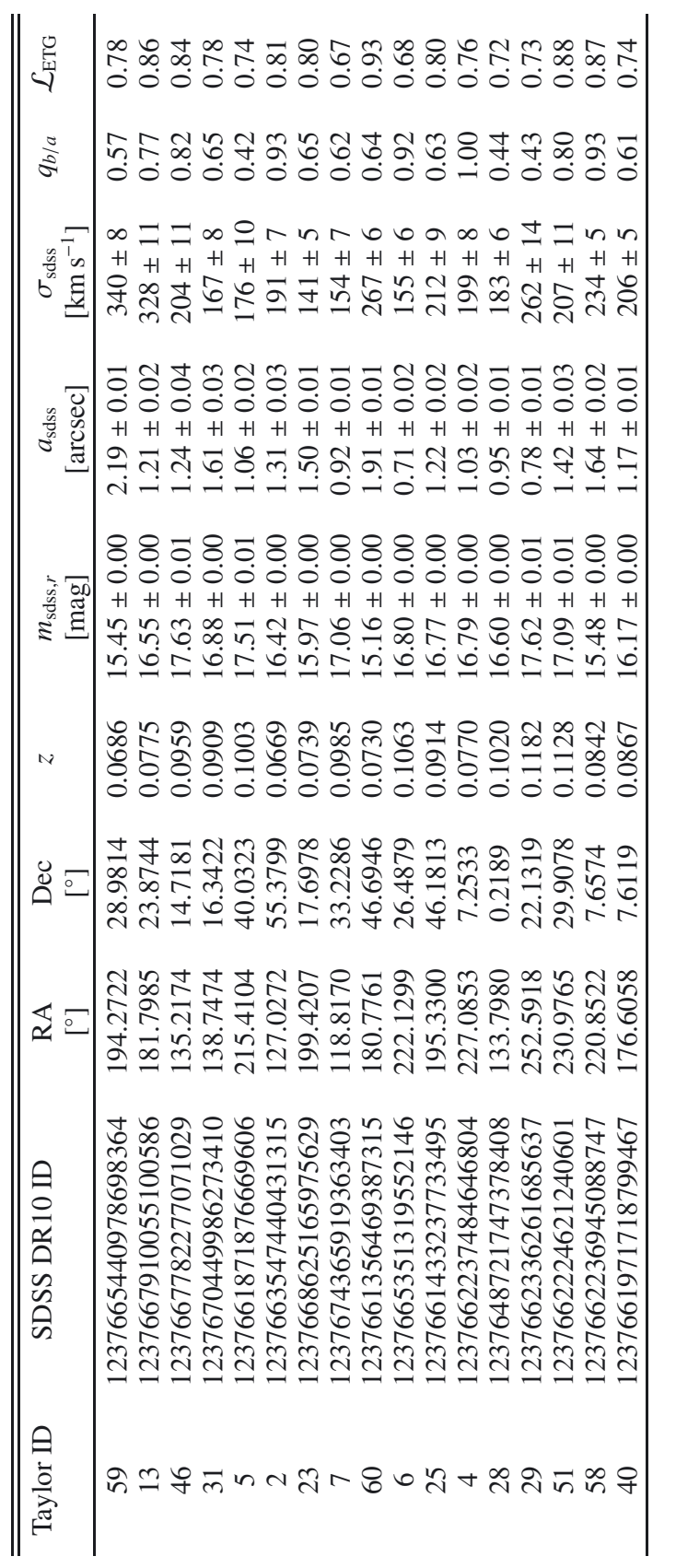


$-0-0-10-1-10-0000-000000-0-00-00-0-0 .-1-10-1$ $+1+1+1+1+1+1+1+1+1+1+1+1+1+1+1+1+1+1+1+1+1+1+1+1+1+1+1+1+1+1+1+1+1+1+1+1+1+1+1+1+1+1+1$ กำ

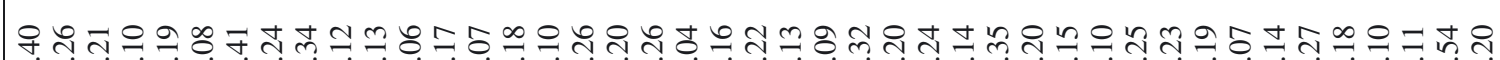
否

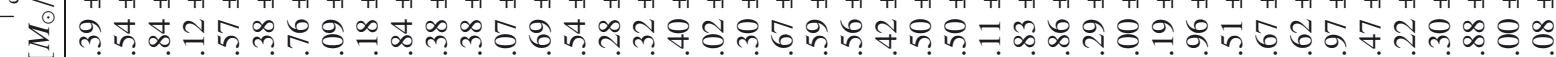
बें

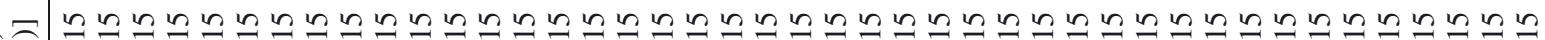
\& $\$ 000000000000000000000000000000000000000000$ $2+1+1+1+1+1+1+1+1+1+1+1+1+1+1+1+1+1+1+1+1+1+1+1+1+1+1+1+1+1+1+1+1+1+1+1+1+1+1+1+1+1+1+1$

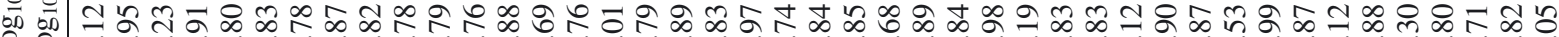

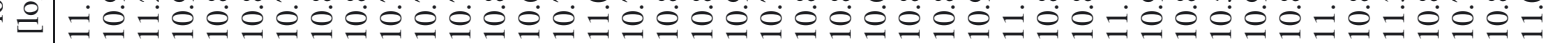

= $25+1+1+1+1+1+1+1+1+1+1+1+1+1+1+1+1+1+1+1+1+1+1+1+1+1+1+1+1+1+1+1+1+1+1+1+1+1+1+1+1+1+1$ 高

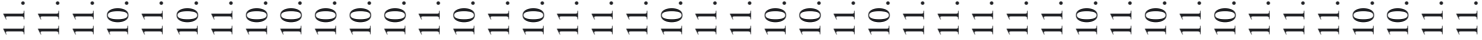

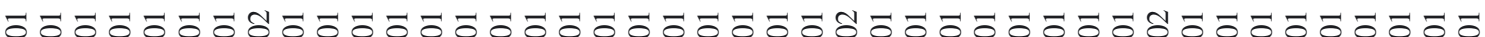
00000000000000000000000000000000000000 $+1+1+1+1+1+1+1+1+1+1+1+1+1+1+1+1+1+1+1+1+1+1+1+1+1+1+1+1+1+1+1+1+1+1+1+1+1+1+1+1+1+1+1$

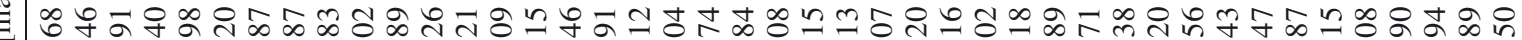

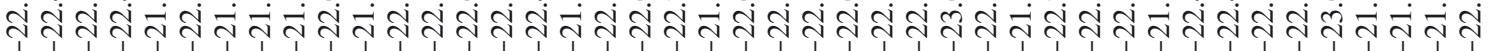

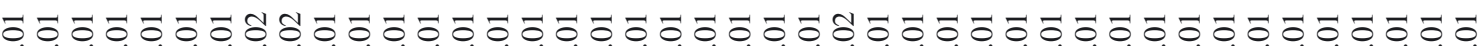

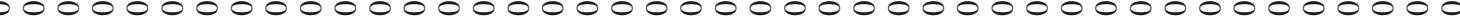
1
$H+1$

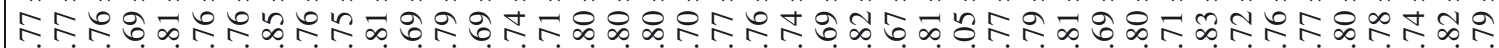
0.00000000000000000000000000

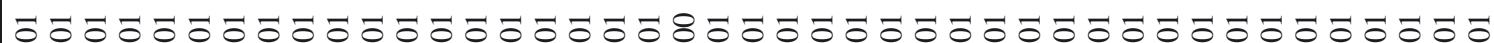

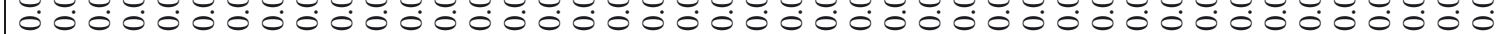

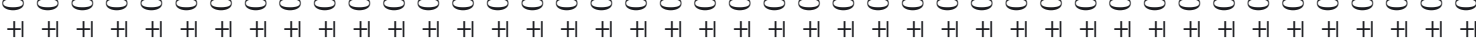
和范

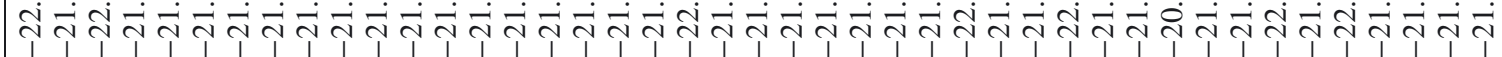

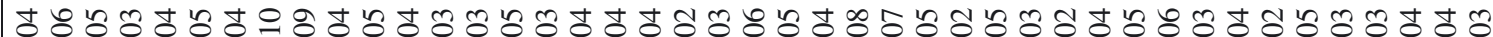

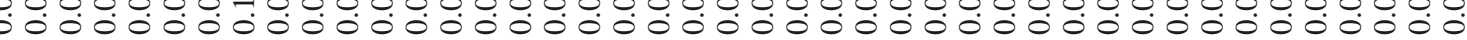
$\infty$

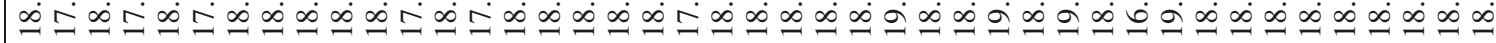

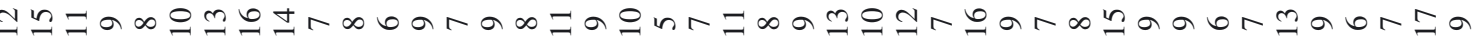

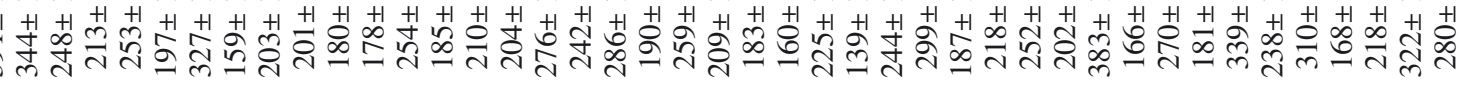

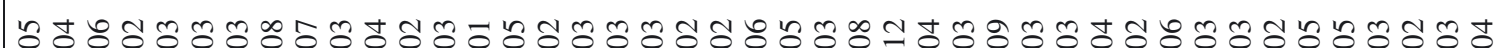

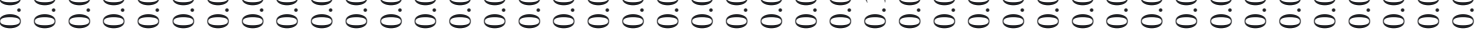
$+1+1+1+1+1+1+1+1+1+1+1+1+1+1+1+1+1+1+1+1+1+1+1+1+1+1+1+1+1+1+1+1+1+1+1+1+1+1+1+1+1$

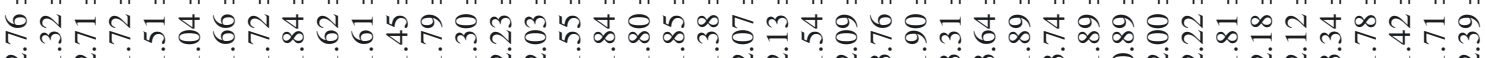




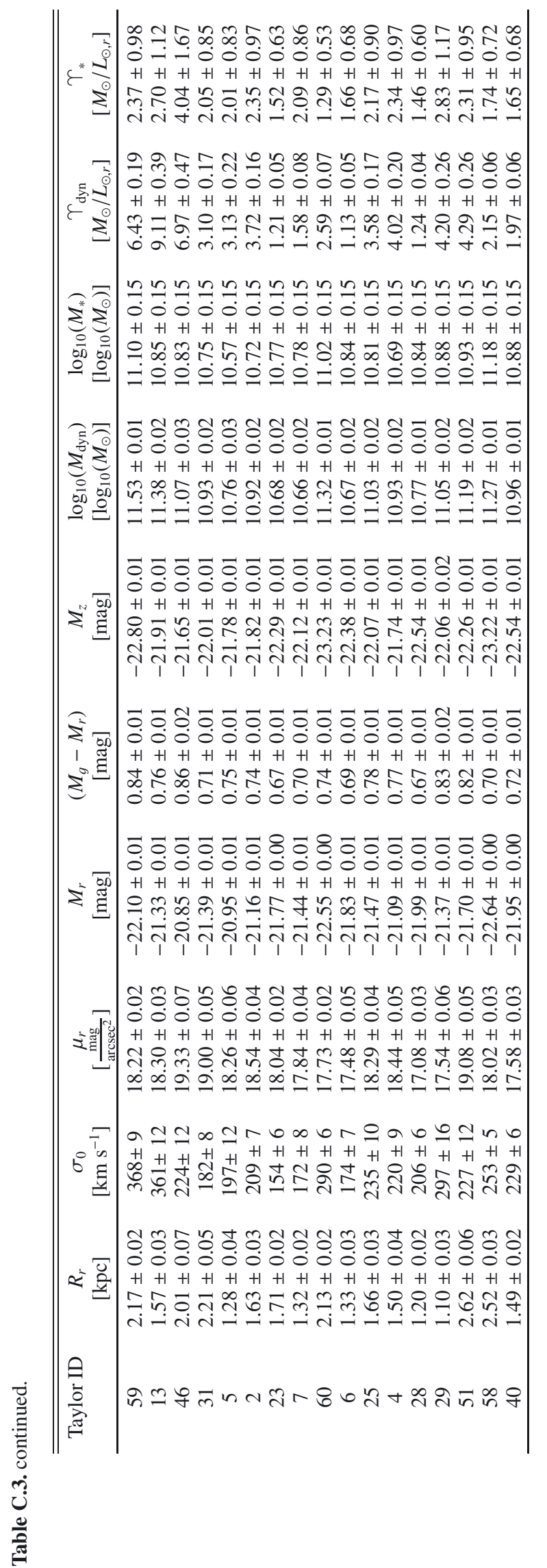




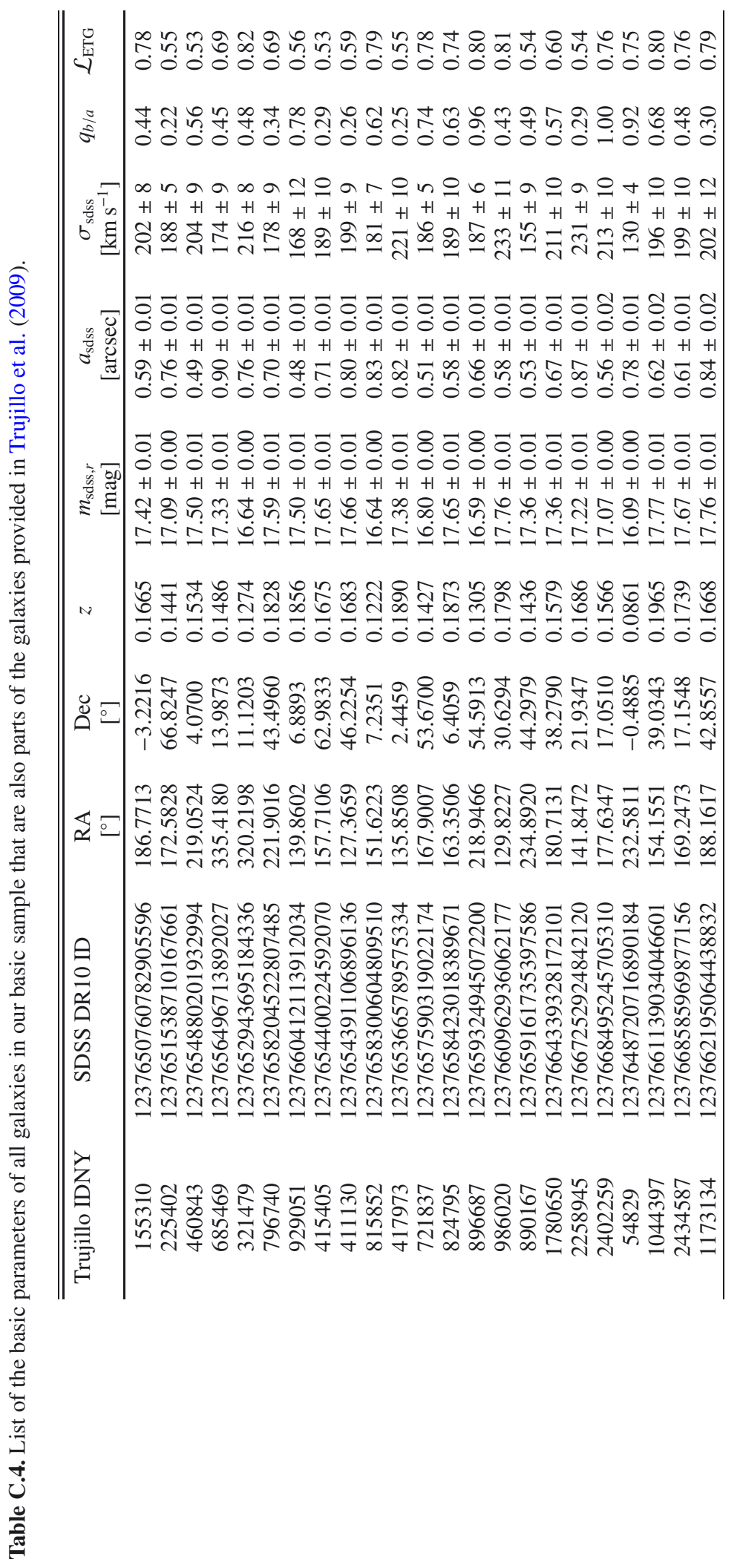




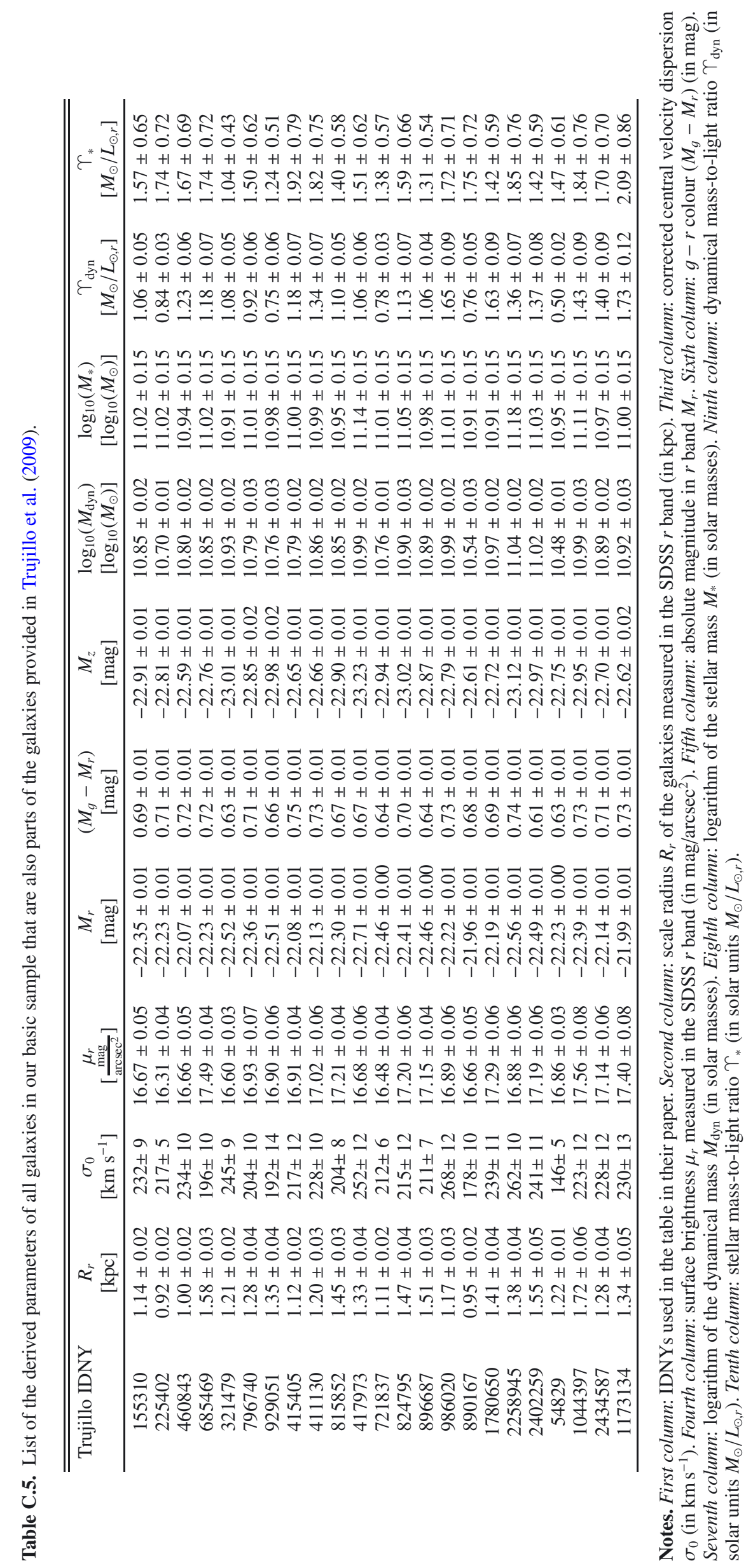

